HD-THEP-96-38

\title{
High Energy Collisions and Nonperturbative QCD马
}

\author{
O. Nachtmann \\ Institut für Theoretische Physik, Universität Heidelberg, \\ Philosophenweg 16, D-69120 Heidelberg, FRG
}

\begin{abstract}
We discuss various ideas on the nonperturbative vacuum structure in QCD. The stochastic vacuum model of Dosch and Simonov is presented in some detail. We show how this model produces confinement. The model incorporates the idea of the QCD vacuum acting like a dual superconductor due to an effective chromomagnetic monopole condensate. We turn then to high energy, small momentum transfer hadron-hadron scattering. A field-theoretic formalism to treat these reactions is developed, where the basic quantities governing the scattering amplitudes are correlation functions of light-like Wegner-Wilson lines and loops. The evaluation of these correlation functions with the help of the Minkowskian version of the stochastic vacuum model is discussed. A further surprising manifestation of the nontrivial vacuum structure in QCD may be the production of anomalous soft photons in hadron-hadron collisions. We interpret these photons as being due to "synchrotron radiation from the vacuum". A duality argument leads us from there to the expectation of anomalous pieces proportional to $\left(Q^{2}\right)^{1 / 6}$ in the electric form factors of the nucleons for small $Q^{2}$. Finally we sketch the idea that in the Drell-Yan reaction, where a quark-antiquark pair annihilates with the production of a lepton pair, a "chromodynamic Sokolov-Ternov effect" may be at work. This leads to a spin correlation of the $q \bar{q}$ pair, observable through the angular distribution of the lepton pair.
\end{abstract}

\footnotetext{
${ }^{1}$ Based on lectures given at the workshop "Topics in Field Theory", organized by the Graduiertenkolleg Erlangen-Regensburg 1993 in Banz, Germany, and at the "35. Internationale Universitätswochen für Kern- und Teilchenphysik 1996" in Schladming, Austria.
} 


\section{Table of Contents}

1. Introduction

2. The QCD Vacuum

2.1 Connectors

2.2 The non-abelian Stokes theorem

2.3 The cumulant expansion

2.4 The basic assumptions of the stochastic vacuum model

2.5 The Wegner-Wilson loop in the stochastic vacuum model

3. Soft Hadronic Reactions

3.1 General considerations

3.2 The functional integral approach to parton-parton scattering

3.3 The Eikonal expansion

3.4 The quark-quark scattering amplitude

3.5 The scattering of systems of quarks, antiquarks, and gluons

3.6 The scattering of wave packets of partons representing mesons

3.7 The evaluation of scattering amplitudes in the Minkowskian version of the stochastic vacuum model

4. "Synchrotron Radiation" from the vacuum, electromagnetic form factors of hadrons, and spin correlations in the Drell-Yan reaction

5. Conclusions

Appendices

A. Higher cumulant terms and dynamic fermions in the calculation of the Wegner-Wilson loop in the stochastic vacuum model

B. The scattering of gluons

C. The scattering of baryons 


\section{Introduction}

In these lectures I would like to review some ideas on the way nonperturbative QCD may manifest itself in high energy collisions. Thus we will be concerned with strong interactions where we claim to know the fundamental Lagrangian for a long time now [1]:

$$
\mathcal{L}_{\mathrm{QCD}}(x)=-\frac{1}{4} G_{\lambda \rho}^{a}(x) G^{\lambda \rho a}(x)+\sum_{q} \bar{q}(x)\left(i \gamma^{\lambda} D_{\lambda}-m_{q}\right) q(x) .
$$

Here $q(x)$ are the quark fields for the various quark flavours $(q=u, d, s, c, b, t)$ with masses $m_{q}$. We denote the gluon potentials by $G_{\lambda}^{a}(x)(a=1, \ldots, 8)$ and the gluon field strengths by

$$
G_{\lambda \rho}^{a}(x)=\partial_{\lambda} G_{\rho}^{a}(x)-\partial_{\rho} G_{\lambda}^{a}(x)-g f_{a b c} G_{\lambda}^{b}(x) G_{\rho}^{c}(x),
$$

where $g$ is the strong coupling constant and $f_{a b c}$ are the structure constants of $\mathrm{SU}(3)$. The covariant derivative of the quark fields is

$$
D_{\lambda} q(x)=\left(\partial_{\lambda}+i g G_{\lambda}^{a} \frac{\lambda_{a}}{2}\right) q(x)
$$

with $\lambda_{a}$ the Gell-Mann matrizes of the $\mathrm{SU}(3)$ group. The gluon potential and field strength matrizes are defined as

$$
\begin{aligned}
G_{\lambda}(x): & =G_{\lambda}^{a}(x) \frac{\lambda_{a}}{2} \\
G_{\lambda \rho}(x): & =G_{\lambda \rho}^{a}(x) \frac{\lambda_{a}}{2} .
\end{aligned}
$$

The Lagrangian (1.1) is invariant under $\mathrm{SU}(3)$ gauge transformations. Let $x \rightarrow U(x)$ be an arbitrary matrix function, where for fixed $x$ the $U(x)$ are $\mathrm{SU}(3)$ matrices:

$$
\begin{aligned}
U(x) U^{\dagger}(x) & =1 \\
\operatorname{det} U(x) & =1
\end{aligned}
$$

With the transformation laws:

$$
q(x) \rightarrow U(x) q(x)
$$




$$
G_{\lambda}(x) \rightarrow U(x) G_{\lambda}(x) U^{\dagger}(x)-\frac{i}{g} U(x) \partial_{\lambda} U^{\dagger}(x)
$$

we find

$$
G_{\lambda \rho}(x) \rightarrow U(x) G_{\lambda \rho}(x) U^{\dagger}(x)
$$

and invariance of $\mathcal{L}_{\mathrm{QCD}}$ :

$$
\mathcal{L}_{\mathrm{QCD}}(x) \rightarrow \mathcal{L}_{\mathrm{QCD}}(x)
$$

If we want to derive results from the Lagrangian (1.1), we face problems, the most notable being that $\mathcal{L}_{\mathrm{QCD}}$ is expressed in terms of quark and gluon fields whose quanta have not been observed as free particles. In the real world we observe only hadrons, colourless objects, quark and gluons are permanently confined. Nevertheless it has been possible in some cases to derive first principle results which can be compared with experiment, starting from $\mathcal{L}_{\mathrm{QCD}}$ (1.1). These are in essence the following:

(1) Pure short-distance phenomena: Due to asymptotic freedom [2] the QCD coupling constant becomes small in this regime and one can make reliable perturbative calculations. Examples of pure short distance processes are for instance the total cross section for electron-positron annihilation into hadrons and the total hadronic decay rate of the $Z$-boson.

(2) Pure long-distance phenomena: Here one is in the nonperturbative regime of QCD and one has to use numerical methods to obtain first principle results from $\mathcal{L}_{\mathrm{QCD}}$, or rather from the lattice version of $\mathcal{L}_{\mathrm{QCD}}$ introduced by Wilson [3]. Today, Monte Carlo simulations of lattice QCD are a big industry among theorists. Typical quantities one can calculate in this way are hadron masses and other low energy hadron properties. (For an up-to-date account of these methods cf. [4]).

There is a third regime of hadronic phenomena, hadron-hadron collisions, which are - apart from very low energy collisions - neither pure long-distance nor pure short-distance phenomena. Thus, none of the above-mentioned theoretical methods apply directly. Traditionally one classifies high-energy hadron-hadron collisions as "hard" and "soft" ones:

(3) High energy hadron-hadron collisions:

(a) hard collisions,

(b) soft collisions.

A typical hard reaction is the Drell-Yan process, e.g. 


$$
\begin{aligned}
\pi^{-}+N \rightarrow & \gamma^{*}+X \\
& \hookrightarrow \ell^{+} \ell^{-}
\end{aligned}
$$

where $\ell=e, \mu$. All energies and momentum transfers are assumed to be large. However, the masses of the $\pi^{-}$and $N$ in the initial state stay fixed and thus we are not dealing with a pure short distance phenomenon.

In the reaction (1.9) we claim to see directly the fundamental quanta of the theory, the partons, i.e. the quarks and gluons, in action (cf. Fig. 1). In the usual theoretical framework for hard reactions, the QCD improved parton model (cf. e.g. [5]), one describes the reaction of the partons, in the Drell-Yan case the $q \bar{q}$ annihilation into a virtual photon, by perturbation theory. This should be reliable, since the parton process involves only high energies and high momentum transfers. All the long distance physics due to the bound state nature of the hadrons is then lumped into parton distribution functions of the participating hadrons. This is called the factorization hypothesis, which after early investigations of soft initial and final state interactions [6] was formulated and studied in low orders of QCD perturbation theory in [7]. Subsequently, great theoretical effort has gone into proving factorization in the framework of QCD perturbation theory [8]- [10]. The result seems to be that factorization is most probably correct there (cf. the discussion in [11]). However, it is legitimate to ask if factorization is respected also by nonperturbative effects. To my knowledge this question was first asked in [12] - 14]. In Sect. 4 of these lectures I will come back to this question and will argue that there may be evidence for a breakdown of factorization in the Drell-Yan reaction due to $\mathrm{QCD}$ vacuum effects.

Let us consider now soft high energy collisions. A typical reaction in this class is proton-proton elastic scattering:

$$
p+p \rightarrow p+p
$$

at c.m. energies $E_{\mathrm{cm}}=\sqrt{s} \gtrsim 5 \mathrm{GeV}$ say and small momentum transfers

$\sqrt{|t|}=|q| \lesssim 1 \mathrm{GeV}$. Here we have two scales, one staying finite, one going to infinity:

$$
\begin{aligned}
E_{\mathrm{cm}} & \rightarrow \infty, \\
|q| & \lesssim 1 \mathrm{GeV} .
\end{aligned}
$$


Thus, none of the above mentioned calculational methods is directly applicable. Indeed, most theoretical papers dealing with reactions in this class develop and apply models which are partly older than QCD, partly QCD "motivated". Let me list some models for hadron-hadron elastic scattering at high energies:

geometric [15],

eikonal [16],

additive quark model [17],

Regge poles [18],

topological expansions and strings [19],

valons [20],

leading $\log$ summations [21],

two-gluon exchange [22],

the Donnachie-Landshoff model for the "soft Pomeron" [23.

It would be a forbidding task to collect all references in this field. The references given above should thus only be considered as representative ones. In addition I would like to mention the inspiring general field theoretic considerations for high energy scattering and particle production by Heisenberg [24] and the impressive work by Cheng and Wu on high energy behaviour in field theories in the framework of perturbative calculations [25].

I will now argue that the theoretical description of measurable quantities of soft high energy reactions like the total cross sections should involve in an essential way nonperturbative QCD. To see this, consider massless pure gluon theory where all "hadrons" are massive glueballs. Then we know from the renormalization group analysis that the glueball masses must behave as

$$
m_{\text {glueball }} \propto M e^{-c / g^{2}(M)}
$$

for $M \rightarrow \infty$, i.e. for $g(M) \rightarrow 0$, due to asymptotic freedom. Here $M$ is the renormalization scale, $g(M)$ is the QCD coupling strength at this scale and $c$ is a constant:

$$
\begin{aligned}
& \frac{g^{2}(M)}{4 \pi^{2}} \longrightarrow \frac{12}{33 \ln \left(M^{2} / \Lambda^{2}\right)} \text { for } \quad M \rightarrow \infty, \\
& c=\frac{8 \pi^{2}}{11}, \\
& \Lambda: \quad \text { QCD scale parameter. }
\end{aligned}
$$


Masses in massless Yang-Mills theory are a purely nonperturbative phenomenon, due to "dimensional transmutation". Scattering of glueball-hadrons in massless pure gluon theory should look very similar to scattering of hadrons in the real world, with finite total cross sections, amplitudes with analytic $t$ dependence etc. At least, this would be my expectation. If the total cross section $\sigma_{\text {tot }}$ has a finite limit as $s \rightarrow \infty$ we must have from the same renormalization group arguments:

$$
\lim _{s \rightarrow \infty} \sigma_{\text {tot }}(s) \propto M^{-2} e^{2 c / g^{2}(M)}
$$

for $g(M) \rightarrow 0$. In this case, the total cross sections in pure gluon theory are also nonperturbative objects! It is easy to see that this conclusion is not changed if $\sigma_{\text {tot }}(s)$ has a logarithmic behaviour with $s$ for $s \rightarrow \infty$, e.g.

$$
\sigma_{\text {tot }}(s) \rightarrow \text { const } \times(\log s)^{2} .
$$

I would then expect that also in full QCD total cross sections are nonperturbative objects, at least as far as hadrons made out of light quarks are concerned.

Some time ago P.V. Landshoff and myself started to think about a possible connection between the nontrivial vacuum structure of QCD - a typical nonperturbative phenomenon - and soft high energy reactions [26]. In the following I will first review some common folklore on the QCD vacuum and discuss in more detail the so-called "stochastic vacuum model". I will then sketch possible consequences of these ideas for high energy collisions.

\section{The QCD Vacuum}

According to current theoretical prejudice the vacuum state in QCD has a very complicated structure [27]-37]. It was first noted by Savvidy [27] that by introducing a constant chromomagnetic field

$$
\mathbf{B}^{a}=\mathbf{n} \eta^{a} B,(a=1, \ldots, 8),
$$

into the perturbative vacuum one can lower the vacuum-energy density $\varepsilon(B)$. Here $\mathbf{n}$ and $\eta^{a}$ are constant unit vectors in ordinary and colour space. The result of his one-loop calculation was

$$
\varepsilon(B)=\frac{1}{2} B^{2}+\frac{\beta_{0} g^{2}}{32 \pi^{2}} B^{2}\left[\ln \frac{B}{M^{2}}-\frac{1}{2}\right]
$$


where $g$ is the strong coupling constant, $M$ is again the renormalization scale, and $\beta_{0}$ is given by the lowest order term in the Callan-Symanzik $\beta$-function:

$$
M \frac{\mathrm{d} g(M)}{\mathrm{d} M}=: \beta(g)=-\frac{\beta_{0}}{16 \pi^{2}} g^{3}+\ldots
$$

For 3 colours and $f$ flavours:

$$
\beta_{0}=11-\frac{2}{3} f .
$$

Thus, as long as we have asymptotic freedom, i.e. for $f \leq 16$, the energy density $\varepsilon(B)$ looks as indicated schematically in Fig. 2 and has its minimum for $B=B_{\text {vac }} \neq 0$. Therefore, we should expect the QCD-vacuum to develop spontaneously a chromomagnetic field, the situation being similar to that in a ferromagnet below the Curie temperature where we have spontaneous magnetization.

Of course, the vacuum state in QCD has to be relativistically invariant and cannot have a preferred direction in ordinary space and colour space. What has been considered [33] are states composed of domains with random orientation of the gluon-field strength (Fig. 3). This is analogous to Weiss domains in a ferromagnet. The vacuum state should then be a suitable linear superposition of states with various domains and orientation of the fields inside the domains. This implies that the orientation of the fields in the domains as well as the boundaries of the domains will fluctuate.

A very detailed picture for the QCD vacuum along these lines has been developed in ref. [33]. I cannot refrain from comparing this modern picture of the QCD vacuum (Fig. 4a) with the "modern picture" of the ether developed by Maxwell more than 100 years ago [38] (Fig. 4b). The analogy is quite striking and suggests to me that with time passing on we may also be able to find simpler views on the QCD vacuum. Remember that Einstein made great progress by eliminating the ether from electrodynamics. In the following we will adopt the picture of the QCD vacuum as developed in refs. [27]-[34], [36] and outlined above as a working hypothesis.

Let me now come to the values for the field strengths $\mathbf{E}^{a}$ and $\mathbf{B}^{a}$ in the vacuum. These must also be determined by $\Lambda$, the QCD scale parameter, the only dimensional parameter in QCD if we disregard the quark masses. Therefore, we must have on dimensional grounds for the renormalization 
group invariant quantity $(g B)^{2}$

$$
(g B)^{2} \propto \Lambda^{4}
$$

But we have much more detailed information on the values of these field strengths due to the work of Shifman, Vainshtein, and Zakharov (SVZ), who introduced the gluon condensate and first estimated its value using sum rules for charmonium states [30]:

$$
\begin{aligned}
<0\left|\frac{g^{2}}{4 \pi^{2}} G_{\mu \nu}^{a}(x) G^{\mu \nu a}(x)\right| 0> & \equiv<0\left|\frac{g^{2}}{2 \pi^{2}}\left(\mathbf{B}^{a}(x) \mathbf{B}^{a}(x)-\mathbf{E}^{a}(x) \mathbf{E}^{a}(x)\right)\right| 0> \\
& \equiv G_{2}=(2.4 \pm 1.1) \cdot 10^{-2} \mathrm{GeV}^{4} \\
& =(335-430 \mathrm{MeV})^{4}
\end{aligned}
$$

Here we quote numerical values as given in the review [39]. A simple analysis shows that this implies

$$
<0\left|g^{2} \mathbf{B}^{a}(x) \mathbf{B}^{a}(x)\right| 0>=-<0\left|g^{2} \mathbf{E}^{a}(x) \mathbf{E}^{a}(x)\right| 0>=\pi^{2} G_{2} \simeq(700 \mathrm{MeV})^{4} .
$$

To prove eq. (2.7) we note that Lorentz- and parity-invariance require the vacuum expectation value of the uncontracted product of two gluon field strengths to be of the form

$$
<0\left|\frac{g^{2}}{4 \pi^{2}} G_{\mu \nu}^{a}(x) G_{\rho \sigma}^{b}(x)\right| 0>=\left(g_{\mu \rho} g_{\nu \sigma}-g_{\mu \sigma} g_{\nu \rho}\right) \delta^{a b} \frac{G_{2}}{96}
$$

where $G_{2}$ is the same constant as in (2.6). Taking appropriate contractions leads to (2.6) and (2.7).

We find that $<0\left|\mathbf{B}^{a}(x) \mathbf{B}^{a}(x)\right| 0>$ is positive, $<0\left|\mathbf{E}^{a}(x) \mathbf{E}^{a}(x)\right| 0>$ negative! This can happen because we are really considering products of field operators, normal-ordered with respect to the perturbative vacuum. The interpretation of eq. (2.7) is, therefore, that the B-field fluctuates with bigger amplitude, the E-field with smaller amplitude than in the perturbative vacuum state.

What about the size $a$ of the colour domains and the fluctuation times $\tau$ of the colour fields? On dimensional grounds we must have

$$
a \sim \tau \sim \Lambda^{-1}
$$


A detailed model for the QCD vacuum incorporating the gluon condensate idea and a fall-off of the correlation of two field strengths with distance was proposed in [40]: the "stochastic vacuum model" (SVM). In the following we will discuss the basic assumptions of the model and then apply it to derive the area law for the Wegner-Wilson loop, i.e. confinement of static quarks. For the rest of this section we will work in Euclidean space-time. To accomplish the analytic continuation from Minkowski to Euclidean space-time we have to make the following replacements for $x^{\lambda}$ and $G^{\lambda}$ (cf. (1.4)):

$$
\begin{aligned}
& x^{0} \rightarrow-i X_{4}, \\
& \mathbf{x} \rightarrow \mathbf{X} \\
& G^{0} \rightarrow i \mathcal{G}_{4}, \\
& \mathbf{G} \rightarrow-\mathcal{G} .
\end{aligned}
$$

Here $X=\left(\mathbf{X}, X_{4}\right)$ denotes an Euclidean space-time point and $\mathcal{G}_{\alpha}(\alpha=$ $1, \ldots, 4)$ the Euclidean gluon potential. With (2.10) we get

$$
\begin{aligned}
& (x \cdot y) \rightarrow-(X \cdot Y)=-X_{\alpha} Y_{\alpha}, \\
& -i g \int \mathrm{d} x^{\mu} G_{\mu}(x) \rightarrow-i g \int \mathrm{d} X_{\alpha} \mathcal{G}_{\alpha}(X), \\
& G^{0 j} \rightarrow-i \mathcal{G}_{4 j}, \\
& G^{j k} \rightarrow \mathcal{G}_{j k},
\end{aligned}
$$

where $1 \leq j, k \leq 3, \quad 1 \leq \alpha, \beta \leq 4$ and

$$
\mathcal{G}_{\alpha \beta}=\partial_{\alpha} \mathcal{G}_{\beta}-\partial_{\beta} \mathcal{G}_{\alpha}+i g\left[\mathcal{G}_{\alpha}, \mathcal{G}_{\beta}\right]
$$

is the Euclidean gluon field strength tensor.

\subsection{Connectors}

Consider classical gluon fields in Euclidean space-time. Let $X, Y$ be two points there and $C_{X}$ a curve from $X$ to $Y$ (Fig. 5). We define the connector, the non-abelian generalization of the "Schwinger string" 41] of QED as

$$
V\left(Y, X ; C_{X}\right)=\mathrm{P}\left\{\exp \left[-i g \int_{C_{X}} \mathrm{~d} Z_{\alpha} \mathcal{G}_{\alpha}(Z)\right]\right\}
$$


where $\mathrm{P}$ means path ordering. The connector can be obtained as solution of a differential equation. Let

$$
\begin{aligned}
& \tau \rightarrow Z(\tau) \\
& \tau_{1} \leq \tau \leq \tau_{2}, \\
& Z\left(\tau_{1}\right)=X, \quad Z\left(\tau_{2}\right)=Y
\end{aligned}
$$

be a parametrization of $C_{X}$. Consider the differential equation for a $3 \times 3$ matrix function $V(\tau)$ :

$$
\frac{\mathrm{d}}{\mathrm{d} \tau} V(\tau)=-i g \frac{\mathrm{d} Z_{\alpha}(\tau)}{\mathrm{d} \tau} \mathcal{G}_{\alpha}(Z(\tau)) V(\tau),
$$

with the boundary condition

$$
V\left(\tau_{1}\right)=11
$$

The solution of (2.15), (2.16) gives for $\tau=\tau_{2}$ just the connector (2.13).

Under a gauge transformation

$$
\mathcal{G}_{\alpha}(X) \rightarrow U(X) \mathcal{G}_{\alpha}(X) U^{\dagger}(X)-\frac{i}{g} U(X) \partial_{\alpha} U^{\dagger}(X)
$$

where $U(X) \in S U(3)$, we have

$$
V\left(Y, X ; C_{X}\right) \rightarrow U(Y) V\left(Y, X ; C_{X}\right) U^{-1}(X) .
$$

The connector can be used to "shift" various objects from one space-time point to another in a gauge-covariant way. We define for instance the field strength tensor shifted from $X$ to $Y$ along $C_{X}$ as

$$
\hat{\mathcal{G}}_{\alpha \beta}\left(Y, X ; C_{X}\right):=V\left(Y, X ; C_{X}\right) \mathcal{G}_{\alpha \beta}(X) V^{-1}\left(Y, X ; C_{X}\right) .
$$

Under a gauge transformation $\hat{\mathcal{G}}_{\alpha \beta}\left(Y, X ; C_{X}\right)$ transforms like a field strength tensor at $Y$ :

$$
\hat{\mathcal{G}}_{\alpha \beta}\left(Y, X ; C_{X}\right) \rightarrow U(Y) \hat{\mathcal{G}}_{\alpha \beta}\left(Y, X ; C_{X}\right) U^{-1}(Y) .
$$

Connectors can, of course, be defined for arbitrary SU(3) representations, not only for the fundamental one used in (2.13). Let $T_{a}(a=1, \ldots 8)$ be the generators of $\mathrm{SU}(3)$ in some arbitrary unitary representation $R$ where

$$
\left[T_{a}, T_{b}\right]=i f_{a b c} T_{c}
$$


We define the connector for this representation by:

$$
V_{R}\left(Y, X ; C_{X}\right):=\mathrm{P} \exp \left[-i g \int_{C_{X}} \mathrm{~d} Z_{\alpha} \mathcal{G}_{\alpha}^{a}(Z) T_{a}\right]
$$

We list some basic properties of connectors:

(i) For 2 adjoining curves $C_{1}, C_{2}$ (Fig. 6), the connectors are multiplied:

$$
V_{R}\left(X_{3}, X_{1} ; C_{2}+C_{1}\right)=V_{R}\left(X_{3}, X_{2} ; C_{2}\right) \cdot V_{R}\left(X_{2}, X_{1} ; C_{1}\right)
$$

(ii) Let $C$ be a curve from $X$ to $Y$ and $\bar{C}$ the same curve but oriented in inverse direction, running from $Y$ to $X$ (Fig. 6). Then

$$
\begin{gathered}
V_{R}(X, Y ; \bar{C}) V_{R}(Y, X ; C)=11, \\
V_{R}^{\dagger}(Y, X ; C)=V_{R}(X, Y ; \bar{C}) .
\end{gathered}
$$

The product of the connectors for the path and the reverse path is equal to the unit matrix. The reversal of the path is equivalent to hermitian conjugation.

We leave the proof of (2.23)-(2.25) as an exercise for the reader.

\subsection{The non-abelian Stokes theorem}

In this subsection we will derive the non-abelian generalization of the Stokes theorem [42]. Consider a surface $S$ in Euclidean space time with boundary $C=\partial S$. Let $X$ be some point on $C$ as indicated in Fig. 7 and consider the connector (2.22) from $X$ back to $X$ along $C$ :

$$
V_{R}(X, X ; C)=\mathrm{P} \exp \left[-i g \int_{C} \mathrm{~d} Z_{\alpha} \mathcal{G}_{\alpha}^{a}(Z) T_{a}\right]
$$

The problem is to transform this line integral into a surface integral.

We start by considering a point $Z_{1}$ in $S$ and a small plaquette formed by curves $C_{1}, . ., C_{4}$ where one corner point is $Z_{1}$ (Fig. 7). We choose a coordinate system on $S$ in the neighbourhood of $Z_{1}$ :

$$
(u, v) \rightarrow Z(u, v)
$$

such that

$$
\begin{aligned}
& Z_{1}=Z(0,0) \\
& Z_{2}=Z(\Delta u, 0) \\
& Z_{3}=Z(\Delta u, \Delta v) \\
& Z_{4}=Z(0, \Delta v)
\end{aligned}
$$


and the curves $C_{1}, C_{3}\left(C_{2}, C_{4}\right)$ correspond to lines $v=$ const. ( $u=$ const.). The matrix representing the line integral around the small plaquette is

$$
U_{R}(\Delta u, \Delta v):=V_{R}\left(C_{4}\right) V_{R}\left(C_{3}\right) V_{R}\left(C_{2}\right) V_{R}\left(C_{1}\right) .
$$

We want to make a Taylor expansion of $U_{R}(\Delta u, \Delta v)$ in $\Delta u$ and $\Delta v$. From (2.24) we find immediately that

$$
U_{R}(0, \Delta v)=U_{R}(\Delta u, 0)=11 .
$$

Thus the lowest order term in the expansion after the zeroth order is proportional to $\Delta u \cdot \Delta v$ and we get easily:

$$
\begin{aligned}
& U_{R}(\Delta u, \Delta v)=11-i g \frac{1}{2} \Delta \sigma_{\alpha \beta} G_{\alpha \beta}^{a}\left(Z_{1}\right) T_{a} \\
& +O\left(\Delta u^{2} \Delta v, \Delta u \Delta v^{2}\right)
\end{aligned}
$$

where

$$
\Delta \sigma_{\alpha \beta}=\Delta u \Delta v \frac{\partial\left(Z_{\alpha}, Z_{\beta}\right)}{\partial(u, v)} .
$$

In the limit $\Delta u, \Delta v \rightarrow 0 \Delta \sigma_{\alpha \beta}$ becomes the surface element $d \sigma_{\alpha \beta}$ of the plaquette.

The next step is to choose an arbitrary point $Y$, the reference point, on the surface $S$ and to draw a fan-type net on $S$ as a spider would do (Fig. 8). The system of curves of the net consists of the curve $C_{X}$ running from $X$ to $Y$, then $\bar{C}_{Z_{1}}$ from $Y$ to $Z_{1}$, then around a small plaquette at $Z_{1}$, back to $Y$ along $C_{Z_{1}}$ and so on. The final curve is $\bar{C}_{X}$ from $Y$ to $X$. Apart from the initial and final curves $C_{X}$ and $\bar{C}_{X}$ we have a system of plaquettes with "handles" connecting them to $Y$. With the help of (2.23)-(2.25) we see that the connector along the whole net is equivalent to the original connector (2.26).

$$
\begin{aligned}
& V_{R}(X, X ; C)=V_{R}\left(X, Y ; \bar{C}_{X}\right) \cdot \text { product of connectors } \\
& \text { for the plaquettes with handles } \cdot V_{R}\left(Y, X ; C_{X}\right) .
\end{aligned}
$$

Let us consider one plaquette with handle, for instance the one at $Z_{n}$ in Fig. 8. For this contribution to (2.33) we get

$$
\begin{aligned}
& V_{R}\left(Y, Z_{n} ; C_{Z_{n}}\right) V_{R}\left(\text { plaquette at } Z_{n}\right) V_{R}\left(Z_{n}, Y ; \bar{C}_{Z_{n}}\right) \\
& =V_{R}\left(Y, Z_{n} ; C_{Z_{n}}\right)\left[1-i g \frac{1}{2} \Delta \sigma_{\alpha \beta} \mathcal{G}_{\alpha \beta}^{a}\left(Z_{n}\right) T_{a}+\cdots\right] V_{R}\left(Z_{n}, Y ; \bar{C}_{Z_{n}}\right) \\
& =11-i g \frac{1}{2} \Delta \sigma_{\alpha \beta} \hat{\mathcal{G}}_{\alpha \beta}^{a}\left(Y, Z_{n} ; C_{Z_{n}}\right) T_{a}+\cdots
\end{aligned}
$$


Here we use (2.31) and the shifted field strengths as defined in (2.19). We leave it as an excercise to the reader to show that (2.19) implies

$$
\begin{aligned}
& V_{R}\left(Y, Z_{n} ; C_{Z_{n}}\right) \mathcal{G}_{\alpha \beta}^{a}\left(Z_{n}\right) T_{a} V_{R}\left(Z_{n}, Y ; \bar{C}_{Z_{n}}\right) \\
& =\hat{\mathcal{G}}^{a}\left(Y, Z_{n} ; C_{Z_{n}}\right) T_{a}
\end{aligned}
$$

for arbitrary representation $R$ of $S U(3)$.

Inserting (2.34) in (2.33) and summing up the contribution of all plaquettes with handles, where we have of course to respect the ordering, we get in the limit that the net is infinitesimally fine:

$$
\begin{aligned}
& V_{R}(X, X ; C)=V_{R}\left(X, Y ; \bar{C}_{X}\right) . \\
& \mathrm{P} \exp \left[-i \frac{g}{2} \int_{S} \mathrm{~d} \sigma_{\alpha \beta} \hat{\mathcal{G}}_{\alpha \beta}^{a}\left(Y, Z ; C_{Z}\right) T_{a}\right] V_{R}\left(Y, X ; C_{X}\right) .
\end{aligned}
$$

Here $P$ denotes the ordering on the whole surface as implied by the net. Usually one takes the trace in (2.36) which leads with (2.24) to

$$
\operatorname{Tr} V_{R}(X, X ; C)=\operatorname{Tr} \mathrm{P} \exp \left[-i \frac{g}{2} \int_{S} \mathrm{~d} \sigma_{\alpha \beta} \hat{\mathcal{G}}_{\alpha \beta}^{a}\left(Y, Z ; C_{Z}\right) T_{a}\right] .
$$

This is the desired non-abelian version of Stokes' theorem. We leave it to the reader as an exercise to show that for the abelian case (2.37) reduces to the conventional Stokes theorem.

\subsection{The cumulant expansion}

As a last mathematical tool for making calculations with the SVM we discuss the cumulant expansion 43]. Consider functions

$$
\tau \rightarrow B(\tau)
$$

on the interval $O \leq \tau \leq 1$ where $B(\tau)$ are quadratic matrices. We assume that an averaging procedure over products of the functions $B(\cdot)$ is defined:

$$
E\left(B\left(\tau_{1}\right)\right), E\left(B\left(\tau_{1}\right) B\left(\tau_{2}\right)\right), \ldots
$$

We consider first the case that all averages $E(\cdot)$ are $c$ numbers and that

$$
E(1)=1
$$


Let us consider the expectation value of the $\tau$-ordered exponential:

$$
f(t):=E\left(\mathrm{P} \exp \left[t \int_{0}^{1} \mathrm{~d} \tau B(\tau)\right]\right),
$$

where $t \in \mathcal{C}$. The cumulant expansion asserts that $\ln f(t)$ can be expanded as

$$
\ln f(t)=\sum_{n=1}^{\infty} \frac{t^{n}}{n !} \int_{0}^{1} \mathrm{~d} \tau_{1} \ldots \int_{0}^{1} \mathrm{~d} \tau_{n} K_{n}\left(\tau_{1}, \ldots, \tau_{n}\right),
$$

where the $n$-th cumulant $K_{n}\left(\tau_{1}, \ldots, \tau_{n}\right)$ is a symmetric function of its arguments. A frequently used notation for the $K_{n}$ is:

$$
K_{n}\left(\tau_{1}, \ldots, \tau_{n}\right) \equiv\left\langle\left\langle B\left(\tau_{1}\right) \ldots B\left(\tau_{n}\right)\right\rangle\right\rangle .
$$

To prove (2.41) we proceed as follows. Expanding in powers of $t$ on the r.h.s. of $(2.40)$ we get

$$
f(t)=1+\sum_{n=1}^{\infty} \frac{t^{n}}{n !} \mathcal{B}_{n}
$$

where

$$
\mathcal{B}_{n}=\int_{0}^{1} \mathrm{~d} \tau_{1} \ldots \int_{0}^{1} \mathrm{~d} \tau_{n} E\left(\mathrm{P}\left(B\left(\tau_{1}\right) \ldots B\left(\tau_{n}\right)\right)\right) .
$$

Now we expand $\ln f(t)$ :

$$
\ln f(t)=\sum_{n=1}^{\infty} \frac{t^{n}}{n !} \mathcal{K}_{n},
$$

where the expansion coefficients $\mathcal{K}_{n}$ are obtained from:

$$
\begin{aligned}
f(t) & =\exp (\ln f(t)), \\
1+\sum_{n=1}^{\infty} \frac{t^{n}}{n !} \mathcal{B}_{n} & =\exp \left[\sum_{n=1}^{\infty} \frac{t^{n}}{n !} \mathcal{K}_{n}\right] .
\end{aligned}
$$

From this we obtain the $\mathcal{K}_{n}$ as solution of the following system of equations:

$$
\begin{aligned}
\mathcal{B}_{1}= & \mathcal{K}_{1}, \\
\mathcal{B}_{2}= & \mathcal{K}_{2}+\mathcal{K}_{1}^{2}, \\
\mathcal{B}_{3}= & \mathcal{K}_{3}+\frac{3}{2}\left(\mathcal{K}_{2} \mathcal{K}_{1}+\mathcal{K}_{1} \mathcal{K}_{2}\right)+\mathcal{K}_{1}^{3} \\
\mathcal{B}_{4}= & \mathcal{K}_{4}+2\left(\mathcal{K}_{3} \mathcal{K}_{1}+\mathcal{K}_{1} \mathcal{K}_{3}\right)+3 \mathcal{K}_{2}^{2}+2\left(\mathcal{K}_{2} \mathcal{K}_{1}^{2}+\mathcal{K}_{1} \mathcal{K}_{2} \mathcal{K}_{1}+\mathcal{K}_{1}^{2} \mathcal{K}_{2}\right)+\mathcal{K}_{1}^{4}, \\
& \cdots
\end{aligned}
$$


Clearly, this system can be inverted and we get $\mathcal{K}_{n}$ as sum of monomials of the form

$$
\mathcal{B}_{i_{1}} \cdot \mathcal{B}_{i_{2}} \ldots \mathcal{B}_{i_{k}}
$$

where

$$
\sum_{j=1}^{k} i_{j}=n .
$$

Using (2.44), every monomial 2.48) can be written as $n$-fold integral over $\tau_{1}, \ldots, \tau_{n}$ with $0 \leq \tau_{j} \leq 1(j=1, \ldots, n)$. Since the integration domain is symmetric under arbitrary permutations of $\tau_{1}, \ldots, \tau_{n}$ we can symmetrize the integrand completely. In this way we get

$$
\mathcal{K}_{n}=\int_{0}^{1} \mathrm{~d} \tau_{1} \ldots \int_{0}^{1} \mathrm{~d} \tau_{n} K_{n}\left(\tau_{1}, \ldots, \tau_{n}\right)
$$

where $K_{n}$ is a totally symmetric function of its arguments. Inserting (2.50) in (2.45) we get the cumulant expansion (2.41), q.e.d. Explicitly we find for the first few cumulants:

$$
\begin{aligned}
K_{1}(1)= & E(B(1)) \\
K_{2}(1,2)= & E(\mathrm{P}(B(1) B(2))) \\
& -\frac{1}{2} E(B(1)) E(B(2))-\frac{1}{2} E(B(2)) E(B(1)) \\
K_{3}(1,2,3)= & E(\mathrm{P}(B(1) B(2) B(3))) \\
& -\frac{1}{2}[E(\mathrm{P}(B(1) B(2))) E(B(3)) \\
& +E(B(1)) E(\mathrm{P}(B(2) B(3))) \\
& +\operatorname{cycl} . \text { perm. }] \\
& +\frac{1}{3}[E(B(1)) E(B(2)) E(B(3))+\text { perm. }] \\
& \ldots
\end{aligned}
$$

Here we write as a shorthand notation $K_{1}(1) \equiv K_{1}\left(\tau_{1}\right), B(1) \equiv B\left(\tau_{1}\right)$ etc.

The cumulant expansion (2.41) has the so-called "cluster" property: Let us assume that the expectation values of the P-ordered products factorize

$$
E(\mathrm{P}(B(1) \ldots B(n)))=\frac{1}{n !}\{E(B(1)) \ldots E(B(n))+\text { perm. }\}
$$


for all $n \geq 2$ and all

$$
\left|\tau_{i}-\tau_{j}\right| \geq \tau_{\min } \quad(i \neq j) .
$$

We can then show that the cumulants for $n \geq 2$ vanish:

$$
K_{n}(1, \ldots, n)=0, \quad(n \geq 2)
$$

if the $\tau_{i}$ satisfy (2.53).

To prove (2.54) we show first that it is true for $n=2$. Indeed, from (2.51) and (2.52) we get for $\left|\tau_{1}-\tau_{2}\right| \geq \tau_{\min }$

$$
\begin{aligned}
K_{2}(1,2)= & \frac{1}{2 !}\{E(B(1)) E(B(2))+\text { perm. }\} \\
& -\frac{1}{2} E(B(1)) E(B(2))-\frac{1}{2} E(B(2)) E(B(1)) \\
= & 0 .
\end{aligned}
$$

Now we proceed by mathematical induction. Assume that (2.54) has been shown for all $k$ with $2 \leq k \leq n-1$. We have from (2.47):

$$
\begin{aligned}
K_{n}(1, \ldots, n)= & E(\mathrm{P}(B(1) \ldots B(n))) \\
- & \frac{1}{n !}\left[K_{1}(1) \ldots K_{1}(n)+\text { perm. }\right] \\
+ & {\left[\text { symmetrized products of cumulants } K_{k}(1, \ldots, k)\right.} \\
& \quad \text { with } 1 \leq k \leq n-1]
\end{aligned}
$$

but at least one factor with $k \geq 2$. With (2.52) we get now immediately $K_{n}(1, \ldots, n)=0$ in the region defined by (2.53), q.e.d.

Up to now we have assumed the expectation values $E(\cdot)$ to be $c$-numbers. In this case many of the formulae (2.47) - (2.56) can be simplified by using

$$
\begin{aligned}
& E(B(1)) E(B(2))=E(B(2)) E(B(1)), \\
& \text { etc. }
\end{aligned}
$$

We have, on purpose, not used such commutativity relations, since we are now going to generalize the cumulant expansion to the case where

$$
E(B(1)), E(B(1) B(2)), \ldots
$$


are themselves quadratic matrix valued expectation values with

$$
E(11)=11 .
$$

Then we have, of course, in general, no more the commutativity relations (2.57). But all formulae (2.40) - (2.56) are written in such a way that they remain true also for the case of matrix valued expectation values $E(\cdot)$.

\subsection{The basic assumptions of the stochastic vacuum model}

The basic object of the SVM is the correlator of two field strengths shifted to a common reference point. Let $X, X^{\prime}$ be two points in Euclidean spacetime, $Y$ a reference point and $C_{X}, C_{X^{\prime}}$ curves from $X$ to $Y$ and $X^{\prime}$ to $Y$, respectively (Fig. 5). We consider the shifted field strengths as defined in (2.19) and the vacuum expectation value of their product in the sense of Euclidean QFT:

$$
\left\langle\frac{g^{2}}{4 \pi^{2}}\left[\hat{\mathcal{G}}_{\mu \nu}^{a}\left(Y, X ; C_{X}\right) \hat{\mathcal{G}}_{\rho \sigma}^{b}\left(Y, X^{\prime} ; C_{X^{\prime}}\right)\right]\right\rangle=: \frac{1}{4} \delta^{a b} F_{\mu \nu \rho \sigma}\left(X, X^{\prime}, Y ; C_{X}, C_{X^{\prime}}\right) .
$$

Here (2.20) and colour conservation allow us to write the r.h.s. of (2.60) proportional to $\delta^{a b}$. It is easy to see that $F_{\mu \nu \rho \sigma}$ depends only on $X, X^{\prime}$ and the curve $C_{X}+\bar{C}_{X^{\prime}}$ connecting them; i.e. the reference point $Y$ can be freely shifted on the connecting curve. In the SVM one makes now the

strong assumption that the correlator (2.60) even does not depend on the connecting curve at all:

- Ass. 1: $F_{\mu \nu \rho \sigma}$ is independent of the reference point $Y$ and of the curves $C_{X}$ and $C_{X^{\prime}}$.

Translational, $O(4)$ - and parity invariance require then the correlator (2.60) to be of the following form:

$$
\begin{aligned}
& F_{\mu \nu \rho \sigma}=F_{\mu \nu \rho \sigma}(Z)=\frac{1}{24} G_{2}\left\{\left(\delta_{\mu \rho} \delta_{\nu \sigma}-\delta_{\mu \sigma} \delta_{\nu \rho}\right) \kappa D\left(-Z^{2}\right)\right. \\
& \left.+\frac{1}{2}\left[\frac{\partial}{\partial Z_{\nu}}\left(Z_{\sigma} \delta_{\mu \rho}-Z_{\rho} \delta_{\mu \sigma}\right)+\frac{\partial}{\partial Z_{\mu}}\left(Z_{\rho} \delta_{\nu \sigma}-Z_{\sigma} \delta_{\nu \rho}\right)\right](1-\kappa) D_{1}\left(-Z^{2}\right)\right\} .
\end{aligned}
$$


Here $Z=X-X^{\prime}, G_{2}$ is the gluon condensate, $D, D_{1}$ are invariant functions normalized to

$$
D(0)=D_{1}(0)=1
$$

and $\kappa$ is a parameter measuring the non-abelian character of the correlator.

Indeed, if we consider an abelian theory we have to replace the gluon field strengths $\mathcal{G}_{\mu \nu}$ by abelian field strengths $\mathcal{F}_{\mu \nu}$, which satisfy the homogenous Maxwell equation

$$
\epsilon_{\mu \nu \rho \sigma} \partial_{\nu} \mathcal{F}_{\rho \sigma}(X)=0
$$

if there are no magnetic monopoles present. It is easy to see that this implies $\kappa=0$ in (2.61). Thus, in an abelian theory, the $D$-term in (2.61) is absent without magnetic monopoles but would be non-zero if the vacuum contained a magnetic monopole condensate. In the non-abelian theory the $D$-term has no reason to vanish. In fact, we will see that it dominates over the $D_{1}$-term. The abelian analogy suggests an interpretation of the $D$-term as being due to an effective chromomagnetic monopole condensate in the QCD vacuum.

Two further assumptions are made in the SVM:

- Ass. 2: The correlation functions $D\left(-Z^{2}\right)$ and $D_{1}\left(-Z^{2}\right)$ fall off rapidly for $Z^{2} \rightarrow \infty$. There exists a characteristic finite correlation length $a$, which we define as

$$
a:=\int_{0}^{\infty} \mathrm{d} Z D\left(-Z^{2}\right) .
$$

A typical ansatz for the function $D$, incorporating assumption 2 , is as follows:

$$
D\left(-Z^{2}\right)=\frac{27}{64} a^{-2} \int \mathrm{d}^{4} K e^{i K Z} K^{2}\left[K^{2}+\left(\frac{3 \pi}{8 a}\right)^{2}\right]^{-4},
$$

which leads to

$$
D\left(-Z^{2}\right) \propto \exp \left(-\frac{3 \pi|Z|}{8 a}\right) \quad \text { for } Z^{2} \rightarrow \infty .
$$

The function $D_{1}$ is chosen such that

$$
\left(4+Z_{\mu} \frac{\partial}{\partial Z_{\mu}}\right) D_{1}\left(-Z^{2}\right)=4 D\left(-Z^{2}\right)
$$


which leads to

$$
D_{1}\left(-Z^{2}\right)=\left(Z^{2}\right)^{-2} \int_{0}^{Z^{2}} \mathrm{~d} v 2 v D(-v) .
$$

With (2.67) the contracted field strength correlator has the form (cf. (2.61):

$$
F_{\mu \nu \mu \nu}=\frac{1}{2} G_{2} D\left(-Z^{2}\right) .
$$

The ansatz (2.65), (2.67) can be compared to a lattice gauge theory calculation of the gluon field strength correlator [44] in order to fix the parameters. One finds (cf. 44, 450 and Fig. 9):

$$
\begin{aligned}
a & =0.35 \mathrm{fm}, \\
\kappa & =0.74, \\
G_{2} & =(496 \mathrm{MeV})^{4}
\end{aligned}
$$

with an educated guess for the error of $\approx 10 \%$. Of course, from Fig. 9 we get only the product $\kappa \cdot G_{2}$. These quantities are obtained separately by measuring on the lattice several components of the correlator (2.60). The value for $G_{2}$ in (2.70) from the lattice calculations is somewhat larger than from phenomenology. This can be understood as follows. The lattice calculations of [44] are for the pure gluon theory. In the real world light quarks are present. Their effect is estimated to reduce the value of $G_{2}$ substantially [46].

We note that the correlation length is smaller, albeit not much smaller than a typical radius $R$ of a light hadron (cf. e.g. [47], [48, [49], [50]):

$$
R \sim 0.7-1 \mathrm{fm} .
$$

Still

$$
a^{2} / R^{2} \approx 0.2-0.3
$$

is a reasonably small number and this will be important for us in the following.

We come now to the third assumption made in the SVM:

- Ass. 3: Factorization of higher point gluon field strength correlators. 
In detail assumption 3 reads as follows (cf. 45):

All expectation values of an odd number of products of shifted field strengths vanish:

$$
\langle\hat{\mathcal{G}}(1) \ldots \hat{\mathcal{G}}(2 n+1)\rangle=0 \quad \text { for } \quad n=0,1,2, \ldots
$$

Here we set as shorthand

$$
\hat{\mathcal{G}}(i) \equiv \hat{\mathcal{G}}_{\alpha_{i} \beta_{i}}^{a_{i}}\left(Y, X_{i} ; C_{X_{i}}\right) .
$$

For even number of shifted field strengths we set in the SVM:

$$
\begin{aligned}
\langle\hat{\mathcal{G}}(1) \ldots \hat{\mathcal{G}}(2 n)\rangle= & \sum_{\substack{\text { all pairings } \\
\left(i_{1}, j_{1}\right) \ldots\left(i_{n}, j_{n}\right)}}\left\langle\hat{\mathcal{G}}\left(i_{1}\right) \hat{G}\left(j_{1}\right)\right\rangle \ldots\left\langle\hat{\mathcal{G}}\left(i_{n}\right) \hat{\mathcal{G}}\left(j_{n}\right)\right\rangle,
\end{aligned}
$$

where $n=1,2, \ldots$.

We note that $\langle\hat{\mathcal{G}}(1)\rangle$ must vanish due to colour conservation since the QCD vacuum has no preferred direction in colour space. The vanishing of the other correlators of odd numbers of field strengths, postulated in (2.73), as well as the factorization property (2.75) are strong dynamical assumptions. They mean that the vacuum fluctuations are assumed to be of the simplest type: a Gaussian random process.

For some applications of the SVM, for instance the calculation of the Wegner-Wilson loop described below, assumption 3 is not crucial and can be relaxed. But for the applications of the SVM to high energy scattering (cf. sect. 3) assumption 3 is crucial. In any case we prefer to specify the model completely, thus giving it maximal predictive power. On the other hand, of course, the model can then more easily run into difficulties in comparison with experiments.

\subsection{The Wegner-Wilson loop in the stochastic vacuum model}

We have now specified the SVM completely and can proceed to show how this model produces confinement. We consider a static quark-antiquark pair at distance $R$ from each other and ask for the potential $V(R)$. To calculate 
$V(R)$ we start with a rectangular Wegner-Wilson loop in the $X_{1}-X_{4}$ plane (Fig. 10): Let $C$ be the loop and $S$ the rectangle, $C=\partial S$. Then

$$
W(C)=\frac{1}{3}\left\langle\operatorname{Tr} \mathrm{P} \exp \left[-i g \int_{C} \mathrm{~d} Z_{\mu} \mathcal{G}_{\mu}(Z)\right]\right\rangle
$$

and

$$
V(R)=-\lim _{T \rightarrow \infty} \frac{1}{T} \ln W(C) .
$$

To evaluate $W(C)$ in the SVM we first transform the line integral of the potentials in (2.76) into a surface integral of field strengths, using the nonabelian version of Stokes theorem (cf. sect. 2.2). For this we choose a reference point $Y$ in $S$. We get then

$$
W(C)=\frac{1}{3}\left\langle\operatorname{Tr} \mathrm{P} \exp \left[-i g \int_{S} \mathrm{~d} X_{1} \mathrm{~d} X_{4} \hat{\mathcal{G}}_{14}\left(Y, X, C_{X}\right)\right]\right\rangle
$$

where $\hat{\mathcal{G}}_{\mu \nu}$ are the field strengths parallel-transported from $X$ to $Y$ along a straight line $C_{X}$. The path-ordered way to integrate over $S$ in a fantype net (cf. Fig. 8) is indicated by $\mathrm{P}$. To evaluate the expectation value of the path-ordered exponential in (2.78), we will use the technique of the cumulant expansion and the assumptions 1-3 of the SVM. We make the following replacements in the formulae (2.38) ff. of sect. 2.3:

$$
\begin{aligned}
& \tau \rightarrow\left(X_{1}, X_{4}\right) \\
& B\left(\tau_{i}\right) \rightarrow \hat{\mathcal{G}}_{14}\left(Y, X^{(i)}, C_{X^{(i)}}\right) \\
& E\left(B\left(\tau_{1}\right) . . B\left(\tau_{n}\right)\right) \rightarrow \frac{1}{3} \operatorname{Tr}\left\langle\hat{\mathcal{G}}_{14}\left(Y, X^{(1)} ; C_{X^{(1)}}\right) \ldots \hat{\mathcal{G}}_{14}\left(Y, X^{(n)} ; C_{X^{(n)}}\right)\right\rangle \\
& t \rightarrow-i g \\
& f(t) \rightarrow W(C) .
\end{aligned}
$$

With this we can express $W(C)$ as an exponential of cumulants as given in (2.41). From Ass. 3 of the SVM we get that all cumulants for odd numbers of gluon field strengths vanish. The lowest nontrivial cumulant is the second one, $K_{2}$, and from (2.51) we find

$$
K_{2}(1,2) \rightarrow \frac{1}{3} \operatorname{Tr}\left\langle\mathrm{P}\left(\hat{\mathcal{G}}_{14}\left(Y, X^{(1)} ; C_{X^{(1)}}\right) \hat{\mathcal{G}}_{14}\left(Y, X^{(2)} ; C_{X^{(2)}}\right)\right\rangle\right.
$$


If we cut off the cumulant expansion (2.41) at $n=2$ we get then for the Wegner-Wilson loop

$$
\begin{aligned}
& W(C)=\exp \left\{-\frac{g^{2}}{2} \int_{S} \mathrm{~d} X_{1} \mathrm{~d} X_{4} \int_{S} \mathrm{~d} X_{1}^{\prime} \mathrm{d} X_{4}^{\prime}\right. \\
& \left.\left\langle\frac{1}{3} \operatorname{Tr} \mathrm{P}\left[\hat{\mathcal{G}}_{14}\left(Y, X, C_{X}\right) \hat{\mathcal{G}}_{14}\left(Y, X^{\prime}, C_{X^{\prime}}\right)\right]\right\rangle\right\} \\
& =\exp \left\{-\frac{2 \pi^{2}}{3} \int_{S} \mathrm{~d} X_{1} \mathrm{~d} X_{4} \int_{S} \mathrm{~d} X_{1}^{\prime} \mathrm{d} X_{4}^{\prime} F_{1414}\left(X-X^{\prime}\right)\right\},
\end{aligned}
$$

where in the last step we used (2.61), i.e. Ass. 1 of the SVM. Next the assumption 2 of short-range correlation for the field strengths enters in a crucial way. This implies that for large Wegner-Wilson loops the integration over $X_{1}^{\prime}, X_{4}^{\prime}$ keeping $X_{1}, X_{4}$ fixed in (2.81) gives essentially a factor $a^{2}$. The remaining $X_{1}, X_{4}$ integration gives then the area of $S=R T$. Thus we arrive at an area law for the Wegner-Wilson loop for $R, T \gg a$ :

$$
W(C)=e^{-\sigma \cdot R \cdot T}
$$

where the constant $\sigma$ is obtained as

$$
\sigma=\frac{\pi^{3} \kappa G_{2}}{36} \int_{0}^{\infty} \mathrm{d} Z^{2} D\left(-Z^{2}\right)=\frac{32 \pi \kappa G_{2} a^{2}}{81} .
$$

We leave the proof of (2.83) as an exercise for the reader. Comparing (2.77) and (2.82) we find that the SVM produces a linearly rising potential

$$
V(R)=\sigma \cdot R \text { for } R \gg a,
$$

where $\sigma$ is the string tension.

The results (2.82)-(2.84) were derived in the framework of the SVM in [40], where the model was introduced, and they are interesting in quite a number of respects:

Only for $\kappa \neq 0$ does one get an area law and thus confinement. The $D_{1}$ term which is the only one present in the abelian theory produces no confinement. In the SVM confinement is related to an effective chromomagnetic monopole condensate in the vacuum.

The short range correlation for the field strengths produces a long range correlation for the potentials if the $D$ term is present in (2.61), i.e. if $\kappa \neq 0$. 
The string tension $\sigma$ is obtained numerically with the input (2.70) as

$$
\sigma=(420 \mathrm{MeV})^{2}
$$

This is very consistent with the phenomenological determination of $\sigma \simeq$ $(430 \mathrm{MeV})^{2}$ from the charmonium spectrum [51].

The SVM has been applied in many other studies of low energy hadronic phenomena (cf. [39] for a review). It was, for instance, possible to calculate flux distributions around a static quark-antiquark pair [52]. The results compare well with lattice gauge theory calculations wherever the latter are available.

Let us come back to the calculation of the Wegner-Wilson loop above. It is legitimate to ask about the contribution of higher cumulants to $W(C)$. How do they modify (2.81)? It turns out that higher cumulants may cause some problems, which we discuss in Appendix A together with a proposal for their remedy.

\section{Soft Hadronic Reactions}

\subsection{General considerations}

In this section we will present a microscopic approach towards hadron-hadron diffractive scattering (cf. [26], [53]). Consider as an example elastic scattering of two hadrons $h_{1}, h_{2}$

$$
h_{1}+h_{2} \rightarrow h_{1}+h_{2}
$$

at high energies and small momentum transfer. We will look at reaction (3.1) from the point of view of an observer living in the "femto-universe", i.e. we imagine having a microscope with resolution much better than $1 \mathrm{fm}$ for observing what happens during the collision. Of course, we should choose an appropriate resolution for our microscope. If we choose the resolution much too good, we will see too many details of the internal structure of the hadrons which are irrelevant for the reaction considered and we will miss the essential features. The same is true if the resolution is too poor. In 53 we used a series of simple arguments based on the uncertainty relation to estimate this appropriate resolution.

Let $t=0$ be the nominal collision time of the hadrons in (3.1) in the c.m. system. This is the time when the hadrons $h_{1,2}$ have maximal spatial 
overlap. Let furthermore be $t_{0} / 2$ the time when, in an inelastic collision, the first produced hadrons appear. We estimate $t_{0} \approx 2 \mathrm{fm}$ from the LUND model of particle production [54]. Then the appropriate resolution, i.e. the cutoff in transverse parton momenta $k_{T}$ of the hadronic wave functions to be chosen for describing reaction (3.1) in an economical way is

$$
k_{T}^{2} \leq \sqrt{s} /\left(2 t_{0}\right)
$$

where $\sqrt{s}$ is the c.m. energy. Modes with higher $k_{T}$ can be assumed to be integrated out. With this we could argue that over the time interval

$$
-\frac{1}{2} t_{0} \leq t \leq \frac{1}{2} t_{0}
$$

the following should hold or better: could be assumed:

(a) The parton state of the hadrons does not change qualitatively, i.e. parton annihilation and production processes can be neglected for this time.

(b) Partons travel in essence on straight lightlike world lines.

(c) The partons undergo "soft" elastic scattering.

The strategy is now to study first soft parton-parton scattering in the femto-universe. There, the relevant interaction will turn out to be mediated by the gluonic vacuum fluctuations. We have argued at length in section 2 that these have a highly nonperturbative character. In this way the nonperturbative QCD vacuum structure will enter the picture for high energy soft hadronic reactions. Once we have solved the problem of parton-parton scattering we have to fold the partonic $S$-matrix with the hadronic wave functions of the appropriate resolution (3.2) to get the hadronic $S$-matrix elements.

We will now give an outline of the various steps in this program.

\subsection{The functional integral approach to parton-parton scattering}

Consider first quark-quark scattering:

$$
q\left(p_{1}\right)+q\left(p_{2}\right) \rightarrow q\left(p_{3}\right)+q\left(p_{4}\right)
$$

where we set 


$$
\begin{aligned}
& s=\left(p_{1}+p_{2}\right)^{2} \\
& t=\left(p_{1}-p_{3}\right)^{2} \\
& u=\left(p_{1}-p_{4}\right)^{2} .
\end{aligned}
$$

Of course, free quarks do not exist in QCD, but let us close our eyes to this at the moment. Now we should calculate the scattering of the quarks over the finite time interval (3.3) of length $t_{0} \approx 2 \mathrm{fm}$. Let us assume that $2 \mathrm{fm}$ is nearly infinitely long on the scale of the femto universe and use the standard reduction formula, due to Lehmann, Symanzik, and Zimmermann, to relate the $S$-matrix element for (3.4) to an integral over the 4-point function of the quark fields. We use the following normalization for our quark states

$$
\begin{aligned}
\left\langle q\left(p_{j}, s_{j}, A_{j}\right)\right| & \left.q\left(p_{k}, s_{k}, A_{k}\right)\right\rangle \\
& =\delta_{s_{j} s_{k}} \delta_{A_{j} A_{k}}(2 \pi)^{3} \sqrt{2 p_{j}^{0} 2 p_{k}^{0}} \delta^{3}\left(\mathbf{p}_{j}-\mathbf{p}_{k}\right) \\
& \equiv \delta(j, k),
\end{aligned}
$$

where $s_{j}, s_{k}$ are the spin and $A_{j}, A_{k}$ the colour indices. With this we get

$$
\begin{aligned}
\left\langle q\left(p_{3}, s_{3}, A_{3}\right) q\left(p_{4}, s_{4}, A_{4}\right)|S| q\left(p_{1}, s_{1}, A_{1}\right) q\left(p_{2}, s_{2}, A_{2}\right)\right\rangle \\
\equiv\langle 3,4|S| 1,2\rangle \\
=\langle 3,4 \mid 1,2\rangle+Z_{\psi}^{-2}\left\{\left(4 \mid\left(i \overrightarrow{\not \partial}-m_{q}^{\prime}\right) \otimes\left(3 \mid\left(i \overrightarrow{\not \partial}-m_{q}^{\prime}\right)\right.\right.\right. \\
\langle 0|\mathrm{~T}(q(4) q(3) \bar{q}(1) \bar{q}(2))| 0\rangle \\
\left.\left.\left.\left(i \overleftarrow{\not \partial}+m_{q}^{\prime}\right) \mid 1\right) \otimes\left(i \overleftarrow{\not \partial}+m_{q}^{\prime}\right) \mid 2\right)\right\} .
\end{aligned}
$$

Here $Z_{\psi}$ is the quark wave function renormalization constant and $m_{q}^{\prime}$ the renormalized quark mass. We use a shorthand notation

$$
\begin{aligned}
& \mid j) \equiv u_{s_{j}, A_{j}}\left(p_{j}\right) e^{-i p_{j} x_{j}}, \\
& \left(j \mid=e^{i p_{j} x_{j}} \bar{u}_{s_{j}, A_{j}}\left(p_{j}\right),\right. \\
& q(j) \equiv q\left(x_{j}\right), \\
& (j=1, . ., 4),
\end{aligned}
$$

where $u$ is the spinor in Dirac and colour space. Two repeated arguments $j, k, \ldots$ imply a space-time integration, for instance

$$
\left.\bar{q}(1)\left(i \overleftarrow{\not \partial}+m_{q}^{\prime}\right) \mid 1\right) \equiv \int \mathrm{d} x_{1} \bar{q}\left(x_{1}\right)\left(i \overleftarrow{\not \partial}+m_{q}^{\prime}\right) e^{-i p_{1} x_{1}} u_{s_{1}, A_{1}}\left(p_{1}\right)
$$


Thus in (3.7) we have four integrations over $x_{1}, \ldots, x_{4}$.

We can represent the 4-point function of the quark fields as a functional integral:

$$
\begin{aligned}
& \langle 0|\mathrm{~T}(q(4) q(3) \bar{q}(1) \bar{q}(2))| 0\rangle \\
& =Z^{-1} \int \mathcal{D}(G, q, \bar{q}) \exp \left\{i \int \mathrm{d} x \mathcal{L}_{\mathrm{QCD}}(x)\right\} q(4) q(3) \bar{q}(1) \bar{q}(2),
\end{aligned}
$$

where $Z$ is the partition function:

$$
Z=\int \mathcal{D}(G, q, \bar{q}) \exp \left\{i \int \mathrm{d} x \mathcal{L}_{\mathrm{QCD}}(x)\right\} .
$$

The QCD Lagrangian (1.1) is bilinear in the quark and antiquark fields. Thus - as is well known - the functional integration over $q$ and $\bar{q}$ can be carried out immediately. After some standard manipulations we arrive at the following expression:

$$
\begin{aligned}
& \langle 0|\mathrm{~T}(q(4) q(3) \bar{q}(1) \bar{q}(2))| 0\rangle \\
& =\frac{1}{Z} \int \mathcal{D}(G) \exp \left\{-i \int \mathrm{d} x \frac{1}{2} \operatorname{Tr}\left(G_{\lambda \rho}(x) G^{\lambda \rho}(x)\right)\right\} \\
& \operatorname{det}\left[-i\left(i \gamma^{\lambda} D_{\lambda}-m_{q}+i \epsilon\right)\right] \\
& \left\{\frac{1}{i} S_{F}(4,2 ; G) \frac{1}{i} S_{F}(3,1 ; G)-(3 \leftrightarrow 4)\right\} .
\end{aligned}
$$

Here $S_{F}(j, k ; G) \equiv S_{F}\left(x_{j}, x_{k} ; G\right)$ is the unrenormalized quark propagator in the given gluon potential $G_{\lambda}(x)$. We have

$$
\left(i \gamma^{\mu} D_{\mu}-m_{q}\right) S_{F}(x, y ; G)=-\delta(x-y) .
$$

Functional integrals as in (3.12) will occur frequently further on. Let $F(G)$ be some functional of the gluon potentials. We will denote the functional integral over $F(G)$ by brackets $\langle F(G)\rangle_{G}$ :

$$
\begin{aligned}
& \langle F(G)\rangle_{G}:=\frac{1}{Z} \int \mathcal{D}(G) \exp \left\{-i \int \mathrm{d} x \frac{1}{2} \operatorname{Tr}\left(G_{\lambda \rho} G^{\lambda \rho}\right)\right\} . \\
& \operatorname{det}\left[-i\left(i \gamma^{\lambda} D_{\lambda}-m_{q}+i \epsilon\right)\right] F(G) .
\end{aligned}
$$

Now we insert (3.12) in (3.7) and get

$$
\langle 3,4|S| 1,2\rangle=\langle 3,4 \mid 1,2\rangle-Z_{\psi}^{-2}\left\langle\mathcal{M}_{31}^{F}(G) \mathcal{M}_{42}^{F}(G)-(3 \leftrightarrow 4)\right\rangle_{G},
$$


where

$$
\begin{aligned}
& \mathcal{M}_{k j}^{F}(G)=\left(k\left|\left(i \overrightarrow{\not \partial}-m_{q}^{\prime}\right) S_{F}\left(i \overleftarrow{\not \not}+m_{q}^{\prime}\right)\right| j\right), \\
& (k=3,4 ; j=1,2) .
\end{aligned}
$$

The term $\mathcal{M}_{31}^{F} \cdot \mathcal{M}_{42}^{F}$ on the r.h.s. of $(3.15)$ corresponds to the $t$-channel exchange diagrams, the second term, where the role of the quarks 3 and 4 is interchanged, to the $u$-channel exchange diagrams (Fig. 11). The latter term should be unimportant for high energy, small $|t|$ scattering. Thus we neglect it in the following. For the scattering of different quark flavours it is absent anyway. We set, therefore:

$$
\langle 3,4|S| 1,2\rangle \cong\langle 3,4 \mid 1,2\rangle-Z_{\psi}^{-2}\left\langle\mathcal{M}_{31}^{F}(G) \mathcal{M}_{42}^{F}(G)\right\rangle_{G}
$$

We can interpret $\mathcal{M}_{k j}^{F}(G)$ as scattering amplitude for quark $j$ going to $k$ in the fixed gluon potential $G_{\lambda}(x)$. To see this, let us define the wave function

$$
\left.\left.\mid \psi_{p_{j}}^{F}\right)=S_{F}\left(i \overleftarrow{\not \partial}+m_{q}^{\prime}\right) \mid j\right)
$$

which satisfies the Dirac equation with the gluon potential $G_{\lambda}(x)$ :

$$
\left.\left(i \gamma^{\lambda} D_{\lambda}-m_{q}\right) \mid \psi_{p_{j}}^{F}\right)=0
$$

Furthermore we use the Lippmann-Schwinger equation for $S_{F}$ :

$$
S_{F}=S_{F}^{(0)}-S_{F}^{(0)}\left(g \notin t-\delta m_{q}\right) S_{F}
$$

where $S_{F}^{(0)}$ is the free quark propagator for mass $m_{q}^{\prime}$ and $\delta m_{q}=m_{q}^{\prime}-m_{q}$ is the quark mass shift. Inserting $(3.20)$ and (3.18) in (3.16) gives after some simple algebra

$$
\mathcal{M}_{k j}^{F}(G)=\left(p_{k}|(g \notin-\delta m)| \psi_{p_{j}}^{F}\right) .
$$

This represents $\mathcal{M}_{k j}^{F}$ in the form a scattering amplitude should have: a complete incoming wave is folded with the potential and the free outgoing wave. However, there is a small problem. The wave function $\mid \psi_{p_{j}}^{F}$ ) defined in (3.18) does not satisfy the boundary condition which we should have for using it in the scattering amplitude, i.e. it does not go to a free incoming wave for time $t \rightarrow-\infty$. The wave function with this boundary condition is obtained by replacing the Feynman propagator $S_{F}$ in (3.18) by the retarded one, $S_{r}$. 
We have shown in [53] that in the high energy limit this replacement can indeed be justified for the calculation of $\mathcal{M}_{k j}^{F}(G)$ if the gluon potential $G_{\lambda}(x)$ contains only a limited range of frequencies:

$$
\mathcal{M}_{k j}^{F}(G) \simeq \mathcal{M}_{k j}^{r}(G)=\left(p_{k}|(g \not t-\delta m)| \psi_{p_{j}}^{r}\right),
$$

where

$$
\left.\left.\mid \psi_{p_{j}}^{r}\right)=S_{r}\left(i \overleftarrow{\not \partial}+m_{q}^{\prime}\right) \mid j\right)
$$

which satisfies

$$
\begin{gathered}
\left.\left(i \gamma^{\mu} D_{\mu}-m_{q}\right) \mid \psi_{p_{j}}^{r}\right)=0, \\
\left.\left.\mid \psi_{p_{j}}^{r}\right) \rightarrow \mid j\right) \text { for } t \rightarrow-\infty .
\end{gathered}
$$

We summarize the results of this subsection: At high energies and small $|t|$ the quark-quark scattering amplitude can be obtained by calculating first the scattering of quark 1 going to 3 and 2 going to 4 in the same fixed gluon potential $G_{\lambda}(x)$. Let the corresponding scattering amplitudes be $\mathcal{M}_{31}^{r}(G)$ and $\mathcal{M}_{42}^{r}(G)$ (cf. (3.22)). Then the product of these two amplitudes is to be integrated over all gluon potentials with the measure given by the functional integral (3.14) and this gives the quark-quark scattering amplitude (3.17). The point of our further strategy is to continue making suitable high energy approximations in the integrand of this functional integral which will be evaluated finally using the methods of the stochastic vacuum model.

In the following it will be convenient to choose a coordinate system for the description of reaction (3.4) where the quarks 1,3 move with high velocity in essence in positive $x^{3}$ direction, the quarks 2,4 in negative $x^{3}$ direction. We define the light cone coordinates

$$
x_{ \pm}=x^{0} \pm x^{3}
$$

and in a similar way the \pm components of any 4 -vector. With this we have for the 4-momenta of our quarks:

$$
p_{j}=\left(\begin{array}{ccc}
\frac{1}{2} p_{j+} & + & \frac{\mathbf{p}_{j T}^{2}+m_{q}^{\prime 2}}{2 p_{j+}} \\
& \mathbf{p}_{j T} & \\
\frac{1}{2} p_{j+} & - & \frac{\mathbf{p}_{j T}^{2}+m_{q^{\prime}}^{2}}{2 p_{j+}}
\end{array}\right)
$$


for $j=1,3$ with $p_{j+} \rightarrow \infty$ and

$$
p_{k}=\left(\begin{array}{ccc}
\frac{1}{2} p_{k-} & + & \frac{\mathbf{p}_{k T}^{2}+m_{q^{\prime}}^{\prime 2}}{2 p_{k-}} \\
& \mathbf{p}_{k T} & \\
-\frac{1}{2} p_{k-} & + & \frac{\mathbf{p}_{k T}^{2}+m_{q^{\prime}}^{2}}{2 p_{k-}}
\end{array}\right)
$$

for $k=2,4$ with $p_{k-} \rightarrow \infty$.

\subsection{The Eikonal expansion}

The problem is now to solve the Dirac equation (3.24) for arbitrary external gluon potential $G_{\lambda}(x)$. Of course, we cannot do this exactly. But we are only interested in the high energy, small $|t|$ limit. This suggests to use an eikonal type approach. This works indeed, but it is not as straightforward as one would think at first, since the Dirac equation is of first order in the derivatives, whereas the eikonal expansion is easy to make for for a secondorder differential equation. What we did in [53 was to make an ansatz for the Dirac field $\psi_{p_{j}}^{r}(x)$ in terms of a "potential" $\phi_{j}(x)$ as follows:

$$
\psi_{p_{j}}^{r}(x)=\left(i \gamma^{\lambda} D_{\lambda}+m_{q}\right) \phi_{j}(x) .
$$

A suitable boundary condition for $\phi_{j}(x)$ which is compatible with (3.25) is

$$
\phi_{j}(x) \rightarrow \frac{1}{p_{j}^{0}+m_{q}} \frac{1+\gamma^{0}}{2} e^{-i p_{j} x} u\left(p_{j}\right)
$$

for $t \rightarrow-\infty$. Inserting (3.29) into the Dirac equation (3.24) gives:

$$
\left\{i \gamma^{\lambda} D_{\lambda}-m_{q}\right\}\left\{i \gamma^{\rho} D_{\rho}+m_{q}\right\} \phi_{j}(x)=0
$$

For the case of no gluon field, $G_{\lambda}(x)=0$, the covariant derivatives $D_{\lambda}$ degenerate to the ordinary ones, $\partial_{\lambda}$, and (3.31) to the Klein-Gordon equation:

$$
\left(\square+m_{q}^{2}\right) \phi_{j}(x)=0 .
$$

Thus the problem for the Dirac field is in essence reduced to a scalar field-type problem which is handled more easily. 
Now it is more or less straightforward to turn the theoretical crank and to obtain the eikonal approximations for $\phi_{j}(x)$ and $\psi_{p_{j}}^{r}(x)$, respectively. Take $j=1$ as an example. We make the ansatz

$$
\phi_{1}(x)=e^{-i p_{1} x} \tilde{\phi}_{1}(x) .
$$

Inserting $p_{1}$ from (3.27) we see that on the r.h.s. of (3.33) the fast varying phase factor is

$$
\exp \left(-i \frac{1}{2} p_{1+} x_{-}\right)
$$

since $p_{1+} \rightarrow \infty$. Assuming all the remaining factors to vary slowly with $x$ we insert (3.33) in (3.31) and order the resulting terms in powers of $1 / p_{1+}$. The solution of (3.31) to leading order in $1 / p_{1+}$ is then easily obtained. The final formula for $\psi_{p_{1}}^{r}(x)$ reads:

$$
\psi_{p_{1}}^{r}(x)=V_{-}\left(x_{+}, x_{-}, \mathbf{x}_{T}\right) \cdot\left\{1+O\left(\frac{1}{p_{1+}}\right)\right\} e^{-i p_{1} x} u\left(p_{1}\right),
$$

where

$$
V_{-}\left(x_{+}, x_{-}, \mathbf{x}_{T}\right)=\mathrm{P}\left\{\exp \left[-\frac{i}{2} g \int_{-\infty}^{x_{+}} \mathrm{d} x_{+}^{\prime} G_{-}\left(x_{+}^{\prime}, x_{-}, \mathbf{x}_{T}\right)\right]\right\}
$$

and $\mathrm{P}$ means path ordering. When coming in, the quark picks up a non-abelian phase factor, just the ordered integral of $G$ along the path. Of course, $V_{-}$ is a connector, as studied in section 2.1, but now in Minkowski space and for a straight light-like line running from $-\infty$ to $x$ (Fig. 12).

In a similar way we obtain for the quark with initial momentum $p_{2}$, i.e. the one coming in from the right, for $p_{2-} \rightarrow \infty$ :

$$
\psi_{p_{2}}^{r}(x)=V_{+}\left(x_{+}, x_{-}, \mathbf{x}_{T}\right)\left\{1+O\left(\frac{1}{p_{2-}}\right)\right\} e^{-i p_{2} x} u\left(p_{2}\right),
$$

where

$$
V_{+}\left(x_{+}, x_{-}, \mathbf{x}_{T}\right)=\mathrm{P}\left\{\exp \left[-\frac{i}{2} g \int_{-\infty}^{x_{-}} \mathrm{d} x_{-}^{\prime} G_{+}\left(x_{+}, x_{-}^{\prime}, \mathbf{x}_{T}\right)\right]\right\} .
$$

Recall that

$$
G_{ \pm}(x)=\left(G^{0 a}(x) \pm G^{3 a}(x)\right) \frac{\lambda_{a}}{2}
$$

are matrices in colour space. Thus path ordering in (3.35) and (3.37) is essential.

A solution for $\psi_{p_{1}}^{r}\left(\psi_{p_{2}}^{r}\right)$ as a series expansion in powers of $1 / p_{1+}\left(1 / p_{2-}\right)$ was obtained to all orders in [55]. 


\subsection{The quark-quark scattering amplitude}

We can now insert our high energy approximations (3.34), (3.36) for $\psi_{p_{1,2}}^{r}(x)$ in the expression for $\mathcal{M}_{k j}^{(r)}$ in (3.22). The resulting integrals are easily done and we get for $p_{1+}, p_{3+} \rightarrow \infty$ :

$$
\begin{aligned}
\mathcal{M}_{31}^{r}(G) \rightarrow & \int \mathrm{d} x e^{i\left(p_{3}-p_{1}\right) x} \\
& \bar{u}\left(p_{3}\right)(g \notin(x)-\delta m) V_{-}(x) u\left(p_{1}\right) \\
\rightarrow & \frac{i}{2} \int \mathrm{d} x_{+} \mathrm{d} x_{-} \mathrm{d}^{2} x_{T} \exp \left[\frac{i}{2}\left(p_{3}-p_{1}\right)_{+} x_{-}-i\left(\mathbf{p}_{3}-\mathbf{p}_{1}\right)_{T} \cdot \mathbf{x}_{T}\right] \\
& \bar{u}\left(p_{3}\right) \gamma_{+} \frac{\partial}{\partial x_{+}} V_{-}\left(x_{+}, x_{-}, \mathbf{x}_{T}\right) u\left(p_{1}\right) \\
\rightarrow & i \sqrt{p_{3+} p_{1+}} \cdot \delta_{s_{3}, s_{1}} \int \mathrm{d} x_{-} \mathrm{d}^{2} x_{T} \\
& \exp \left[\frac{i}{2}\left(p_{3}-p_{1}\right)_{+} x_{-}-i\left(\mathbf{p}_{3}-\mathbf{p}_{1}\right)_{T} \cdot \mathbf{x}_{T}\right] \\
& {\left[V_{-}\left(\infty, x_{-}, \mathbf{x}_{T}\right)-11\right]_{A_{3}, A_{1}} . }
\end{aligned}
$$

In a similar way we obtain for $p_{2-}, p_{4-} \rightarrow \infty$ :

$$
\begin{aligned}
\mathcal{M}_{42}^{r}(G) \rightarrow & i \sqrt{p_{4-} \cdot p_{2-}} \cdot \delta_{s_{4}, s_{2}} \\
& \int \mathrm{d} y_{+} \mathrm{d}^{2} y_{T} \exp \left[\frac{i}{2}\left(p_{4}-p_{2}\right)_{-} y_{+}-i\left(\mathbf{p}_{4}-\mathbf{p}_{2}\right)_{T} \cdot \mathbf{y}_{T}\right] \\
& {\left[V_{+}\left(y_{+}, \infty, \mathbf{y}_{T}\right)-11\right]_{A_{4}, A_{2}} . }
\end{aligned}
$$

Here we have written out the spin and colour indices of the in- and outgoing quarks (cf. (3.6), (3.7)). We have used furthermore:

$$
\begin{aligned}
& \bar{u}_{s_{3}}\left(p_{3}\right) \gamma^{\mu} u_{s_{1}}\left(p_{1}\right) \rightarrow \sqrt{p_{3+} p_{1+}} \cdot \delta_{s_{3}, s_{1}} n_{+}^{\mu} \text { for } \quad p_{1,3+} \rightarrow \infty \\
& \bar{u}_{s_{4}}\left(p_{4}\right) \gamma^{\mu} u_{s_{2}}\left(p_{2}\right) \rightarrow \sqrt{p_{4-} p_{2-}} \cdot \delta_{s_{4}, s_{2}} n_{-}^{\mu} \text { for } \quad p_{2,4-} \rightarrow \infty
\end{aligned}
$$

where

$$
n_{ \pm}^{\mu}=\left(\begin{array}{c}
1 \\
0 \\
0 \\
\pm 1
\end{array}\right)
$$

Finally, the $x_{+}\left(x_{-}\right)$integration for $\mathcal{M}_{31}^{r}\left(\mathcal{M}_{42}^{r}\right)$ could be done with the help of (2.15) or rather the analogous equation for connectors in Minkowski space time. 
Now we can insert everything in our expression (3.17) for the $S$-matrix element. This gives:

$$
\begin{aligned}
& \langle 3,4|S| 1,2\rangle=\langle 3,4 \mid 1,2\rangle+ \\
& \sqrt{p_{3+} p_{1+} p_{4-} p_{2-}} \cdot \delta_{s_{3}, s_{1}} \delta_{s_{4}, s_{2}} Z_{\psi}^{-2} \int \mathrm{d} x_{-} \mathrm{d}^{2} x_{T} \int \mathrm{d} y_{+} \mathrm{d}^{2} y_{T} \\
& \exp \left[\frac{i}{2}\left(p_{3}-p_{1}\right)_{+} x_{-}-i\left(\mathbf{p}_{3}-\mathbf{p}_{1}\right)_{T} \cdot \mathbf{x}_{T}\right] \\
& \exp \left[\frac{i}{2}\left(p_{4}-p_{2}\right)_{-} y_{+}-i\left(\mathbf{p}_{4}-\mathbf{p}_{2}\right)_{T} \cdot \mathbf{y}_{T}\right] \\
& \left\langle\left[V_{-}\left(\infty, x_{-}, \mathbf{x}_{T}\right)-11\right]_{A_{3}, A_{1}}\left[V_{+}\left(y_{+}, \infty, \mathbf{y}_{T}\right)-11\right]_{A_{4}, A_{2}}\right\rangle_{G} .
\end{aligned}
$$

From translational invariance of the functional integral we have:

$$
\begin{aligned}
& \left\langle\left[V_{-}\left(\infty, x_{-}, x_{T}\right)-11\right]_{A_{3}, A_{1}}\left[V_{+}\left(y_{+}, \infty, \mathbf{y}_{T}\right)-11\right]_{A_{4}, A_{2}}\right\rangle_{G} \\
& =\left\langle\left[V_{-}\left(\infty, 0, \mathbf{x}_{T}-\mathbf{y}_{T}\right)-11\right]_{A_{3}, A_{1}}\left[V_{+}(0, \infty, 0)-11\right]_{A_{4}, A_{2}}\right\rangle_{G}
\end{aligned}
$$

Inserting (3.44) in (3.43), we can pull out the $\delta$-function for the overall energymomentum conservation and we get finally:

$$
\begin{aligned}
& \langle 3,4|S| 1,2\rangle=\langle 3,4 \mid 1,2\rangle++i(2 \pi)^{4} \delta\left(p_{3}+p_{4}-p_{1}-p_{2}\right)\langle 3,4|T| 1,2\rangle \\
& \langle 3,4|T| 1,2\rangle=i 2 \sqrt{p_{3+} p_{1+} p_{4-} p_{2-}} \cdot \delta_{s_{3}, s_{1}} \delta_{s_{4}, s_{2}}\left(-Z_{\psi}^{-2}\right) \int \mathrm{d}^{2} z_{T} e^{i \mathbf{q}_{T} \cdot \mathbf{z}_{T}} \\
& \left\langle\left[V_{-}\left(\infty, 0, \mathbf{z}_{T}\right)-11\right]_{A_{3}, A_{1}}\left[V_{+}(0, \infty, 0)-11\right]_{A_{4}, A_{2}}\right\rangle_{G} \cdot
\end{aligned}
$$

Here $q$ is the momentum transfer:

$$
\begin{aligned}
q & =p_{1}-p_{3}=p_{4}-p_{2} \\
q^{2} & =t
\end{aligned}
$$

In the high energy limit $q$ is purely transverse

$$
q \rightarrow\left(\begin{array}{c}
0 \\
\mathbf{q}_{T} \\
0
\end{array}\right), \quad q^{2} \rightarrow-\mathbf{q}_{T}^{2}
$$

Using different techniques the type of formula (3.45) was also obtained in [56]. 
In (3.45) we still have to calculate the wave function renormalization constant $Z_{\psi}$. This can be done by considering a suitable matrix element of the baryon number current

$$
\frac{1}{3} \bar{q}(x) \gamma^{\mu} q(x)
$$

which is conserved and, therefore, needs no renormalization. The result is (cf. [53]):

$$
Z_{\psi}=\frac{1}{3}\left\langle\operatorname{Tr} V_{-}(\infty, 0,0)\right\rangle_{G}
$$

Let us summarize the results obtained so far:

The quark-quark scattering amplitude (3.45) is diagonal in the spin indices. Thus we get helicity conservation in high energy quark-quark scattering. Using (3.41) we can write the spin factor in (3.45) as

$$
2 \sqrt{p_{3+} p_{1+} p_{4-} p_{2-}} \delta_{s_{3}, s_{1}} \delta_{s_{4}, s_{2}} \cong \bar{u}_{s_{3}}\left(p_{3}\right) \gamma^{\mu} u_{s_{1}}\left(p_{1}\right) \bar{u}_{s_{4}}\left(p_{4}\right) \gamma_{\mu} u_{s_{2}}\left(p_{2}\right)
$$

for $p_{1,3+} \rightarrow \infty$ and $p_{2,4-} \rightarrow \infty$. This $\gamma^{\mu} \otimes \gamma_{\mu}$ structure was postulated for high energy quark-quark scattering in the Donnachie-Landshoff model for the "Pomeron" coupling [23]. However, a study of quark-antiquark scattering (cf. [53] and below) reveals that (3.45) does not allow an interpretation in terms of an effective Lorentz vector exchange between the quarks. The amplitude (3.45) has both, charge conjugation $C$ even and odd contributions. The $C$ even part corresponds to the "Pomeron" and this is not a Lorentz-vector exchange, but the coherent sum of spin 2,4,6,.. exchanges (cf. [57], [53]). The $C$-odd part corresponds to the "Odderon" introduced in [58] and this is indeed a Lorentz vector exchange.

The quark-quark scattering amplitude (3.45) is governed by the correlation function of two connectors or string operators $V_{ \pm}$associated with two light-like Wegner-Wilson lines (Fig. 13).

The first numerical evaluations of (3.45) using the methods of the SVM were done in [59]. However, it turned out that quark-quark scattering was calculable from (3.45) for abelian gluons only. Indeed, we are embarked on a program to reproduce in this way the results obtained in 25] in the framework of perturbation theory for high energy scattering [60. For the non-abelian gluons difficulties arose having to do with our neglect of quark confinement. This was really a blessing in disguise and the solution proposed in [59] was to consider directly hadron-hadron scattering, representing the hadrons as 
$q \bar{q}$ and $q q q$ wave packets for mesons and baryons, respectively. We will see below how this is done.

\subsection{The scattering of systems of quarks, antiquarks, and gluons}

Let us consider now the scattering of systems of partons. As an example we study the scattering of two $q \bar{q}$ pairs on each other:

$$
q(1)+\bar{q}\left(1^{\prime}\right)+q(2)+\bar{q}\left(2^{\prime}\right) \rightarrow q(3)+\bar{q}\left(3^{\prime}\right)+q(4)+\bar{q}\left(4^{\prime}\right),
$$

where we set $q(i) \equiv q\left(p_{i}, s_{i}, A_{i}\right), \bar{q}\left(i^{\prime}\right) \equiv \bar{q}\left(p_{i}^{\prime}, s_{i}^{\prime}, A_{i}^{\prime}\right)(i=1, \ldots, 4)$ with $p_{i}, s_{i}, A_{i}\left(p_{i}^{\prime}, s_{i}^{\prime}, A_{i}^{\prime}\right)$ the momentum, spin, and colour labels for quarks (antiquarks). We assume the particles with odd (even) indices $i$ to have very large momentum components in positive (negative) $x^{3}$ direction (Fig. 14), i.e. we assume:

$$
\begin{array}{ll}
p_{i+}, p_{i+}^{\prime} \rightarrow \infty & \text { for } \quad i \text { odd } \\
p_{i-}, p_{i-}^{\prime} \rightarrow \infty & \text { for } \quad i \text { even }
\end{array}
$$

The transverse momenta are assumed to stay limited.

Of course, the reduction formula can also be applied for the reaction (3.50). We have to be careful in keeping disconnected pieces. The further strategy is completely analogous to the one employed in sect. 3.2 for deriving (3.7)-(3.17). As we dropped the $u$-channel exchange diagrams in sect. 3.2, we drop now all terms which are estimated to give a vanishing contribution to high energy small momentum transfer scattering. These terms are characterized by large momenta of order of the c.m. energy $\sqrt{s}$ flowing through gluon lines, leading to suppression factors of order $1 / s$. Keeping only the $t$-channel exchange terms and performing all the steps as done in sections 3.2, 3.3 for quark-quark scattering leads finally to a very simple answer for the $S$-matrix element correponding to reaction (3.50) in the limit (3.51):

$$
\begin{aligned}
& \left\langle 3,3^{\prime}, 4,4^{\prime}|S| 1,1^{\prime}, 2,2^{\prime}\right\rangle \rightarrow \\
& \left\langle\left[\delta(3,1)-i Z_{\psi}^{-1} \mathcal{M}_{31}^{r}(G)\right]\left[\delta\left(3^{\prime}, 1^{\prime}\right)-i Z_{\psi}^{-1} \mathcal{M}_{3^{\prime} 1^{\prime}}^{\prime r}(G)\right]\right. \\
& \left.\quad\left[\delta(4,2)-i Z_{\psi}^{-1} \mathcal{M}_{42}^{r}(G)\right]\left[\delta\left(4^{\prime}, 2^{\prime}\right)-i Z_{\psi}^{-1} \mathcal{M}_{4^{\prime} 2^{\prime}}^{\prime r}(G)\right]\right\rangle_{G} .
\end{aligned}
$$


Here $\delta(i, j)$ is as defined in (3.6), $\mathcal{M}_{31}^{r}(G)$ and $\mathcal{M}_{42}^{r}(G)$ are as in (3.22) and $\mathcal{M}^{\prime r}{ }_{3^{\prime} 1^{\prime}}^{\prime r}(G)$ and $\mathcal{M}_{4^{\prime} 2^{\prime}}^{\prime r}(G)$ are the corresponding amplitudes for the scattering of antiquarks on the gluon potential $G_{\lambda}(x)$. We define

$$
\left(j^{\prime} \mid=\bar{v}_{s_{j}^{\prime}, A_{j}^{\prime}}\left(p_{j}^{\prime}\right) e^{-i p_{j}^{\prime} x},\right.
$$

where $v_{s_{j}^{\prime}, A_{j}^{\prime}}\left(p_{j}^{\prime}\right)$ is the Dirac and colour spinor for the antiquark $\bar{q}\left(j^{\prime}\right)$. We have then with $S_{r}$ the retarded Green's function for quarks in the gluon potential $G_{\lambda}(x)$ :

$$
\mathcal{M}_{k^{\prime} j^{\prime}}^{\prime r}(G)=-\left(j^{\prime}\left|\left(i \vec{\not}-m_{q}^{\prime}\right) S_{r}\left(i \grave{\not \partial}+m_{q}^{\prime}\right)\right| k^{\prime}\right)
$$

where $\left(k^{\prime}, j^{\prime}\right)=\left(3^{\prime}, 1^{\prime}\right),\left(4^{\prime}, 2^{\prime}\right)$.

In the high energy limit (3.51) the scattering amplitudes (3.54) can again be obtained in the eikonal approximation. Indeed, we can just use $C$-invariance to get:

$$
\begin{aligned}
& \mathcal{M}_{3^{\prime} 1^{\prime}}^{\prime r}(G) \rightarrow i \sqrt{p_{3+}^{\prime} p_{1+}^{\prime}} \cdot \delta_{s_{3}^{\prime}, s_{1}^{\prime}} \\
& \int \mathrm{d} x_{-} \mathrm{d}^{2} x_{T} \exp \left[\frac{i}{2}\left(p_{3}^{\prime}-p_{1}^{\prime}\right)_{+} x_{-}-i\left(\mathbf{p}_{3}^{\prime}-\mathbf{p}_{1}^{\prime}\right)_{T} \cdot \mathbf{x}_{T}\right] \\
& {\left[V_{-}^{*}\left(\infty, x_{-}, \mathbf{x}_{T}\right)-11\right]_{A_{3}^{\prime}, A_{1}^{\prime}},} \\
& \mathcal{M}_{4^{\prime} 2^{\prime}}^{\prime r}(G) \rightarrow i \sqrt{p_{4-}^{\prime} p_{2-}^{\prime}} \cdot \delta_{s_{4}^{\prime}, s_{2}^{\prime}} \\
& \int \mathrm{d} y_{+} \mathrm{d}^{2} y_{T} \exp \left[\frac{i}{2}\left(p_{4}^{\prime}-p_{2}^{\prime}\right)_{-} y_{+}-\left(\mathbf{p}_{4}^{\prime}-\mathbf{p}_{2}^{\prime}\right)_{T} \cdot \mathbf{y}_{T}\right] \\
& {\left[V_{+}^{*}\left(y_{+}, \infty, \mathbf{y}_{T}\right)-11\right]_{A_{4}^{\prime}, A_{2}^{\prime}} .}
\end{aligned}
$$

In section 3.1 we have argued that over the time interval (3.3) we can neglect parton production and annihilation processes. For the scattering over such a time interval we should have an effective wave function renormalization constant

$$
Z_{\psi}=1,
$$

since the deviation of $Z_{\psi}$ from 1 is just a measure of the strength of quark splitting processes: $q \rightarrow q+G$ etc. In the calculation of $Z_{\psi}$ in the framework of the SVM to be described below, one finds indeed $Z_{\psi}=1$, showing the consistency of this approach with the simple physical picture of sect. 3.1. 
Anticipating this result we see from (3.6), (3.39), (3.40) and (3.55), (3.56) that in the $S$-matrix element $(3.52)$ the $\delta(k, j)\left(\delta\left(k^{\prime}, j^{\prime}\right)\right)$ terms cancel with the 11 terms in $\mathcal{M}_{k j}^{r}(G)\left(\mathcal{M}_{k^{\prime} j^{\prime}}^{\prime r}(G)\right)$ in the limit (3.51). This leads us to the following simple rules for obtaining the $S$-matrix element in the high energy limit: For the right-moving quark $(1 \rightarrow 3)$ we have to insert the factor:

$$
\begin{aligned}
& \mathcal{S}_{q+}(3,1)=\sqrt{p_{3+} p_{1+}} \cdot \delta_{s_{3}, s_{1}} \int \mathrm{d} x_{-} \mathrm{d}^{2} x_{T} \\
& \exp \left[\frac{i}{2}\left(p_{3}-p_{1}\right)_{+} x_{-}-i\left(\mathbf{p}_{3}-\mathbf{p}_{1}\right)_{T} \cdot \mathbf{x}_{T}\right] \\
& V_{-}\left(\infty, x_{-}, \mathbf{x}_{T}\right)_{A_{3}, A_{1}} .
\end{aligned}
$$

For the right-moving antiquark $\left(1^{\prime} \rightarrow 3^{\prime}\right)$ we have to insert the factor:

$$
\begin{aligned}
& \mathcal{S}_{\bar{q}+}\left(3^{\prime}, 1^{\prime}\right)=\sqrt{p_{3+}^{\prime} p_{1+}^{\prime}} \cdot \delta_{s_{3}^{\prime}, s_{1}^{\prime}} \int \mathrm{d} x_{-} \mathrm{d}^{2} x_{T} \\
& \exp \left[\frac{i}{2}\left(p_{3}^{\prime}-p_{1}^{\prime}\right)_{+} x_{-}-i\left(\mathbf{p}_{3}^{\prime}-\mathbf{p}_{1}^{\prime}\right)_{T} \cdot \mathbf{x}_{T}\right] \\
& V_{-}^{*}\left(\infty, x_{-}, \mathbf{x}_{T}\right)_{A_{3}^{\prime}, A_{1}^{\prime}} .
\end{aligned}
$$

For the left-moving quark $(2 \rightarrow 4)$ and antiquark $\left(2^{\prime} \rightarrow 4^{\prime}\right)$ we have to exchange the + and - labels everywhere in (3.58) and (3.59). This gives:

$$
\begin{aligned}
& \mathcal{S}_{q-}(4,2)=\sqrt{p_{4-} p_{2-}} \cdot \delta_{s_{4}, s_{2}} \int \mathrm{d} y_{+} \mathrm{d}^{2} y_{T} \\
& \exp \left[\frac{i}{2}\left(p_{4}-p_{2}\right)_{-} y_{+}-i\left(\mathbf{p}_{4}-\mathbf{p}_{2}\right)_{T} \cdot \mathbf{y}_{T}\right] \\
& V_{+}\left(y_{+}, \infty, \mathbf{y}_{T}\right)_{A_{4}, A_{2}}, \\
& \mathcal{S}_{\bar{q}-}\left(4^{\prime}, 2^{\prime}\right)=\sqrt{p_{4-}^{\prime} p_{2-}^{\prime}} \cdot \delta_{s_{4}^{\prime}, s_{2}^{\prime}} \int \mathrm{d} y_{+} \mathrm{d}^{2} y_{T} \\
& \exp \left[\frac{i}{2}\left(p_{4}^{\prime}-p_{2}^{\prime}\right)_{-} y_{+}-i\left(\mathbf{p}_{4}^{\prime}-\mathbf{p}_{2}^{\prime}\right)_{T} \cdot \mathbf{y}_{T}\right] \\
& V_{+}^{*}\left(y_{+}, \infty, \mathbf{y}_{T}\right)_{A_{4}^{\prime}, A_{2}^{\prime}} .
\end{aligned}
$$

Finally we have to multiply together the factors $\mathcal{S}_{q^{ \pm}}, \mathcal{S}_{\bar{q}^{ \pm}}$and integrate over all gluon potentials with the functional integral measure (3.14) to get

$$
\left\langle 3,3^{\prime}, 4,4^{\prime}|S| 1,1^{\prime}, 2,2^{\prime}\right\rangle=\left\langle\mathcal{S}_{q+}(3,1) \mathcal{S}_{\bar{q}+}\left(3^{\prime}, 1^{\prime}\right) \mathcal{S}_{q-}(4,2) \mathcal{S}_{\bar{q}-}\left(4^{\prime}, 2^{\prime}\right)\right\rangle_{G}
$$


Going from quarks to antiquarks corresponds, of course, just to the change from the fundamental representation $(3)$ of $S U(3)_{c}$ to the complex conjugate representation $\left(3^{*}\right)$ as we see by comparing (3.58) with (3.59) and (3.60) with (3.61).

It is an easy exercise to show that these rules can be generalized in an obvious way for the scattering of arbitrary systems of quarks and antiquarks on each other. Here we always assume that we have one distinguished collision axis and that one group of partons moves with momenta approaching infinity to the right, the other group to the left. The transverse momenta are assumed to stay limited.

In appendix $\mathrm{B}$ we show that these rules can also be extended to gluons participating in the scattering. We simply have to change the colour representation in (3.58), (3.60) from the fundamental to the adjoint one. In detail we find that for a right-moving gluon

$$
G\left(p_{1}, j_{1}, a_{1}\right) \rightarrow G\left(p_{3}, j_{3}, a_{3}\right)
$$

the following factor has to be inserted in the $S$-matrix element (cf. appendix $\mathrm{B})$ :

$$
\begin{aligned}
& \mathcal{S}_{G+}(3,1)=\sqrt{p_{3+} p_{1+}} \cdot \delta_{j_{3}, j_{1}} \\
& \int \mathrm{d} x_{-} \mathrm{d}^{2} x_{T} \exp \left[\frac{i}{2}\left(p_{3}-p_{1}\right)_{+} x_{-}-i\left(\mathbf{p}_{3}-\mathbf{p}_{1}\right)_{T} \cdot \mathbf{x}_{T}\right] \\
& \mathcal{V}_{-}\left(\infty, x_{-}, \mathbf{x}_{T}\right)_{a_{3}, a_{1}} .
\end{aligned}
$$

Here $j_{1,3}$ are the spin indices which are purely transverse, $1 \leq j_{1,3} \leq 2$. The colour indices are $a_{1}, a_{3}$ with $1 \leq a_{1,3} \leq 8$ and $\mathcal{V}$ is the connector for the adjoint representation of $S U(3)_{c}$ (cf. (B.23)).

For a left-moving gluon we have again to exchange + and - labels in (3.64).

\subsection{The scattering of wave packets of partons representing mesons}

In this section we will go from the parton-parton to hadron-hadron scattering. Our strategy will be to represent hadrons by wave packets of partons, where we make simple "Ansätze" for the wave functions. Then the partonic $S$ matrix element obtained by the rules derived in section 3.5 will be folded 
with these wave functions to give the hadronic $S$-matrix elements. Of course, we always work in the limit of high energies and small momentum transfers.

Let us start by considering meson-meson scattering:

$$
M_{1}\left(P_{1}\right)+M_{2}\left(P_{2}\right) \rightarrow M_{3}\left(P_{3}\right)+M_{4}\left(P_{4}\right),
$$

where $M_{1,3}$ are again the right movers, $M_{2,4}$ the left movers. We make simple "Ansätze" for the mesons as $q \bar{q}$ wave packets as follows:

$$
\begin{aligned}
& \left|M_{j}\left(P_{j}\right)\right\rangle=\int \mathrm{d}^{2} p_{T} \int_{0}^{1} \mathrm{~d} \zeta \frac{1}{(2 \pi)^{3 / 2}} h_{s_{j}, s_{j}^{\prime}}^{j}\left(\zeta, \mathbf{p}_{T}\right) \\
& \frac{1}{\sqrt{3}} \delta_{A_{j}, A_{j}^{\prime}}\left|q\left(p_{j}, s_{j}, A_{j}\right), \bar{q}\left(P_{j}-p_{j}, s_{j}^{\prime}, A_{j}^{\prime}\right)\right\rangle \\
& (j=1, \ldots, 4),
\end{aligned}
$$

where for $j=1,3$ :

$$
\begin{aligned}
& P_{j+} \rightarrow \infty \\
& p_{j+}=\zeta P_{j+}, \\
& \mathbf{p}_{j T}=\frac{1}{2} \mathbf{P}_{j T}+\mathbf{p}_{T}
\end{aligned}
$$

and for $j=2,4$ :

$$
\begin{aligned}
& P_{j-} \rightarrow \infty \\
& p_{j-}=\zeta P_{j-}, \\
& \mathbf{p}_{j T}=\frac{1}{2} \mathbf{P}_{j T}+\mathbf{p}_{T} .
\end{aligned}
$$

Here $\zeta$ is the longitudinal momentum fraction of the quark in the meson, $\mathbf{p}_{T}$ is the relative transverse momentum of $q$ and $\bar{q}$.

We stress that we are not restricting ourselves to spin 0 mesons only. With appropriate functions $h\left(\zeta, \mathbf{p}_{T}\right)$ in (3.66) we can represent states of $q \bar{q}$-mesons of arbitrary spin.

We choose for our states (3.66) the usual continuum normalization:

$$
\left\langle M_{j}\left(P^{\prime}\right) \mid M_{j}(P)\right\rangle=(2 \pi)^{3} 2 P^{0} \delta^{3}\left(\mathbf{P}^{\prime}-\mathbf{P}\right) .
$$

With (3.6) this requires:

$$
\int \mathrm{d}^{2} p_{T} \int_{0}^{1} \mathrm{~d} \zeta 2 \zeta(1-\zeta) h_{s, s^{\prime}}^{* j}\left(\zeta, \mathbf{p}_{T}\right) h_{s, s^{\prime}}^{j}\left(\zeta, \mathbf{p}_{T}\right)=1
$$


In (3.69) and (3.70) no summation over $j$ is to be taken.

For later use we define the wave functions in transverse position space at fixed longitudinal momentum fraction $\zeta$ :

$$
\begin{aligned}
& \varphi_{s, s^{\prime}}^{j}\left(\zeta, \mathbf{x}_{T}\right):=\sqrt{2 \zeta(1-\zeta)} \frac{1}{2 \pi} \int \mathrm{d}^{2} p_{T} \exp \left(i \mathbf{p}_{T} \cdot \mathbf{x}_{T}\right) h_{s, s^{\prime}}^{j}\left(\zeta, \mathbf{p}_{T}\right) \\
& (j=1, \ldots, 4) .
\end{aligned}
$$

With this we define profile functions for the transitions $M_{j} \rightarrow M_{k}$ for right and left movers as:

$$
w_{k, j}\left(\mathbf{x}_{T}\right):=\int_{0}^{1} \mathrm{~d} \zeta\left(\varphi_{s, s^{\prime}}^{k}\left(\zeta, \mathbf{x}_{T}\right)\right)^{*} \varphi_{s, s^{\prime}}^{j}\left(\zeta, \mathbf{x}_{T}\right),
$$

where $k, j$ are both odd or even. Clearly we have (cf. (3.70))

$$
\begin{aligned}
& w_{j, j}\left(\mathbf{x}_{T}\right) \geq 0, \\
& \int \mathrm{d}^{2} x_{T} w_{j, j}\left(\mathbf{x}_{T}\right)=1, \\
& (\text { no summation over } j) .
\end{aligned}
$$

Let us first study the transition of the right movers alone, i.e. the "reaction":

$$
M_{1}\left(P_{1}\right) \rightarrow M_{3}\left(P_{3}\right) .
$$

For stable mesons $M_{1,3}$ we should find that the corresponding $S$-matrix elements are identical to the matrix elements of the unit operator. Is this borne out in our approach?

From the rules given in sect. 3.5 we find easily the $S$-matrix element for the transition

$$
q(1)+\bar{q}\left(1^{\prime}\right) \rightarrow q(3)+\bar{q}\left(3^{\prime}\right)
$$

in the form

$$
\left\langle 3,3^{\prime}|S| 1,1^{\prime}\right\rangle=\left\langle\mathcal{S}_{q+}(3,1) \mathcal{S}_{\bar{q}+}\left(3^{\prime}, 1^{\prime}\right)\right\rangle_{G}
$$

After folding (3.76) with the mesonic wave functions (3.66) we get:

$$
\begin{aligned}
& \left\langle M_{3}\left(P_{3}\right)|S| M_{1}\left(P_{1}\right)\right\rangle=(2 \pi)^{3} 2 P_{1}^{0} \delta^{3}\left(\mathbf{P}_{3}-\mathbf{P}_{1}\right) \\
& \int \mathrm{d}^{2} z_{T} \int_{0}^{1} \mathrm{~d} \zeta_{3} \int_{0}^{1} \mathrm{~d} \zeta_{1} \varphi_{s, s^{\prime}}^{3^{*}}\left(\zeta_{3}, \mathbf{z}_{T}\right) \varphi_{s, s^{\prime}}^{1}\left(\zeta_{1}, \mathbf{z}_{T}\right) \\
& \frac{1}{2} P_{1+} \int \frac{\mathrm{d} z_{-}}{2 \pi} \exp \left[\frac{i}{2} P_{1+}\left(\zeta_{3}-\zeta_{1}\right) z_{-}\right] \\
& \left\langle\frac{1}{3} \operatorname{Tr}\left[V_{-}\left(\infty, z_{-}, \mathbf{z}_{T}\right) V_{-}^{\dagger}(\infty, 0,0)\right]\right\rangle_{G} .
\end{aligned}
$$


Now we remember that we consider the limit $P_{1+} \rightarrow \infty$. Therefore we perform a change of variables in the $z_{-}$integral by setting:

$$
z_{-}^{\prime}:=\frac{1}{2} P_{1+} z_{-} .
$$

This gives:

$$
\begin{aligned}
& \left\langle M_{3}\left(P_{3}\right)|S| M_{1}\left(P_{1}\right)\right\rangle=(2 \pi)^{3} 2 P_{1}^{0} \delta^{3}\left(\mathbf{P}_{3}-\mathbf{P}_{1}\right) \\
& \int \mathrm{d}^{2} z_{T} \int_{0}^{1} \mathrm{~d} \zeta_{3} \int_{0}^{1} \mathrm{~d} \zeta_{1} \varphi_{s, s^{\prime}}^{3^{*}}\left(\zeta_{3}, \mathbf{z}_{T}\right) \varphi_{s, s^{\prime}}^{1}\left(\zeta_{1}, \mathbf{z}_{T}\right) \\
& \int \frac{\mathrm{d} z_{-}^{\prime}}{2 \pi} \exp \left[i\left(\zeta_{3}-\zeta_{1}\right) z_{-}^{\prime}\right] \\
& \left\langle\frac{1}{3} \operatorname{Tr}\left[V_{-}\left(\infty, \frac{2}{P_{1+}} z_{-}^{\prime}, \mathbf{z}_{T}\right) V_{-}^{\dagger}(\infty, 0,0)\right]\right\rangle_{G} \\
& \longrightarrow(2 \pi)^{3} 2 P_{1}^{0} \delta^{3}\left(\mathbf{P}_{3}-\mathbf{P}_{1}\right) \int \mathrm{d}^{2} z_{T} w_{3,1}\left(\mathbf{z}_{T}\right) \\
& \left\langle\frac{1}{3} \operatorname{Tr}\left[V_{-}\left(\infty, 0, \mathbf{z}_{T}\right) V_{-}^{\dagger}(\infty, 0,0)\right]\right\rangle_{G} \text { for } P_{1+} \rightarrow \infty
\end{aligned}
$$

In (3.79) $V_{-}\left(\infty, 0, \mathbf{z}_{T}\right)\left(V_{-}^{\dagger}(\infty, 0,0)\right)$ is the quark (antiquark) connector taken along the line $C_{q}\left(C_{\bar{q}}\right)$, where:

$$
\begin{gathered}
C_{q}: \tau \rightarrow z_{q}(\tau)=\left(\begin{array}{c}
\tau \\
\mathbf{z}_{T} \\
\tau
\end{array}\right), \quad(-\infty<\tau<\infty), \\
C_{\bar{q}}: \tau \rightarrow z_{\bar{q}}(\tau)=\left(\begin{array}{c}
\tau \\
0 \\
\tau
\end{array}\right), \quad(-\infty<\tau<\infty) .
\end{gathered}
$$

But from sect. 2.1 we know that the connector $V_{-}^{\dagger}$ along $C_{\bar{q}}$ is equal to the connector $V_{-}$taken along the oppositely oriented line $\bar{C}_{\bar{q}}$ (cf. (2.25)). Now we will allow ourselves to join the lines $C_{q}$ and $\bar{C}_{\bar{q}}$ at large positive and negative times. We can imagine the gluon potentials to be turned off adiabatically there. We obtain then from the product of the connectors in (3.79) a connector taken along a closed lightlike Wegner-Wilson loop

$$
\frac{1}{3} \operatorname{Tr}\left[V_{-}\left(\infty, 0, \mathbf{z}_{T}\right) V_{-}^{\dagger}(\infty, 0,0)\right] \longrightarrow \mathcal{W}_{+}\left(\frac{1}{2} \mathbf{z}_{T}, \mathbf{z}_{T}\right) .
$$


Here we define

$$
\mathcal{W}_{ \pm}\left(\mathbf{y}_{T}, \mathbf{z}_{T}\right)=\frac{1}{3} \operatorname{TrP} \exp \left[-i g \int_{C_{ \pm}} \mathrm{d} x_{\mu} G^{\mu}(x)\right]
$$

with $C_{+}\left(C_{-}\right)$a lightlike Wegner-Wilson loop in the plane $x_{-}=0\left(x_{+}=0\right)$, where in the transverse space the centre of the loop is at $\mathbf{y}_{T}$ and the vector from the antiquark to the quark line is $\mathbf{z}_{T}$ (Fig. 15).

Inserting now everything in (3.79) we get the simple answer:

$$
\begin{aligned}
& \left\langle M_{3}\left(P_{3}\right)|S| M_{1}\left(P_{1}\right)\right\rangle=(2 \pi)^{3} 2 P_{1}^{0} \delta^{3}\left(\mathbf{P}_{3}-\mathbf{P}_{1}\right) \\
& \int \mathrm{d}^{2} z_{T} w_{3,1}\left(z_{T}\right)\left\langle\mathcal{W}_{+}\left(\frac{1}{2} \mathbf{z}_{T}, \mathbf{z}_{T}\right)\right\rangle_{G} .
\end{aligned}
$$

In the next section we will evaluate the functional integral in (3.84) in the SVM. We will find

$$
\left.\left\langle\mathcal{W}_{+}\left(\mathbf{y}_{T}, \mathbf{z}_{T}\right)\right\rangle_{G}\right|_{S V M}=1
$$

Inserting this in (3.84) leads to the expected result (cf. (3.66)-(3.72)):

$$
\left\langle M_{3}\left(P_{3}\right)|S| M_{1}\left(P_{1}\right)\right\rangle=\left\langle M_{3}\left(P_{3}\right) \mid M_{1}\left(P_{1}\right)\right\rangle .
$$

In our approach the $q \bar{q}$ pair in the right-moving meson $M_{1} \rightarrow M_{3}$ does not interact. Of course this is only valid over our finite time interval (3.3)!

The techniques developed thus far are now easily employed for the reaction (3.65). After performing similar steps as above we arrive at the following $S$-matrix element:

$$
\begin{aligned}
S_{f i}= & \delta_{f i}+i(2 \pi)^{4} \delta\left(P_{3}+P_{4}-P_{1}-P_{2}\right) T_{f i}, \\
T_{f i} \equiv & \left\langle M_{3}\left(P_{3}\right), M_{4}\left(P_{4}\right)|T| M_{1}\left(P_{1}\right), M_{2}\left(P_{2}\right)\right\rangle \\
= & -2 i s \int \mathrm{d}^{2} b_{T} \mathrm{~d}^{2} x_{T} \mathrm{~d}^{2} y_{T} e^{i \mathbf{q}_{T} \cdot \mathbf{b}_{T}} w_{3,1}\left(\mathbf{x}_{T}\right) w_{4,2}\left(\mathbf{y}_{T}\right) \\
& \left\langle\mathcal{W}_{+}\left(\frac{1}{2} \mathbf{b}_{T}, \mathbf{x}_{T}\right) \mathcal{W}_{-}\left(-\frac{1}{2} \mathbf{b}_{T}, \mathbf{y}_{T}\right)-1\right\rangle_{G} .
\end{aligned}
$$

Here $s=\left(P_{1}+P_{2}\right)^{2}$ is the c.m. energy squared and $\mathbf{q}_{T}$ is the momentum transfer, which is purely transverse in the high energy limit:

$$
\mathbf{q}_{T}=\left(\mathbf{P}_{1}-\mathbf{P}_{3}\right)_{T}
$$


From (3.87) we see that the amplitude for soft meson-meson scattering at high energies is governed by the correlation function of two lightlike WegnerWilson loops, where one is in the hyperplane $x_{-}=0$, the other in $x_{+}=$ 0 . The transverse separation between the centres of the two loops is given by $\mathbf{b}_{T}$, the impact parameter. The vectors $\mathbf{x}_{T}$ and $\mathbf{y}_{T}$ give the extensions and orientations of the loops in transverse space (Fig. 16). The loop-loop correlation function has to be integrated over all orientations of the loops in transverse space with the (transition) profile functions of the mesons, $w_{3,1}$ and $w_{4,2}$. Finally a Fourier transform in the impact parameter has to be done.

The methods presented here for meson-meson scattering can of course also be employed for scattering reactions involving baryons and antibaryons. This is sketched in appendix C.

\subsection{The evaluation of scattering amplitudes in the Minkowskian version of the stochastic vacuum model}

In the previous section we have derived expressions for the amplitudes of soft meson-meson scattering at high energies in terms of correlation functions of light-like Wegner-Wilson loops. The task is now to evaluate the corresponding functional integral \langle\rangle$_{G}$ in (3.87). Surely we do not want to make a perturbative expansion there, remembering our argument of sect. 1 (cf. (1.14)). Instead, we will turn to the SVM which did quite well in its applications in Euclidean QCD (sect. 2). Of course, the generalization of the SVM to Minkowski space-time is a bold step which was done in [59], 445]. The authors of these refs. proposed to use in Minkowski space-time just the assumptions 1-3 of the SVM (cf. sect. 2.4), but after having made a suitable analytic continuation. In this way we obtain for instance the Minkowski version of Ass. 1 as (cf. (2.60), (2.61)):

- Ass. 1: The correlator of two field strengths, shifted to a common reference point $y$, is independent of the connecting path and given by:

$$
\begin{aligned}
& \left\langle\frac{g^{2}}{4 \pi^{2}} \hat{G}_{\mu \nu}^{a}\left(y, x ; C_{x}\right) \hat{G}_{\rho \sigma}^{b}\left(y, x^{\prime} ; C_{x^{\prime}}\right)\right\rangle_{G}=\frac{1}{4} \delta^{a b} F_{\mu \nu \rho \sigma}(z), \\
& F_{\mu \nu \rho \sigma}(z)=\frac{1}{24} G_{2}\left\{\left(g_{\mu \rho} g_{\nu \sigma}-g_{\mu \sigma} g_{\nu \rho}\right) \kappa D\left(z^{2}\right)\right.
\end{aligned}
$$




$$
\left.+\frac{1}{2}\left[\frac{\partial}{\partial z^{\nu}}\left(z_{\sigma} g_{\mu \rho}-z_{\rho} g_{\mu \sigma}\right)+\frac{\partial}{\partial z^{\mu}}\left(z_{\rho} g_{\nu \sigma}-z_{\sigma} g_{\nu \rho}\right)\right] \cdot(1-\kappa) D_{1}\left(z^{2}\right)\right\}
$$

Here $z=x-x^{\prime}$ and $D\left(z^{2}\right)$ and $D_{1}\left(z^{2}\right)$ are defined as in (2.65), (2.67) for $z^{2} \leq 0$ and by analytic continuation for $z^{2}>0$.

As a first application let us calculate the expectation value of one lightlike Wegner-Wilson loop. We have from (3.83) using the non-abelian Stokes theorem:

$$
\begin{aligned}
& \left\langle\mathcal{W}_{+}\left(\mathbf{y}_{T}, \mathbf{z}_{T}\right)\right\rangle_{G}=\left\langle\frac{1}{3} \operatorname{TrP} \exp \left[-i g \int_{\mathcal{C}_{+}} \mathrm{d} x_{\mu} G^{\mu}(x)\right]\right\rangle_{G} \\
& =\left\langle\frac{1}{3} \operatorname{Tr} \mathrm{P} \exp \left[-i \frac{g}{2} \int_{S_{+}} \mathrm{d} u \mathrm{~d} v \frac{\partial\left(x^{\mu}, x^{\nu}\right)}{\partial(u, v)} \hat{G}_{\mu \nu}\left(R, x ; C_{x}\right)\right]\right\rangle_{G} .
\end{aligned}
$$

Here $S_{+}$is the (planar) surface spanned into $C_{+}$(Fig. 15) and parametrized by

$$
\begin{aligned}
& x^{\mu}(u, v)=u n_{+}^{\mu}+y^{\mu}-\left(v-\frac{1}{2}\right) z^{\mu}, \\
& -\infty<u<\infty, 0 \leq v \leq 1,
\end{aligned}
$$

where $n_{+}$is as in (3.42) and

$$
y^{\mu}=\left(\begin{array}{c}
0 \\
\mathbf{y}_{T} \\
0
\end{array}\right), \quad z^{\mu}=\left(\begin{array}{c}
0 \\
\mathbf{z}_{T} \\
0
\end{array}\right) .
$$

The reference point on the surface $S_{+}$is denoted by $R$ and $C_{x}$ are straight lines running from $x$ to $R$. From (3.91) we find

$$
\frac{\partial\left(x^{\mu}, x^{\nu}\right)}{\partial(u, v)}=z^{\mu} n_{+}^{\nu}-n_{+}^{\mu} z^{\nu} .
$$

Now we apply the cumulant expansion formulae (cf. sect. 2.3) to (3.90) and use assumptions 1-3 of the SVM. This is completely analogous to the calculations done in sect. 2.5. We get:

$$
\begin{aligned}
\left\langle\mathcal{W}_{+}\left(\mathbf{y}_{T}, \mathbf{z}_{T}\right)\right\rangle_{G}= & \exp \left\{-\frac{\pi^{2}}{6} \int_{S_{+}} \mathrm{d} u \mathrm{~d} v \int_{S_{+}} \mathrm{d} u^{\prime} \mathrm{d} v^{\prime} K_{2}\left(x-x^{\prime}\right)\right. \\
& + \text { higher cumulant terms }\}
\end{aligned}
$$


where

$$
\begin{aligned}
& K_{2}\left(x-x^{\prime}\right)=\frac{\partial\left(x^{\mu}, x^{\nu}\right)}{\partial(u, v)} \frac{\partial\left(x^{\prime \rho}, x^{\prime \sigma}\right)}{\partial\left(u^{\prime}, v^{\prime}\right)} F_{\mu \nu \rho \sigma}\left(x-x^{\prime}\right), \\
& x \equiv x(u, v), \quad x^{\prime} \equiv x^{\prime}\left(u^{\prime}, v^{\prime}\right) .
\end{aligned}
$$

It is an easy exercise to evaluate $K_{2}\left(x-x^{\prime}\right)$ using (3.93) and $F_{\mu \nu \rho \sigma}$ from (3.89). The result is

$$
K_{2}\left(x-x^{\prime}\right)=0
$$

for all $x, x^{\prime} \in S_{+}$. With assumption 3 of the SVM (cf. (2.73)-(2.75)) all higher cumulants are related to the second one. Thus, (3.96) implies also the vanishing of all higher cumulant terms in (3.94) and we get

$$
\left\langle\mathcal{W}_{+}\left(\mathbf{y}_{T}, \mathbf{z}_{T}\right)\right\rangle_{G}=1
$$

A similar argument leads, of course, also to

$$
\left\langle\mathcal{W}_{-}\left(\mathbf{y}_{T}, \mathbf{z}_{T}\right)\right\rangle_{G}=1
$$

In sect. 3.6 we have already used the result (3.97) in the discussion of the transition $M_{1} \rightarrow M_{3}$ to obtain (3.86). We can also use it for the calculation of the wave function renormalization constant $Z_{\psi}$. The expression we obtained for $Z_{\psi}$ in (3.48) can be interpreted as the expectation value of the nonabelian phase factor picked up by a very fast right-moving quark. Now, isolated quarks do not exist. The best approximation for it we can think of is a fast, right-moving quark-antiquark pair with the antiquark being very far away from the quark in transverse direction. In this way we obtain for $Z_{\psi}$ instead of (3.48):

$$
\begin{aligned}
Z_{\psi} & =\lim _{\left|\mathbf{z}_{T}\right| \rightarrow \infty}\left\langle\frac{1}{3} \operatorname{Tr}\left[V_{-}(\infty, 0,0) V_{-}^{\dagger}\left(\infty, 0, \mathbf{z}_{T}\right)\right]\right\rangle_{G} \\
& =\lim _{\left|\mathbf{z}_{T}\right| \rightarrow \infty}\left\langle\mathcal{W}_{+}\left(-\frac{1}{2} \mathbf{z}_{T},-\mathbf{z}_{T}\right)\right\rangle_{G} \\
& =1 .
\end{aligned}
$$

This result was already used in sect. 3.5, (3.57)ff. 
We come now to the evaluation of the loop-loop correlation function of (3.87):

$$
\begin{aligned}
& \left\langle\mathcal{W}_{+}\left(\frac{1}{2} \mathbf{b}_{T}, \mathbf{x}_{T}\right) \mathcal{W}_{-}\left(-\frac{1}{2} \mathbf{b}_{T}, \mathbf{y}_{T}\right)-1\right\rangle_{G} \\
& =\left\langle\left[\mathcal{W}_{+}\left(\frac{1}{2} \mathbf{b}_{T}, \mathbf{x}_{T}\right)-1\right]\left[\mathcal{W}_{-}\left(-\frac{1}{2} \mathbf{b}_{T}, \mathbf{y}_{T}\right)-1\right]\right\rangle_{G} .
\end{aligned}
$$

Here we used (3.97), (3.98). The strategy is as before. We want to transform the line integrals of $\mathcal{W}_{ \pm}$(cf. (3.83)) into surface integrals using the nonabelian Stokes theorem of sect. 2.2. Following the authors of [59], [45] we choose as surface with boundary $C_{+}$and $C_{-}$a double pyramid with apex at the mid-point of $C_{+}$and $C_{-}$which is the origin of our coordinate system (Fig. 17). The mantle of this pyramid is $\mathcal{P}_{+}+\mathcal{P}_{-}$and we have with suitable orientation

$$
\partial\left(\mathcal{P}_{+}+\mathcal{P}_{-}\right)=C_{+}+C_{-} .
$$

In the transverse projection of Fig. 16 the basis surface $S_{+}\left(S_{-}\right)$appears as the line $\bar{q}_{+} q_{+}\left(\bar{q}_{-} q_{-}\right)$and the mantle surface $\mathcal{P}_{+}\left(\mathcal{P}_{-}\right)$as the triangle $0 \bar{q}_{+} q_{+}\left(0 \bar{q}_{-} q_{-}\right)$. From the non-abelian Stokes theorem we obtain now for (3.100):

$$
\begin{aligned}
\langle & {\left.\left[\mathcal{W}_{+}\left(\frac{1}{2} \mathbf{b}_{T}, \mathbf{x}_{T}\right)-1\right]\left[\mathcal{W}_{-}\left(-\frac{1}{2} \mathbf{b}_{T}, \mathbf{y}_{T}\right)-1\right]\right\rangle_{G} } \\
= & \left\langle\left\{\frac{1}{3} \operatorname{Tr} \operatorname{Pexp}\left[-i \frac{g}{2} \int_{\mathcal{P}_{+}} \mathrm{d} u \mathrm{~d} v \frac{\partial\left(x^{\mu}, x^{\nu}\right)}{\partial(u, v)} \hat{G}_{\mu \nu}\left(0, x ; C_{x}\right)\right]-1\right\}\right. \\
& \left.\left\{\frac{1}{3} \operatorname{Tr} \mathrm{P} \exp \left[-i \frac{g}{2} \int_{\mathcal{P}_{-}} \mathrm{d} u^{\prime} \mathrm{d} v^{\prime} \frac{\partial\left(x^{\prime \rho}, x^{\prime \sigma}\right)}{\partial\left(u^{\prime}, v^{\prime}\right)} \hat{G}_{\rho \sigma}\left(0, x^{\prime} ; C_{x^{\prime}}\right)\right]-1\right\}\right\rangle_{G} .
\end{aligned}
$$

So far, it has not been possible to use some version of the cumulant expansion for (3.102). Thus in [59], 45] the path-ordered exponentials on the r.h.s. of (3.102) were expanded directly. The structure of this expansion is as follows:

$$
\begin{aligned}
& \left\langle\left[\mathcal{W}_{+}-1\right]\left[\mathcal{W}_{-}-1\right]\right\rangle_{G} \\
& \sim\left\langle\frac{1}{3} \operatorname{Tr}\left[\int_{\mathcal{P}_{+}} \hat{G}+\int_{\mathcal{P}_{+}} \int_{\mathcal{P}_{+}} \hat{G} \hat{G}+\ldots\right]\right. \\
& \left.\quad \frac{1}{3} \operatorname{Tr}\left[\int_{\mathcal{P}_{-}} \hat{G}+\int_{\mathcal{P}_{-}} \int_{\mathcal{P}_{-}} \hat{G} \hat{G}+\ldots\right]\right\rangle_{G} .
\end{aligned}
$$


The trace of a single shifted field strength vanishes. This means that we cannot exchange a single coloured object in meson-meson scattering (3.65). The first non-trivial contribution in (3.103) comes from the term with two shifted field strengths in each trace. The corresponding correlation function

$$
\left\langle\frac{1}{3} \operatorname{Tr}\left[\int_{\mathcal{P}_{+}} \int_{\mathcal{P}_{+}} \hat{G} \hat{G}\right] \quad \frac{1}{3} \operatorname{Tr}\left[\int_{\mathcal{P}_{-}} \int_{\mathcal{P}_{-}} \hat{G} \hat{G}\right]\right\rangle_{G}
$$

can now be evaluated using the assumptions 1-3 of the Minkowskian version of the SVM. In doing so it is advantageous to transform the surface integrals over the mantles of the pyramids $\mathcal{P}_{ \pm}$into surface integrals over $S_{ \pm}$ and integrals over the volumes $V_{ \pm}$enclosed by $\mathcal{P}_{+}$and $S_{+}$and $\mathcal{P}_{-}$and $S_{-}$, respectively:

$$
\partial\left(V_{ \pm}\right)=\mathcal{P}_{ \pm}-S_{ \pm} .
$$

Then one can use the ordinary Gauss theorem to get simpler integrals.

We will not enter into the details of these calculations here, but only note that the integrations along the directions $x_{+}$and $x_{-}$can easily be done analytically and that one ends up with integrals over the projections of $S_{ \pm}$and $V_{ \pm}$ into the transverse space. Thus, one finally needs the correlator (3.89) for space-like separations only:

$$
\begin{aligned}
& \left(x-x^{\prime}\right)^{2}=z^{2} \leq 0, \\
& x=\left(\begin{array}{c}
0 \\
\mathbf{x}_{T} \\
0
\end{array}\right), \quad x^{\prime}=\left(\begin{array}{c}
0 \\
\mathbf{x}_{T}^{\prime} \\
0
\end{array}\right),
\end{aligned}
$$

where $\mathbf{x}_{T}$ runs over the triangle $\Delta_{+}=0 \bar{q}_{+} q_{+}$and $\mathbf{x}_{T}^{\prime}$ over $\Delta_{-}=0 \bar{q}_{-} q_{-}$in Fig. 16. For space-like separations the correlator functions $D\left(z^{2}\right)$ and $D_{1}\left(z^{2}\right)$ in (3.89) are as in Euclidean space time. The resulting expressions are then of the following structure

$$
\begin{aligned}
& \left\langle\left[\mathcal{W}_{+}-1\right]\left[\mathcal{W}_{-}-1\right]\right\rangle_{G} \sim \\
& \left\{\int_{\Delta_{+}} \mathrm{d}^{2} z_{T} \int_{\Delta_{-}} \mathrm{d}^{2} z_{T}^{\prime} G_{2}\left[. . D\left(-\left(\mathbf{z}_{T}-\mathbf{z}_{T}^{\prime}\right)^{2}\right)+\ldots D_{1}\left(-\left(\mathbf{z}_{T}-\mathbf{z}_{T}^{\prime}\right)^{2}\right)\right]\right\}^{2} .
\end{aligned}
$$

These integrals have to be evaluated numerically. 
With the methods outlined above, we have obtained an (approximate) expression (3.106) for the functional integral \langle\rangle$_{G}$ governing the meson-meson scattering amplitude (3.87). Note that the nonperturbative gluon condensate parameter $G_{2}$ sets the scale in (3.106) and in the integrals to be performed there the vacuum correlation length $a$ enters through the $D$ and $D_{1}$ functions. Thus, on dimensional grounds, we must have in our approximation:

$$
\left\langle\left(\mathcal{W}_{+}-1\right)\left(\mathcal{W}_{-}-1\right)\right\rangle_{G}=G_{2}^{2} a^{8} f\left(\frac{\mathbf{b}_{T}}{a}, \frac{\mathbf{x}_{T}}{a}, \frac{\mathbf{y}_{T}}{a}\right),
$$

where $f$ is a dimensionless function. To obtain the meson-meson scattering amplitude (3.87) we still have to integrate over the profile functions $w_{3,1}\left(\mathbf{x}_{T}\right)$ and $w_{4,2}\left(\mathbf{y}_{T}\right)$. Here the transverse extensions of the mesons - i.e. of the wave packets representing them - enter in the results.

For a detailed exposition of the numerical results obtained in the way sketched above we refer to [45]. Here we only discuss the outcome for protonproton scattering when treated in a similar way (cf. appendix C). A fit to the numerical results gives for the total cross section and the slope parameter at $t=0$ of elastic proton-proton scattering the following representation:

$$
\begin{aligned}
& \sigma_{\text {tot }}(p p)=0.00881\left(\frac{R_{p}}{a}\right)^{3.277} \cdot\left(3 \pi^{2} G_{2}\right)^{2} \cdot a^{10}, \\
& b_{p p}:=\left.\frac{\mathrm{d}}{\mathrm{d} t} \ln \frac{\mathrm{d} \sigma_{e l}}{\mathrm{~d} t}(p p)\right|_{t=0}=1.558 a^{2}+0.454 R_{p}^{2} .
\end{aligned}
$$

Here $R_{p}$ is the proton radius and the formulae (3.108), (3.109) are valid for

$$
1 \leq R_{p} / a \leq 3 .
$$

To compare (3.108), (3.109) with experimental results, we can, for instance, consider the c.m. energy $\sqrt{s}=20 \mathrm{GeV}$ and take as input the following measured values (cf. 445]):

$$
\begin{aligned}
\left.\sigma_{\text {tot }}(p p)\right|_{\text {Pomeron part }} & =35 \mathrm{mb}, \\
b_{p p} & =12.5 \mathrm{GeV}^{-2}, \\
R_{p} & \equiv R_{p, \text { elm }}=0.86 \mathrm{fm} .
\end{aligned}
$$

We obtain then from (3.108) and (3.109):

$$
\begin{aligned}
a & =0.31 \mathrm{fm}, \\
G_{2} & =(507 \mathrm{MeV})^{4} .
\end{aligned}
$$


The values for the correlation length $a$ and for the gluon condensate $G_{2}$ come out in surprising good agreement with the determination of these quantities from the fit to the lattice results (2.70).

But perhaps we were lucky in picking out the right c.m. energy $\sqrt{s}$ and radius for our comparison of theory and experiment. What about the $s$-dependence of the total cross section $\sigma_{t o t}$ and slope parameter $b$ ? The vacuum parameters $G_{2}$ and $a$ should be independent of the energy $\sqrt{s}$. On the other hand, from the discussion in sect. 3.1 leading to (3.2), it seems quite plausible to us that the effective strong interaction radii $R$ of hadrons may depend on $\sqrt{s}$. Let us consider again $p p$ (or $p \bar{p}$ ) elastic scattering. Once we have fixed $G_{2}$ and $a$ from the data at $\sqrt{s}=20 \mathrm{GeV}$ (3.108) and (3.109) give us $\sigma_{\text {tot }}(p p)$ and $b_{p p}$ in terms of the single parameter $R_{p}$, i.e. we obtain as prediction of the model a curve in the plane $b_{p p}$ versus $\sigma_{\text {tot }}(p p)$. This is shown in Fig. 18. It is quite remarkable that the data from $\sqrt{s}=20 \mathrm{GeV}$ up to Tevatron energies, $\sqrt{s}=1.8 \mathrm{TeV}$ follow this curve.

Summarizing this section we can say that explicit calculations for high energy-elastic hadron-hadron scattering near the forward direction have been performed combining the field-theoretic methods of [53 and the Minkowski version of the stochastic vacuum model of [59], [45]. The results are encouraging and support the idea that the vacuum structure of QCD plays an essential role in soft high-energy scattering. We want to point out that these calculations also resolve a possible paradoxon of QCD: On the one hand there are suggestions that the gluon propagator must be highly singular (probably $\propto\left(Q^{2}\right)^{-2}$, cf. e.g. [61]) for momentum transfers $Q^{2} \rightarrow 0$ in order to produce confinement. On the other hand high energy scattering amplitudes are completely regular for $t \rightarrow 0$. A singular gluon propagator will lead in the 2 -gluon exchange model to a singularity for $t=0$ not only for quark-quark scattering but also for hadron-hadron scattering if the latter are considered e.g. as colour dipoles. The resolution of the paradoxon which we can present is intimately connected with the confinement mechanism which we found in the SVM in Sect. 2. The short range correlation of the gluon field strengths governs the $t$-dependence of the hadronic scattering amplitudes and gives rise to their regularity for $t=0$. The gluon propagator on the other hand can be singular for $Q^{2} \rightarrow 0$, since the gluon potentials have a long range correlation as we have seen in Sect. 2. The result (3.108) for the total cross section depends also on the proton radius. This radius dependence does not saturate for large radii in the calculation with non-abelian gluons but does saturate 
in an abelian model [45]. Thus with non-abelian gluons we do not get the additive quark model result [17], and thus not the picture of Donnachie and Landshoff [23], where the "soft Pomeron" couples to individual quarks in the hadrons. The strong radius dependence in (3.108) is due to the $D$-term in the correlator (3.89) which is related to the effective chromomagnetic monopole condensate in the QCD vacuum and which gives rise to the linearly rising quark-antiquark potential, i.e. to string formation (see sect. 2.5). The calculations reported above suggest that in high energy scattering this same term gives rise to a string-string interaction which leads to the radius dependence in (3.108). Note that one does not have to put in the strings by hand. They enter the picture automatically through our lightlike Wegner-Wilson loops. The radius dependence of the cross section occurs, of course, also for mesonbaryon and meson-meson scattering and gives a quantitative understanding of the difference between the $K p$ and $\pi p$ total cross sections and slope parameters at high energies. For this and for further results we refer to [45], [62]. The success of the calculation for $p p(p \bar{p})$ scattering describing correctly the relation of the total cross section versus the slope parameter from $\sqrt{s} \simeq 20$ $\mathrm{GeV}$ up to $\sqrt{s}=1.8 \mathrm{TeV}$ suggests the following simple interpretation: In soft elastic scattering the hadrons act like effective "colour dipoles" with a radius increasing with c.m. energy. The dipole-dipole interaction is governed by the correlation function of two lightlike Wegner-Wilson loops which receives the dominant contribution from the same non-perturbative phenomenon effective chromomagnetic monopole condensation - which leads to string formation and confinement. 


\section{4 "Synchrotron Radiation" from the Vacuum, Electromagnetic Form Factors of Hadrons, and Spin Correlations in the Drell-Yan Reaction}

Let us consider for definiteness again a proton-proton collision at high c.m. energy $\sqrt{s} \gg m_{p}$. We look at this collision in the c.m. system and choose as $x^{3}$-axis the collision axis (Fig. 19). According to Feynman's parton dogma [63] the hadrons look like jets of almost non-interacting partons, i.e. quarks and gluons. Accepting our previous views of the QCD vacuum (Sect. 2), these partons travel in a background chromomagnetic field.

What sort of new effects might we expect to occur in this situation? Consider for instance a quark-antiquark collision in a chromomagnetic field. In our picture this is very similar to an electron-positron collision in a storage ring (Fig. 20). We know that in a storage ring $e^{-}$and $e^{+}$are deflected and emit synchrotron radiation. They also get a transverse polarization due to emission of spin-flip synchrotron radiation [64, 65]. Quite similarly we can expect the quark and antiquark to be deflected by the vacuum fields. Since quarks have electric and colour charge, they should then emit both photon and gluon "synchrotron radiation". Of course, as long as we have quarks within a single, isolated proton (or other hadron) travelling through the vacuum no emission of photons can occur, and we should consider such processes as contributing to the cloud of quasi-real photons surrounding a fast-moving proton. (This is similar in spirit to the well-known WeizsäckerWilliams approximation.) But in a collision process the parent quark or antiquark will be scattered away and the photons of the cloud can become real, manifesting themselves as prompt photons in hadron-hadron collisions.

In ref. [14] we have given an estimate for the rate and the spectrum of such prompt photons using the classical formulae for synchrotron radiation [65]. A more detailed study of soft photon production in hadronic collisions was made in [66]. A sketch of our arguments and calculations is as follows.

In Sect. 2 we discussed the domain picture of the QCD vacuum. In Euclidean space time we have domains in the vacuum of linear size $\simeq a$. Inside one domain the colour fields are highly correlated. The colour field orientations and domain sizes fluctuate, i.e. have a certain distribution. If 
we translate this picture naively to Minkowski space, we arrive at colour correlations there being characterized by invariant distances of order $a$. Then the colour fields at the origin of Minkowski space, for instance, should be highly correlated with the fields in the region

$$
\left|x^{2}\right| \lesssim a^{2}
$$

(cf. Fig. 21). Consider now a fast hadron passing by with one of its quarks going right through $x=0$ on a nearly lightlike world line. It is clear that in such a situation the quark will, from the point of view of the observer, spend a long time in a correlated colour background field. An easy exercise shows furthermore that two quarks of the same hadron will have a very good chance to travel in two different colour domains. The argument is in essence as follows. The quarks have a transverse separation of the order of the hadron radius $R$ whereas the transverse size of a domain is of order $a$ and we have $a^{2} / R^{2} \ll 1$ (cf. (2.72)). Each quark will then wiggle around due to the deflection by the background colour fields in an uncorrelated fashion. This gives us a justification for adding the synchrotron gluon and photon emission of the quarks incoherently. The result we found can be summarized as follows: In the overall c.m. system of the hadron-hadron collision "synchrotron" photons should appear with energies $\omega<300-500$ $\mathrm{MeV}$, i.e. in the very central region of the rapidity space. The number of photons per collision and their spectrum are - apart from logarithms - independent of the c.m. energy $\sqrt{s}$. The dependence of the number of photons on the energy $\omega$ and on the emission angle $\vartheta^{*}$ with respect to the beam axis is obtained as follows for $p N$ collisions:

$$
\frac{\mathrm{d} n_{\gamma}}{\mathrm{d} \omega \mathrm{d} \cos \vartheta^{*}}=\frac{2 \pi \alpha}{\omega^{1 / 3}}\left(l_{\mathrm{eff}}\right)^{2 / 3} \cdot \Sigma\left(\cos \vartheta^{*}\right)
$$

Here $\alpha$ is the fine structure constant and $l_{\text {eff }}$ is the length or time over which the fast quark travelling in the background chromomagnetic field $B_{c}$ obtains by its deflection a transverse momentum of order $\bar{p}_{T} \approx 300 \mathrm{MeV}$, the mean transverse momentum of quarks in a hadron (Fig. 22):

$$
l_{\mathrm{eff}}=\frac{\bar{p}_{T}}{g B_{c}} .
$$

The quantity $\Sigma$ in (4.2) sums up the contributions from all quarks of the initial and final state hadrons. It involves an integration over the quark 
distribution functions of these hadrons. In [66] we found

$$
\Sigma\left(\cos \vartheta^{*}\right) \simeq \frac{0.21}{\left(\sin \vartheta^{*}\right)^{2 / 3}}
$$

for $p N$ collisions at $\sqrt{s}=29 \mathrm{GeV}$.

Our result (4.2) for synchrotron photons should be compared to the innerbremsstrahlungs spectrum

$$
\left.\frac{\mathrm{d} n_{\gamma}}{\mathrm{d} \omega \mathrm{d} \cos \vartheta^{*}}\right|_{\text {bremsstr. }} \propto \frac{1}{\omega \sin ^{2} \vartheta^{*}}
$$

The "synchrotron" radiation from the quarks (4.2) is thus harder than the hadronic bremsstrahlung spectrum. This is welcome, since for $\omega \rightarrow 0$ bremsstrahlung should dominate according to Low's theorem [67].

It is amusing to note that in several experiments an excess of soft prompt photons over the bremsstrahlung calculation has been observed [68]-[72]. The gross features and the order of magnitude of this signal make it a candidate for our "synchrotron" process. A detailed comparison with our formulae has been made in [66] for the results from one experiment [72 with encouraging results. This is shown in Fig. 23 for the $k_{T}$ spectrum of photons at $y=0$, where

$$
\begin{aligned}
& k_{T}=\omega \sin \vartheta^{*} \\
& y=-\ln \tan \left(\vartheta^{*} / 2\right) .
\end{aligned}
$$

We see that the addition of synchrotron photons to the bremsstrahlung ones improves the agreement of theory with the data considerably. We deduce from Fig. $23 l_{\text {eff }} \simeq 20-40 \mathrm{fm}$. Taking $l_{\text {eff }}=20 \mathrm{fm}$ and $\bar{p}_{T}=300 \mathrm{MeV}$, we find for the effective chromomagnetic deflection field from (4.3)

$$
g B_{c}=\frac{\bar{p}_{T}}{l_{\text {eff }}}=(55 \mathrm{MeV})^{2} .
$$

This is much smaller than the vacuum field strength (2.7). Our interpretation of this puzzle is as follows: The colour fields in a fast moving hadron must be shielded. Indeed, a chromomagnetic field of the strength (2.7) would lead to a ridiculously small value for the radius of cyclotron motion of a fast quark. The necessary shielding could be done by gluons in a fast hadron. We know 
from the deep inelastic lepton-nucleon scattering results that a fast nucleon contains many gluons. We may even be brave and turn the argument around: in order for a fast hadron to be able to move through the QCD vacuum, the very strong vacuum chromomagnetic fields must be shielded, making gluons in a fast hadron a necessity. Thus soft photon production in $p p$ collisions may give us a quite unexpected insight into the quark and gluon structure of fast hadrons.

The next topic we want to discuss briefly concerns electromagnetic form factors of hadrons.

We have argued above that the colour fields in the vacuum should give a contribution to the virtual photon cloud of hadrons and we made an estimate of the distribution of these photons using the synchrotron radiation formulae. Consider now any reaction where a quasi-real photon is emitted from a hadron with the hadron staying intact and the photon interacting subsequently. In Fig. 24 we draw the corresponding diagram for a nucleon $N$ :

$$
N(p) \rightarrow N\left(p^{\prime}\right)+\gamma(q) .
$$

The flux of these quasi-real photons is well known. The first calculations in this context are due to Fermi, Weizsäcker, and Williams [73. For us the relevant formula is given in eq. (D.4) of $[74]$. Let $E$ be the energy of the initial nucleon, $G_{E}^{N}\left(Q^{2}\right)$ its electric Sachs formfactor, and let $\omega$ and $q^{2}=-Q^{2}$ be the energy and mass of the virtual photon. Then the distribution of quasi-real photons in the fast-moving nucleon is given by

$$
\mathrm{d} n_{\gamma}^{(\text {excl })}=\frac{\alpha}{\pi} \frac{\mathrm{d} \omega}{\omega} \frac{\mathrm{d} Q^{2}}{Q^{2}}\left[G_{E}^{N}\left(Q^{2}\right)\right]^{2}
$$

where we neglect terms of order $\omega / E$ and $Q^{2} / m_{N}^{2}$ and assume

$$
Q^{2} \gg Q_{\min }^{2} \simeq \frac{m_{N}^{2} \omega^{2}}{E^{2}}
$$

We call (4.9) the exclusive flux since the nucleon stays intact. Now we want to translate (4.9) into a distribution in $\omega$ and the angle $\vartheta^{*}$ of emission of the $\gamma$ (cf. Fig. 24). A simple calculation gives

$$
Q^{2} \simeq \omega^{2} \sin ^{2} \vartheta^{*}
$$




$$
\mathrm{d} n_{\gamma}^{(\mathrm{excl})} \simeq \frac{2 \alpha}{\pi} \frac{\mathrm{d} \omega}{\omega} \frac{\mathrm{d} \vartheta^{*}}{\sin \vartheta^{*}}\left[G_{E}^{N}\left(\omega^{2} \sin ^{2} \vartheta^{*}\right)\right]^{2}
$$

Now we made an "exclusive-inclusive connection" argument in [66]: We require $\mathrm{d} n_{\gamma}^{(\text {excl) }} / \mathrm{d} \omega \mathrm{d} \cos \vartheta^{*}$ to behave as $\omega^{-1 / 3}$ for fixed $\vartheta^{*}$ as we found in (4.2). This implies for the form factor $G_{E}^{N}\left(Q^{2}\right)$ a behaviour as $\left(Q^{2}\right)^{1 / 6}$.

Thus we arrive at the following conclusion: The proton form factor $G_{E}^{p}$ should contain in addition to a "normal" piece connected with the total charge and the hadronic bremsstrahlung in inelastic collisions a piece $\propto\left(Q^{2}\right)^{1 / 6}$ connected with "synchrotron" radiation from the QCD vacuum. For the neutron which has total charge zero we would expect the "normal" piece in $G_{E}^{n}$ to be quite small and the "anomalous" piece to be quite important for not too large $Q^{2}$. Thus the neutron electric formfactor should be an interesting quantity to look for "anomalous" effects $\propto\left(Q^{2}\right)^{1 / 6}$.

In Fig. 25 we show the data on the electric formfactor of the neutron from [75, 76]. We superimpose the curve

$$
G_{(\text {syn })}^{n}\left(Q^{2}\right)=3.6 \cdot 10^{-2}\left(\frac{Q^{2}}{5 \mathrm{fm}^{-2}}\right)^{1 / 6}
$$

which is normalized to the data at $Q^{2}=5 \mathrm{fm}^{-2}$. We see that except in the very low $Q^{2}$ region we get a decent description of the data. For $Q^{2} \rightarrow 0(4.13)$ has to break down since $G_{E}^{n}\left(Q^{2}\right)$ is regular at $Q^{2}=0$. Indeed one knows the slope of $G_{E}^{n}\left(Q^{2}\right)$ for $Q^{2}=0$ from the scattering of thermal neutrons on electrons ([77] and references cited therein):

$$
\left.\frac{\mathrm{d} G_{E}^{n}\left(Q^{2}\right)}{\mathrm{d} Q^{2}}\right|_{Q^{2}=0}=0.019 \mathrm{fm}^{2} .
$$

We see from Fig. 25 that the behaviour of $G_{E}^{n}\left(Q^{2}\right)$ has to change rather quickly as we go away from $Q^{2}=0$. We will now make a simple ansatz which takes into account that $G_{E}^{n}\left(Q^{2}\right)$ can have singularities in the complex $Q^{2}$-plane only for

$$
-\infty<Q^{2} \leq-4 m_{\pi}^{2}
$$

where $m_{\pi}$ is the pion mass. We require a $\left(Q^{2}\right)^{1 / 6}$ behaviour for positive $Q^{2}$ and take the slope of $G_{E}^{n}\left(Q^{2}\right)$ at $Q^{2}=0$ from experiment (4.14). This leads us to the following functional form for $G_{E}^{n}\left(Q^{2}\right)$ :

$$
G_{E}^{n}\left(Q^{2}\right)=0.019 \mathrm{fm}^{2} \cdot Q^{2}\left[1+\frac{Q^{2}}{4 m_{\pi}^{2}}\right]^{-5 / 6} .
$$


It is amusing to see that this gives a decent description of the data (Fig. 25).

What about the electric form factor of the proton $G_{E}^{p}\left(Q^{2}\right)$ ? Here, clearly, we have a dominant "normal" piece connected with the total charge. We will assume that this normal contribution is represented by the usual dipole formula

$$
\begin{aligned}
G_{D}\left(Q^{2}\right) & =\left(1+\frac{Q^{2}}{m_{D}^{2}}\right)^{-2}, \\
m_{D}^{2} & =18.23 \mathrm{fm}^{-2} \hat{=} 0.710 \mathrm{GeV}^{2}
\end{aligned}
$$

which gives a good representation of the data for $Q^{2}=2-4 \mathrm{GeV}^{2}$ [78]. Let us add to this an anomalous piece for smaller $Q^{2}$, connected with synchrotron radiation, and let us assume that this is a purely isovector contribution, consistent with the singularity at $Q^{2}=-4 m_{\pi}^{2}$ in (4.15). We obtain then from (4.15) the following ansatz for $G_{E}^{p}\left(Q^{2}\right)$ :

$$
G_{E}^{p}\left(Q^{2}\right)=G_{D}\left(Q^{2}\right)-G_{E}^{n}\left(Q^{2}\right)=G_{D}\left(Q^{2}\right)\left(1-\Delta\left(Q^{2}\right)\right),
$$

where

$$
\Delta\left(Q^{2}\right)=0.019 \mathrm{fm}^{2} \cdot Q^{2}\left(1+\frac{Q^{2}}{4 m_{\pi}^{2}}\right)^{-5 / 6} \cdot\left(1+\frac{Q^{2}}{m_{D}^{2}}\right)^{2} .
$$

We predict a deviation of the ratio $G_{E}^{p}\left(Q^{2}\right) / G_{D}\left(Q^{2}\right)$ from unity for small $Q^{2}$. It is again amusing to note that such a deviation is indeed observed experimentally [47, 48. Our ansatz does even quite well quantitatively (Fig. 26). For the electromagnetic radius of the proton we predict from (4.17)

$$
\begin{aligned}
\left\langle r_{E}^{2}\right\rangle: & =-\left.6 \frac{\mathrm{d} G_{E}^{p}\left(Q^{2}\right)}{\mathrm{d} Q^{2}}\right|_{Q^{2}=0} \\
& =\frac{12}{m_{D}^{2}}+6 \cdot 0.019 \mathrm{fm}^{2} \\
& =(0.88 \mathrm{fm})^{2} .
\end{aligned}
$$

This checks well with the experimental values quoted in 47]:

$$
\begin{aligned}
\left\langle r_{E}^{2}\right\rangle^{1 / 2}= & 0.88 \pm 0.03 \mathrm{fm}, \text { or } \\
& 0.92 \pm 0.03 \mathrm{fm},
\end{aligned}
$$

depending on the fit used for $G_{E}^{p}\left(Q^{2}\right)$ at low $Q^{2}$. 
To conclude this brief discussion of nucleon form factors, we can summarize our picture as follows: The quarks in the nucleon make a cyclotron-type motion in the chromomagnetic vacuum field. This leads to a spreading out of the charge distribution of the neutron

$$
\rho_{n}(\mathbf{x}) \propto|\mathbf{x}|^{-10 / 3}
$$

corresponding to $G_{E}^{n}\left(Q^{2}\right) \propto\left(Q^{2}\right)^{1 / 6}$. The same effect should lead to a deviation of the proton form factor $G_{E}^{p}\left(Q^{2}\right)$ from the dipole formula for small $Q^{2}$. Concerning the sign of $G_{E}^{n}\left(Q^{2}\right)$ we can in essence follow the arguments put forward in 779 .

One might think — maybe rightly — that these ideas are a little crazy. But we have also worked out some consequences of them for the Drell-Yan reaction (1.9), which make us optimistic. In the lowest order parton process contributing there, we have a quark-antiquark annihilation giving a virtual photon $\gamma^{*}$, which decays then into a lepton pair (Fig. 1):

$$
q+\bar{q} \rightarrow \gamma^{*} \rightarrow \ell^{+} \ell^{-}
$$

In the usual theoretical framework $q$ and $\bar{q}$ are assumed to be uncorrelated and unpolarized in spin and colour if the original hadrons are unpolarized. From our point of view we expect a different situation. Let us go back to Fig. 21 where we sketched the world line of a quark of one fast hadron in a colour domain of extension $\left|x^{2}\right| \lesssim a^{2}$. Let the quark $q$ and antiquark $\bar{q}$ in (4.22) annihilate at the point $x=0$. Here $q$ and $\bar{q}$ come from two different hadrons $h_{1}, h_{2}$ moving with nearly light-like velocity in opposite directions. It is clear that in this situation $q$ and $\bar{q}$ will spend a long time in a highly correlated colour background field (Fig. 27). In [14 we speculated that this may lead to a correlated transverse spin and colour spin polarization of $q$ and $\bar{q}$ due to the chromomagnetic Sokolov-Ternov effect [64, 65]. In [80] we worked out this idea in more detail and found that a transverse $q \bar{q}$ spin correlation influences the $\ell^{+} \ell^{-}$angular distribution in a profound way. Then our colleague H. J. Pirner pointed out to us that data which may be relevant in this connection existed already [81]. And very obligingly these data show a large deviation from the standard perturbative QCD prediction. On the other hand, we can nicely understand the data in terms of our spin correlations and thus vacuum effects in high energy collisions. For more details we refer to [80]. If such spin correlations are confirmed by experiments at higher energies, we would 
presumably have to reconsider the fundamental factorization hypothesis for hard reactions which we sketched in Sect. 1 and which is discussed in detail in [11].

\section{Conclusions}

In these lectures we have discussed various ideas connected with non-perturbative QCD and in particular with the QCD vacuum structure. In Sect. 2 we introduced connectors, the non-abelian Stokes theorem and the cumulant expansion. Then we presented the domain picture of the QCD vacuum and the stochastic vacuum model (SVM). The latter is consistent with the view of the QCD vacuum acting like a dual superconductor: We found that in the SVM the vacuum contains an effective chromomagnetic monopole condensate, whose effect is parametrized by the $D$-term in the gluon field strength correlator (2.61). With these tools we could calculate the expectation value of the Wegner-Wilson loop in the SVM. We found a linearly rising potential between a heavy quark-antiquark pair, $Q \bar{Q}$. This is related to the formation of a "string", a chromoelectric flux tube between $Q \bar{Q}$ as can be seen explicitly in the SVM [52]. Thus in this framework confinement is an effect of the nontrivial QCD vacuum structure. All calculations in sect. 2 were done in Euclidean space time.

In appendix A we discuss some problems which arise when one considers higher cumulant terms in the SVM. We propose as remedy for these problems to do the calculation of the expectation value of the Wegner-Wilson loop in an iterative way, summing step by step over the contributions of various plaquettes. This also leads us to a proposal for including dynamical fermions in the calculation with the expected result that the linearly rising potential levels off and goes to a constant at large $Q \bar{Q}$ separation, where, of course, we have then mesons $Q \bar{q}, q \bar{Q}$ formed of a heavy quark $Q$ (antiquark $\bar{Q}$ ) and a light antiquark $\bar{q}$ (quark $q$ ).

In sections 3,4 we looked at various possible effects of the nontrivial vacuum structure in the Minkowski world. In section 3 we gave a detailed account of a field-theoretic method for the calculation of scattering amplitudes of high-energy soft hadronic reactions. We started from the functional integral and made high energy approximations in the integrand. This led us to give general rules for writing down scattering amplitudes for high energy 
soft reactions at the parton level. Then we considered as representation for hadrons wave packets of partons. The corresponding scattering amplitudes for (quasi-)elastic hadron-hadron scattering were found to be governed by the correlation functions of lightlike Wegner-Wilson loops. The evaluation of these correlation functions was possible with the help of the Minkowski version of the stochastic vacuum model. The comparison with experiment gave very good consistency, supporting the view that the QCD vacuum structure plays an essential role in high energy soft reactions. The framework developed in these lectures can be applied directly to elastic and diffractive hadron-hadron scattering at high energies. In principle we should also be able to apply it to non-diffractive reactions, fragmentation processes etc., but this remains to be worked out.

In sect. 4 we argued that some more startling QCD vacuum effects in high energy collisions may be the appearance of anomalous soft photons in hadron-hadron collisions due to "synchrotron radiation" and spin correlations in the Drell-Yan reaction due to the chromodynamic Sokolov-Ternov effect. Furthermore, we gave an argument that electromagnetic form factors at small $Q^{2}$ should reflect the vacuum structure. Finally we would like to mention that in 82] the rapidity gap events observed at HERA are quantitatively described in terms of the parton model but invoking again nonperturbative QCD effects, possibly connected with the vacuum structure. Another place where the QCD vacuum structure may show up is in certain correlations of hadrons in $Z^{0}$ decays to two jets [14, 83] for which there is also some experimental evidence [84].

We hope to have convinced the reader that the non-perturbative structure of the QCD vacuum is useful in order to understand confinement of heavy quarks. In our view this vacuum structure manifests itself also in high energy soft and hard reactions. We think it is very worth-while to study such effects both from the theoretical and the experimental point of view.

\section{Acknowledgements}

The author is grateful to the organizers of the 1996 Schladming winter school headed by $\mathrm{H}$. Latal for the invitation to lecture there. As basis for the present article the author could use lectures - partly collected by U. Grandel - given at the Workshop on Topics in Field Theory, Graduiertenkolleg Erlangen- 
Regensburg, Banz (1993). The author also thanks the organizers of that meeting, especially F. Lenz, for the invitation to lecture there. The present article is the contribution of the author to the proceedings of both meetings. For many fruitful discussions on topics of these lecture notes the author extends his gratitude to A. Brandenburg, W. Buchmüller, H. G. Dosch, U. Ellwanger, D. Gromes, P. Haberl, P. V. Landshoff, P. Lepage, H. Leutwyler, Th. Mannel, E. Mirkes, H. J. Pirner, M. Rueter, Yu. A. Simonov, G. Sterman, and W. Wetzel. Special thanks are due to H. G. Dosch for a critical reading of the manuscript and to E. Berger and P. Haberl for their help in the preparation of the manuscript. Finally the author thanks Mrs. U. Einecke for her excellent typing of the article.

\section{Appendix A:}

\section{Higher cumulant terms and dynamic fermions in the calculation of the Wegner-Wilson loop in the stochastic vacuum model}

In this appendix we discuss first some problems arising in the calculation of the Wegner-Wilson loop in the SVM (cf. sect. 2.5) if higher cumulants are taken into account. Then we outline a possible remedy which may also point to a way of including the effects of dynamical fermions in the SVM. We start with the replacements (2.79) which allow us to use the cumulant expansion (2.41) for calculating $W(C)$. The second cumulant is given in (2.80):

$$
K_{2}(1,2)=\frac{4}{3} \frac{\pi^{2}}{g^{2}} \mathcal{F}(1,2),
$$

where we set with $F_{\mu \nu \rho \sigma}$ as defined in (2.61):

$$
\begin{aligned}
& \mathcal{F}(i, j) \equiv F_{1414}\left(X^{(i)}-X^{(j)}\right), \\
& F_{1414}(Z)=\frac{G_{2}}{24}\left[\kappa D\left(-Z^{2}\right)\right. \\
& \left.+\frac{1}{2}\left(\frac{\partial}{\partial Z_{1}} Z_{1}+\frac{\partial}{\partial Z_{4}} Z_{4}\right)(1-\kappa) D_{1}\left(-Z^{2}\right)\right], \\
& Z^{2}=Z_{1}^{2}+Z_{4}^{2} .
\end{aligned}
$$


With the assumptions 1-3 of the SVM (sect. 2.4), the next nonvanishing cumulant is $K_{4}$, for which we obtain from (2.47), (2.60), (2.73), (2.75):

$$
K_{4}(1,2,3,4)=-2\left(\frac{\pi^{2}}{g^{2}}\right)^{2}[\mathcal{F}(1,3) \mathcal{F}(2,4) \Theta(1,2,3,4)+\text { perm. }] .
$$

Here $\Theta(1,2,3,4)=1$ if $X^{(1)}>X^{(2)}>X^{(3)}>X^{(4)}$ in the sense of the pathordering on the surface $S$ (cf. Figs. 8, 10) and $\Theta(1,2,3,4)=0$ otherwise.

We start now again from the expression (2.78) for the expectation value of the Wegner-Wilson loop and use the cumulant expansion (2.41) with the identifications (2.79). We get then:

$$
W(C)=\exp \left\{\sum_{n=1}^{\infty} \frac{(-i g)^{n}}{n !} \int_{S_{1}} \ldots \int_{S_{n}} K_{n}(1, \ldots, n)\right\},
$$

where

$$
\int_{S_{i}} \equiv \int_{S} \mathrm{~d} X_{1}^{(i)} \mathrm{d} X_{4}^{(i)}
$$

In the SVM as formulated in sect. 2.4 the cumulants for odd $n$ vanish (cf. Ass. 3). The lowest contribution in (A.5) is then from $n=2$, the next from $n=4$. Cutting off the infinite sum in (A.5) at $n=4$, we get with (A.1) and (A.4):

$$
\begin{aligned}
W(C)= & \exp \left\{-\frac{g^{2}}{2 !} \int_{S_{1}} \int_{S_{2}} K_{2}(1,2)\right. \\
& \left.+\frac{\left(g^{2}\right)^{2}}{4 !} \int_{S_{1}} \cdots \int_{S_{4}} K_{4}(1,2,3,4)\right\} \\
= & \exp \left\{-\frac{1}{2 !} \frac{4 \pi^{2}}{3} \int_{S_{1}} \int_{S_{2}} \mathcal{F}(1,2)\right. \\
& \left.-\frac{1}{4 !} 2 \pi^{4} \int_{S_{1}} \ldots \int_{S_{4}}[\mathcal{F}(1,3) \mathcal{F}(2,4) \Theta(1,2,3,4)+\text { perm. }]\right\} \text { A.7. }
\end{aligned}
$$

Consider now the contribution of the second cumulant in (A.7) for a large Wegner-Wilson loop (Fig. A1 with $R, T \gg a$ ):

$$
I_{2}=\int_{S_{1}} \int_{S_{2}} \mathcal{F}(1,2)
$$


For fixed integration point (1) on $S$ the integration over (2) will give significant contributions only for a region of radius $\sim a$ around $(1)$ since the functions $D\left(-Z^{2}\right)$ and $D_{1}\left(-Z^{2}\right)$, where $Z=\left(X^{(1)}-X^{(2)}\right)$, are assumed to fall off rapidly with increasing $Z^{2}$. From (A.3) we see that the $D_{1}$-term contributes as a total divergence in $\mathcal{F}(1,2)$. Thus we can apply Gauss' law in 2 dimensions for it to transform it to an integral over the boundary $\partial S=C$. In this way we find a contribution from the $D_{1}$ term of order $a^{4} / R^{2}, a^{4} / R T, a^{4} / T^{2}$. From the $D$-term in (A.3) we get a factor $\propto a^{2}$ from the integration over (2) in (A.8). Then the integration over (1) is unconstrained and gives a factor proportional to the whole area of $S$ :

$$
I_{2} \propto R T a^{2}+O\left(a^{4}, a^{4} \frac{T}{R}, a^{4} \frac{R}{T}\right) .
$$

Putting in all factors from (A.3) and using the explicit form (2.65) for the function $D\left(-Z^{2}\right)$ gives for $T \rightarrow \infty$ and $R \gg a$ the result:

$$
\frac{1}{2 !} \frac{4 \pi^{2}}{3} I_{2}=R T \sigma
$$

with $\sigma$ as given in (2.83).

We turn next to the contribution of the 4th cumulant in (A.7) and study the integral

$$
I_{4}=\int_{S_{1}} \ldots \int_{S_{4}} \mathcal{F}(1,3) \mathcal{F}(2,4) \Theta(1,2,3,4) .
$$

Consider again $R, T \gg a$ and fix the integration point (1) (Fig. A2). Then the short-range correlation of the field strengths requires (3) and (1) as well as (4) and (2) to be near to each other, i.e. at a distance $\lesssim a$. The function $\Theta(1,2,3,4)$ requires $(1)>(2)>(3)>(4)$ in the sense of the path ordering on the surface $S$. Using the fan-type net as indicated in Fig. 8 for the application of the non-abelian Stokes theorem with straight line handles from the points $X^{(i)}$ in the surface to the reference point $Y$ we see the following. The pathordering function $\Theta(1,2,3,4)$ restricts (2) to be in the hatched sector of $S$ in Fig. A2. In general this does not restrict (2) to a region close to (1). On the contrary, (2) can vary freely over a triangle of area $\gtrsim a . L$, where $L$ is of order $R$ or $T$ or in between. Thus, the integration over (2) in (A.11) will give at least a factor $\propto(a T)$ if the hatched triangle has its long sides in $X_{4}$ direction (cf. Fig. A2). The integrations over (3) and (4) in (A.11) should 
give factors of $a^{2}$ each. The integration over (1) will give a factor RT. Thus we estimate

$$
I_{4} \propto R T . a^{4} . a T \propto T^{2} .
$$

This is very unpleasant. The 4 th cumulant gives a contribution which domi$\underline{\text { nates }}$ over the one from the second cumulant for $T \rightarrow \infty$. There is no finite limit for $T \rightarrow \infty$ in the expression (2.77). The quark-antiquark potential comes out infinite.

This problem was recognized in ref. [52 and eliminated by hand making an additional assumption: that all but the leading powers in the quotient of the correlation length to the extension parameters $R, T$ of the loop could be neglected. Another simple cure of the problem would be to postulate instead of Ass. 3 (cf. (2.73)-(2.75)) a behaviour of the higher point correlation functions of the shifted gluon field strengths which gives precisely zero for the cumulants $K_{n}$ with $n>2$ in (A.5). In our opinion this would be an unsatisfactory solution, since the model would then only work for a particular fine-tuned set of correlation functions whereas generically the above problem would remain.

We think that the origin of these difficulties in the SVM is the assumption 1 , which states that the correlator should be independent of the reference point $Y$ for arbitrary $Y$. But why should the field strength correlation (2.60) (cf. Fig. 5) be the same if we use a straight line path $C_{X}+\bar{C}_{X^{\prime}}$ to connect $X$ and $X^{\prime}$ or a path which runs on a loop behind the moon? We will replace Ass. 1 by a milder one:

- Ass. 1': $F_{\mu \nu \rho \sigma}$ in (2.60) is independent of the reference point $Y$ and of the curves $C_{X}$ and $C_{X^{\prime}}$ if the reference point $Y$ is close to $X$ and $X^{\prime}$ :

$$
|X-Y| \lesssim a^{\prime},\left|X^{\prime}-Y\right| \lesssim a^{\prime}
$$

where $a^{\prime}$ is of order of $a$.

Now we try to calculate the expectation value of the Wegner-Wilson loop (2.76) using only this weaker hypothesis. We start from the rectangle $S$ of area $R T$ and insert a smaller rectangular loop $C_{1}$ with sides $R-2 a^{\prime}, T-2 a^{\prime}$. On $C_{1}$ we choose $N_{1}$ reference points $Y_{1}, \ldots, Y_{N_{1}}$ and we partition the area between $C$ and $C_{1}$ in $N_{1}$ plaquettes $P_{1}, \ldots, P_{N_{1}}$ of size $\sim a^{\prime 2}$ (Fig. A3). We can now apply the non-abelian Stokes theorem. We start from $Y_{0}$ on $C$ and 
construct a path equivalent to $C$ in the following way: From $Y_{0}$ to $Y_{1}$, then in a fan-type net over the plaquette $P_{1}$ with $Y_{1}$ as reference point, etc., until we arrive at $Y_{N_{1}} \equiv Y_{1}$ from where the path runs back to $Y_{0}$. According to (2.36) we get then:

$$
W(C)=\frac{1}{3} \operatorname{Tr}\left\langle V_{0, N_{1}} V\left(P_{N_{1}}\right) V_{N_{1}, N_{1}-1 \ldots} V_{2,1} V\left(P_{1}\right) V_{1,0}\right\rangle,
$$

where

$$
V\left(P_{j}\right)=\mathrm{P} \exp \left[-i g \int_{P_{j}} \hat{G}_{14}\left(Y_{j}, X^{(j)} ; C_{X^{(j)}}\right)\right]
$$

and $V_{i, j}$ are the connectors from $Y_{j}$ to $Y_{i}$ on straight lines.

- Ass. 4: Now we will make a mean field-type approximation and replace the path-ordered integrals over the plaquettes $P_{j}$ by the corresponding vacuum expectation values:

$$
V\left(P_{j}\right) \rightarrow\left\langle V\left(P_{j}\right)\right\rangle \cdot 11
$$

This is similar in spirit to the "block spin" transformations considered in [85]. For the r.h.s. of (A.15) we can use the cumulant expansion and assumptions 1', 2 and 3 of the SVM. Here the reference point $Y_{j}$ is never too far away from $X^{(j)}$. We get with 11 the unit matrix in colour space

$$
\begin{aligned}
& \left\langle V\left(P_{j}\right)\right\rangle=11 \cdot \exp \left\{\sum_{n=1}^{\infty} \frac{(-i g)^{n}}{n !} \int_{P_{j, 1}} \ldots \int_{P_{j, n}} K_{n}(1, \ldots, n)\right\} \\
& =11 \cdot \exp \left\{-\left|P_{j}\right| \sigma\left[1+O\left(\frac{1}{4 !} \sigma \cdot a \cdot a^{\prime}\right)\right]\right\} .
\end{aligned}
$$

Here we cut off the cumulant expansion at $n=4$ and use the results and estimates (A.7)-(A.12), but now for each plaquette $P_{j}$ instead of the whole surface $S$. We see that the correction terms from the 4 th cumulant are now of manageable size since (cf. (2.70), (2.85)

$$
\sigma a a^{\prime}=\left(\frac{a^{\prime}}{a}\right) \sigma a^{2}=0.56\left(\frac{a^{\prime}}{a}\right)=O(1) .
$$

Then (hopefully) the factorials $1 / n$ ! in the cumulant expansion will lead to small corrections from higher cumulants. 
We can now insert (A.15), (A.16) in A.13) and get

$$
\begin{aligned}
& W(C) \simeq \frac{1}{3} \operatorname{Tr}\left\langle V_{0, N_{1}}\left\langle V\left(P_{N_{1}}\right)\right\rangle V_{N_{1}, N_{1}-1} \ldots V_{2,1}\left\langle V\left(P_{1}\right)\right\rangle V_{1,0}\right\rangle \\
& =\frac{1}{3} \operatorname{Tr}\left\langle V_{0, N_{1}} V_{N_{1}, N_{1}-1} \ldots V_{2,1} V_{1,0}\right\rangle \cdot \exp \left\{-\sum_{j=1}^{N_{1}}\left|P_{j}\right| \sigma\left[1+O\left(\frac{\sigma a a^{\prime}}{4 !}\right)\right]\right\} \\
& =\frac{1}{3} \operatorname{Tr}\left\langle V_{N_{1}, N_{1}-1} \ldots V_{2,1}\right\rangle \exp \left\{-\left(|S|-\left|S_{1}\right|\right) \sigma\left[1+O\left(\frac{\sigma a a^{\prime}}{4 !}\right)\right]\right\} .
\end{aligned}
$$

Here we used also the cyclicity of the trace and $V_{1,0} V_{0, N_{1}}=11$ (cf. (2.24) ). The product of the remaining connectors in (A.18) gives just the Wegner-Wilson loop of the curve $C_{1}=\partial S_{1}$ in Fig. A.3 and we obtain:

$$
W(C)=W\left(C_{1}\right) \exp \left\{-\left(|S|-\left|S_{1}\right|\right) \sigma\left[1+O\left(\frac{\sigma a a^{\prime}}{4 !}\right)\right]\right\} .
$$

The procedure can easily be repeated by inscribing a rectangle $S_{2}$ with boundary $C_{2}=\partial S_{2}$ in $S_{1}$ etc. The final result of this iterative procedure obviously is:

$$
W(C)=\exp \left\{-|S| \sigma\left[1+O\left(\frac{\sigma a a^{\prime}}{4 !}\right)\right]\right\} .
$$

From this we deduce for the "true" string tension in the SVM:

$$
\sigma_{\text {true }}=\sigma\left[1+O\left(\frac{\sigma a a^{\prime}}{4 !}\right)\right]=\sigma[1+\text { terms of order } 5 \%]
$$

where $\sigma$ is given in (2.83). Thus we have justified the result for the string tension in section 2.5, where we used the second cumulant only. We have now relied on the assumption 1' and avoided transportation of field strengths to far away reference points $Y$. The fourth cumulant is estimated to give only a correction of a few percent and the contribution of the 6th, 8th, etc. cumulants can be estimated in an analogous way to be even smaller? Most important, we have avoided the unpleasant result (A.12).

We will now discuss another question which can be raised in connection with the calculation of $W(C)$ in the SVM. Why should we span a minimal

\footnotetext{
${ }^{2}$ Numerical studies suggest that $a^{\prime} \approx 3 a$ should be large enough for obtaining the area law for the plaquettes $P_{j}$ in (A.16) (H. G. Dosch, private communication). We obtain then $\sigma a a^{\prime} \simeq 1.7$ and still correction terms $\lesssim 10 \%$ in A.21.
} 
surface $S$ into the loop $C$ and not use some other, more complicated surface $S^{\prime}$ with the same boundary, $\partial S^{\prime}=C$ ? From the point of view of applying the non-abelian Stokes theorem (2.37) any wiggly surface $S^{\prime}$ would be as good as the flat rectangle $S$ which is the surface of minimal area. However, from the point of view of the iterative calculation with the plaquettes, as explained in this appendix, an arbitrary surface $S^{\prime}$ is clearly not equivalent to $S$. In (A.15) we made the approximation of replacing $V\left(P_{j}\right)\left(j=1, \ldots, N_{1}\right)$ by its vacuum expectation value. This should be a good approximation if the various plaquettes are well separated. Indeed, we would expect that then we can perform the functional integral over the variables related to the regions in Euclidean space time where the various plaquettes are located in a separate and independent way. If, however, two plaquettes overlap or are very near to each other, the above approximation will break down. The point is now that on the minimal surface $S$ neighbouring plaquettes will be at maximal distance from each other. For some arbitrary surface $S^{\prime}$ with wiggles we will inevitably find plaquettes closer together which will make our approximation worse. This gives us some rationale to use a minimal surface $S$ in the applications of the non-abelian Stokes theorem in the framework of the SVM.

So far our calculations should apply to QCD with dynamical gluons and static quarks only. The quark-antiquark potential $V(R)$ in (2.84) rises linearly for $R \rightarrow \infty$. In real life we have, of course, dynamic quarks. If we separate a heavy quark-antiquark pair $Q \bar{Q}$ starting from small $R$ we will first see a linear potential as in (2.84) but at some point the heavy quark $Q$ (antiquark $\bar{Q}$ ) will pick up a light antiquark $\bar{q}$ (quark $q$ ) from the vacuum and form a meson $Q \bar{q}(\bar{Q} q)$. The two mesons can escape to infinity, i.e. the force between them vanishes, the potential $V(R)$ must go to a constant as $R \rightarrow \infty$ :

$$
V(R) \rightarrow V_{\infty} \text { for } \quad R \rightarrow \infty .
$$

Can we understand also this feature of nature in an extension of the SVM?

Let us go back to the approximation (A.15), where we have replaced the integral over the plaquette $P_{j}$ by its vacuum expectation value. More generally we can argue that $V\left(P_{j}\right)$ as defined in (A.14) is an object transforming under a gauge transformation (2.17) as follows (cf. (2.20)):

$$
V\left(P_{j}\right) \rightarrow U\left(Y_{j}\right) V\left(P_{j}\right) U^{-1}\left(Y_{j}\right) .
$$


Any approximation we make should respect this gauge property and indeed the replacement (A.15) does. Now we can ask for a generalisation of (A.15) in the presence of dynamic light quarks. We have then the quark and antiquark variables at the point $Y_{j}$ at our disposal and can construct from them the object

$$
q\left(Y_{j}\right) \bar{q}\left(Y_{j}\right)
$$

which has the gauge transformation property (A.23) and is also rotationally invariant. Let us consider only $u$ and $d$ quarks. Then we suggest as generalization of (A.15) the following replacement:

$$
V_{A B}\left(P_{j}\right) \rightarrow\left\langle V\left(P_{j}\right)\right\rangle_{0} \delta_{A B}-w\left(P_{j}\right) q_{A, f, \alpha}\left(Y_{j}\right) \bar{q}_{B, f, \alpha}\left(Y_{j}\right)
$$

where $A, B$ are the colour indices, $f=u, d$ is the flavour index and $\alpha$ the Dirac index. Furthermore, $\left\langle V\left(P_{j}\right)\right\rangle_{0}$ is as in (A.15), (A.16), and $w\left(P_{j}\right)$ is a coefficient depending on the size of the plaquette $P_{j}$. It can be thought of as representing the chance of producing a $q \bar{q}$ pair from the vacuum gluon fields over the plaquette $P_{j}$. (This is inspired by the discussions of particle production in the LUND model [54]). On dimensional grounds $w\left(P_{j}\right)$ must be proportional to a volume, thus we will set

$$
w\left(P_{j}\right)=c\left|P_{j}\right| \cdot a,
$$

where $c$ is a constant. The idea is that $q \bar{q}$ "production" should feel the gluon fields in a disc of area $\left|P_{j}\right|$ and thickness $a$.

We can now insert the ansatz (A.25) in (A.13). The resulting expression for $W(C)$ is of the form:

$$
\begin{aligned}
W(C) \cong & \frac{1}{3} \operatorname{Tr}\left\langleV _ { 0 , N _ { 1 } } \left[\left\langle V\left(P_{N_{1}}\right)\right\rangle\right.\right. \\
& \left.-w\left(P_{N_{1}}\right) q\left(Y_{N_{1}}\right) \bar{q}\left(Y_{N_{1}}\right)\right] V_{N_{1}, N_{1}-1} \\
& \left.\ldots V_{2,1}\left[\left\langle V\left(P_{1}\right)\right\rangle-w\left(P_{1}\right) q\left(Y_{1}\right) \bar{q}\left(Y_{1}\right)\right] \cdot V_{1,0}\right\rangle .
\end{aligned}
$$

If we mutliply out these brackets we get terms where we have again the Wegner-Wilson loop along $C_{1}$ (Fig. A.3) but then also terms where quarks and antiquarks at different points $Y_{k}, Y_{l}$ are connected. For two neighbouring points, for instance, $k=l+1, l$ this would read:

$$
\ldots q\left(Y_{l+1}\right)\left[-w(l) \bar{q}\left(Y_{l+1}\right) V_{l+1, l} q\left(Y_{l}\right)\right] \bar{q}\left(Y_{l}\right) \ldots
$$


The importance of these terms will increase with increasing $w\left(P_{j}\right)$. Starting from (A.27) we can now inscribe plaquettes into the rectangle $S_{1}$ and transport everything, including the quark variables $q\left(Y_{k}\right) \bar{q}\left(Y_{k}\right)\left(k=1, \ldots, N_{1}\right)$ to a curve $C_{2}$ etc.

To get an orientation we will now make a drastic approximation: For a loop with $R \ll R_{c}$, where $R_{c}$ is some critical value to be determined, we neglect the dynamical quarks, saying that the $w\left(P_{j}\right)$ factors will still be too

small. The result for $W(C)$ and $V(R)$ in the range $a \lesssim R \ll R_{c}$ will then be as in (A.20):

$$
\begin{aligned}
& W(C)=\exp (-R T \sigma), \\
& V(R)=\sigma R, \\
& \left(a \lesssim R \ll R_{c}\right),
\end{aligned}
$$

where we set $\sigma_{\text {true }} \cong \sigma$.

In the other limiting case, $R \gg R_{c}$, we can expect the $q \bar{q}$ terms in (A.27) to dominate. Indeed, let us start with the loop $C$ with sides $R$ and $T$ of Fig. A.3 and inscribe first plaquettes $P_{1, \ldots}, P_{N_{1}}$ of size $a^{\prime 2}$. With the replacement (A.25) this gives for $W(C)$ the expression (A.27). Now we inscribe plaquettes of size $a^{\prime 2}$ in the loop $C_{1}$ and parallel transport everything to a smaller loop $C_{2}$. The new element is that we will now have to parallel-transport also the quark variables, but this poses no problem. We will again obtain an expression like the one shown in (A.27) but now for the curve $C_{2}$ and with increased weight factors $w$ in front of the $q \bar{q}$ terms. We assume that we continue this procedure. Finally, we will obtain an expression like (A.27) but with the terms involving the quark variables at the reference points $Y_{j}$ dominating the expression. Thus we set:

$$
W(C) \cong \frac{1}{3} \operatorname{Tr}\left\langle[-w(N) q(N) \bar{q}(N)] V_{N, N-1} \ldots[-w(1) q(1) \bar{q}(1)] V_{1, N}\right\rangle,
$$

where the points $1, \ldots, N$ are on a curve $C^{\prime}$ of sides $R^{\prime}, T^{\prime}$. This summing up of quark contributions from various plaquettes of size $a^{\prime 2}$ should be reasonable as long as the quarks are inside an area corresponding to their own correlation length, $a_{\chi}$, the chiral correlation length. This can be estimated from the behaviour of the non-local $\bar{q} q$ condensate [86-88] for which one typically makes an ansatz of the form $(q=u, d)$ :

$$
\left\langle\bar{q}\left(X_{2}\right) V_{2,1} q\left(X_{1}\right)\right\rangle=\langle\bar{q} q\rangle e^{-\left|X_{2}-X_{1}\right|^{2} / a_{\chi}^{2}}
$$


Here $\langle\bar{q} q\rangle$ is the local quark condensate for which we set, neglecting isospin breaking (cf. [39):

$$
\langle\bar{q} q\rangle=\frac{1}{2}\langle\bar{u} u+\bar{d} d\rangle=-(0.23 \mathrm{GeV})^{3} .
$$

For the correlation length $a_{\chi}$ one estimates (cf. [88]):

$$
a_{\chi} \cong 1 \mathrm{fm}
$$

It will be reasonable to choose the reference points $1, \ldots, N$ on $C^{\prime}$ also such that the distance between neighbouring points is $a_{\chi}$. Then

$$
\begin{aligned}
R^{\prime} & =R-2 a_{\chi}, \\
T^{\prime} & =T-2 a_{\chi}, \\
N & =\frac{2 T^{\prime}+2 R^{\prime}}{a_{\chi}}=\frac{2(T+R)}{a_{\chi}}-8 .
\end{aligned}
$$

We will now estimate $W(C)$ from $(\widehat{A .29})$ as product of the expectation values of the nonlocal $\bar{q} q$ condensate. We set

$$
w(1)=w(2) \ldots=w(N)=c \cdot a a_{\chi}^{2},
$$

where $c$ should be a numerical constant of order 1 . Furthermore we have from $(\mathrm{A.30})$

$$
\begin{aligned}
& \left\langle\bar{q}_{A, f, \alpha}(j)\left(V_{j, j-1}\right)_{A B} q_{B, f^{\prime}, \alpha^{\prime}}(j-1)\right\rangle \\
& =\frac{1}{4} \delta_{f, f^{\prime}} \delta_{\alpha, \alpha^{\prime}}\langle\bar{q} q\rangle \exp \left[-\frac{\left|X_{j}-X_{j-1}\right|^{2}}{a_{\chi}^{2}}\right] \\
& =\frac{1}{4 e} \delta_{f, f^{\prime}} \delta_{\alpha, \alpha^{\prime}}\langle\bar{q} q\rangle
\end{aligned}
$$

for $\left|X_{j}-X_{j-1}\right|=a_{\chi}$. This gives:

$$
\begin{aligned}
W(C) \cong & -\frac{1}{3} \cdot\left\{\left[-w(N)\left\langle\bar{q}_{f_{1}, \alpha_{1}}(1) V_{1, N} q_{f_{N}, \alpha_{N}}(N)\right\rangle\right]\right. \\
& \left.\ldots\left[-w(1)\left\langle\bar{q}_{f_{2}, \alpha_{2}}(2) V_{2,1} q_{f_{1}, \alpha_{1}}(1)\right\rangle\right]\right\} \\
= & -\frac{8}{3}\left[-\frac{w(1)\langle\bar{q} q\rangle}{4 e}\right]^{N} \\
= & -\frac{8}{3}\left[-\frac{w(1)\langle\bar{q} q\rangle}{4 e}\right]^{\left[\frac{2}{a_{\chi}}(T+R)-8\right]} .
\end{aligned}
$$


For the potential this leads to

$$
V(R)=-\lim _{T \rightarrow \infty} \frac{1}{T} \ln W(C)=\frac{2}{a_{\chi}} \ln \frac{4 e}{[-w(1)\langle\bar{q} q\rangle]} \equiv V_{\infty} .
$$

Thus we find indeed a constant potential for $R \rightarrow \infty$.

For intermediate values of $R$ the potential should change smoothly from the linear rise to the constant behaviour. As a crude approximation we will assume the potential to be continuous but turn abruptly from one to the other behaviour at a critical length $R=R_{c}$ which we define as

$$
R_{c}=V_{\infty} / \sigma
$$

Our simple ansatz for $V(R)$ is then as follows (cf. Fig. A.4):

$$
V(R)=\left\{\begin{array}{cc}
\sigma R & \text { for } \quad R \leq R_{c} \\
V_{\infty}=\sigma R_{c} & \text { for } \quad R \geq R_{c} .
\end{array}\right.
$$

How does the numerics work out taking (A.37) and A.38 seriously? We can estimate the constant $V_{\infty}$ crudely from the mass difference of two $B$-mesons and the $\Upsilon$ resonance

$$
V_{\infty} \cong 2 m_{B}-m_{\Upsilon} \cong 1.1 \mathrm{GeV}
$$

With $\sigma$ from (2.85) we find for $R_{c}$ (A.38):

$$
R_{c} \cong 1,2 \mathrm{fm} \text {. }
$$

A much more elaborate estimate of the string-breaking distance gives 89 $R_{c} \leq 1,6$ to $2,1 \mathrm{fm}$. The lattice calculations of 90 suggest an even larger value for $R_{c}$. From (A.40) and (A.37), (A.32) we get for $w(1)$ and $c$ :

$$
\begin{aligned}
w(1) & =-\frac{4 \exp \left[1-\frac{1}{2} a_{\chi} V_{\infty}\right]}{\langle\bar{q} q\rangle}=0.42 \mathrm{fm}^{3}, \\
c & =\frac{w(1)}{a_{\chi}^{2} a}=1.2 .
\end{aligned}
$$

Thus the probability factor $w(1)$ comes out as we estimated it on geometrical grounds in (A.26).

With these remarks we close this appendix. Of course, much more work is needed in order to decide if our simple ansatz for incorporating dynmical fermions in the stochastic vacuum model is viable or not. 


\section{Appendix B: The scattering of gluons}

In this appendix we will discuss the contribution of gluons to the scattering amplitude of a general parton reaction, generalizing (3.50):

$$
G(1)+G(2)+\ldots+q(k)+\bar{q}\left(k^{\prime}\right)+\ldots \rightarrow G(3)+G(4)+\ldots+q(l)+\bar{q}\left(l^{\prime}\right)+\ldots
$$

Here, as in all of sect. 3, we use the convention that partons with odd (even) number are moving fast in positive (negative) $x^{3}$-direction. Consider thus the transition of a right-moving gluon:

$$
G(1) \equiv G\left(P_{1}, \boldsymbol{\epsilon}_{1}, a_{1}\right) \rightarrow G(3) \equiv G\left(P_{3}, \boldsymbol{\epsilon}_{3}, a_{3}\right),
$$

where $a_{1,3}\left(1 \leq a_{1,3} \leq 8\right)$ are the colour indices, $P_{1}, P_{3}$ are the momenta with $P_{1+}, P_{3+} \rightarrow \infty$, and $\epsilon_{1,3}$ are the polarization vectors which satisfy:

$$
\begin{aligned}
& \mathbf{P}_{1} \boldsymbol{\epsilon}_{1}=0, \\
& \mathbf{P}_{3} \boldsymbol{\epsilon}_{3}=0 \\
& \left|\boldsymbol{\epsilon}_{1}\right|=\left|\boldsymbol{\epsilon}_{3}\right|=1 .
\end{aligned}
$$

In the high energy limit the vectors $\boldsymbol{\epsilon}_{1,3}$ are transverse with corrections of order $\left|\mathbf{p}_{T 1,3}\right| / P_{+1,3}$ which we can neglect. We will argue that such a gluon in a high energy soft reaction is equivalent to a quark-antiquark pair with the same quantum numbers in the limit of the $q$ and $\bar{q}$ being so close to each other in position space that their separation cannot be resolved in the collision. From the contribution of such a $q \bar{q}$ pair to the scattering amplitude we will get in the above limit the gluon contribution.

We start by constructing wave packets similar to the mesonic wave packets of 3.66$)$

$$
\begin{aligned}
& \left|q \bar{q} ; P_{j}, \boldsymbol{\epsilon}_{j}, a_{j}\right\rangle=\int \mathrm{d}^{2} p_{T} \int_{0}^{1} \mathrm{~d} \zeta \frac{1}{(2 \pi)^{3 / 2}} h^{j}\left(\zeta,\left|\mathbf{p}_{T}\right|\right) \cdot \\
& \frac{1}{2}\left(\lambda_{a_{j}}\right)_{A_{j} A_{j}^{\prime}}\left(\boldsymbol{\epsilon}_{j} \cdot \boldsymbol{\sigma} \epsilon\right)_{s_{j} s_{j}^{\prime}}\left|q\left(p_{j}, s_{j}, A_{j}\right) \bar{q}\left(P_{j}-p_{j}, s_{j}^{\prime}, A_{j}^{\prime}\right)\right\rangle \\
& (j=1,2,3,4),
\end{aligned}
$$

where $p_{j}$ is as in (3.67), (3.68) and

$$
\epsilon=\left(\begin{array}{cc}
0 & 1 \\
-1 & 0
\end{array}\right) \text {. }
$$


We require the functions $h^{j}$ to satisfy:

$$
\begin{aligned}
& \left(h^{j}\left(\zeta,\left|\mathbf{p}_{T}\right|\right)\right)^{*}=h^{j}\left(\zeta,\left|\mathbf{p}_{T}\right|\right), \\
& h^{j}\left(\zeta,\left|\mathbf{p}_{T}\right|\right)=h^{j}\left(1-\zeta,\left|\mathbf{p}_{T}\right|\right), \\
& \int \mathrm{d}^{2} p_{T} \int_{0}^{1} \mathrm{~d} \zeta 2 \zeta(1-\zeta)\left|h^{j}\left(\zeta,\left|\mathbf{p}_{T}\right|\right)\right|^{2}=1 .
\end{aligned}
$$

This gives us the normalization of the states (B.4) as

$$
\begin{aligned}
& \left\langle q \bar{q} ; P_{j}^{\prime}, \boldsymbol{\epsilon}_{j}^{\prime}, a_{j}^{\prime} \mid q \bar{q}, P_{j}, \boldsymbol{\epsilon}_{j}, a_{j}\right\rangle \\
& =\boldsymbol{\epsilon}_{j}^{\prime *} \cdot \boldsymbol{\epsilon}_{j} \delta_{a_{j^{\prime}}, a_{j}}(2 \pi)^{3} 2 P_{j}^{0} \delta^{3}\left(\mathbf{P}_{j}^{\prime}-\mathbf{P}_{j}\right) .
\end{aligned}
$$

The $q \bar{q}$ states (B.4) have the same transformation properties under a parity $(\mathrm{P})$ charge conjugation $(\mathrm{C})$ and time reversal $(\mathrm{T})$ transformation as a gluon state:

$$
\begin{aligned}
& U(\mathrm{P})\left|q \bar{q} ; P^{0}, \mathbf{P}, \boldsymbol{\epsilon}, a\right\rangle=-\left|q \bar{q} ; P^{0},-\mathbf{P}, \boldsymbol{\epsilon}, a\right\rangle \\
& U(\mathrm{C})\left|q \bar{q} ; P^{0}, \mathbf{P}, \boldsymbol{\epsilon}, a\right\rangle=-\left|q \bar{q} ; P^{0}, \mathbf{P}, \boldsymbol{\epsilon}, b\right\rangle \cdot \frac{1}{2} \operatorname{Tr}\left(\lambda_{b} \lambda_{a}^{T}\right), \\
& V(\mathrm{~T})\left|q \bar{q} ; P^{0}, \mathbf{P}, \boldsymbol{\epsilon}, a\right\rangle=-\left|q \bar{q} ; P^{0},-\mathbf{P}, \boldsymbol{\epsilon}^{*}, b\right\rangle \frac{1}{2} \operatorname{Tr}\left(\lambda_{b} \lambda_{a}^{T}\right) .
\end{aligned}
$$

Here $U(\mathrm{P}), U(\mathrm{C})$ are the unitary operators, $V(\mathrm{~T})$ is the antiunitary operator representing the $\mathrm{P}, \mathrm{C}$ and $\mathrm{T}$ transformations, respectively, in the Fock space of parton states.

As in (3.71) we define the wave functions in transverse position space and longitudinal momentum fraction:

$$
\varphi^{j}\left(\zeta, \mathbf{y}_{T}\right):=\sqrt{2 \zeta(1-\zeta)} \frac{1}{2 \pi} \int \mathrm{d}^{2} p_{T} \exp \left(i \mathbf{p}_{T} \cdot \mathbf{y}_{T}\right) h^{j}\left(\zeta,\left|\mathbf{p}_{T}\right|\right)
$$

Here we also define for the right (left) movers the wave functions in $y_{-}, \mathbf{y}_{T}$ space $\left(y_{+}, \mathbf{y}_{T}\right.$ space $)$ as:

$$
\begin{aligned}
& \tilde{\varphi}^{j}\left(y_{-}, \mathbf{y}_{T}\right):=\frac{1}{\sqrt{4 \pi}} \sqrt{P_{j+}} \int_{0}^{1} \mathrm{~d} \zeta \varphi^{j}\left(\zeta, \mathbf{y}_{T}\right) \exp \left[-\frac{i}{2} P_{j+}\left(\zeta-\frac{1}{2}\right) y_{-}\right] \\
& (j \text { odd }), \\
& \tilde{\varphi}^{j}\left(y_{+}, \mathbf{y}_{T}\right):=\frac{1}{\sqrt{4 \pi}} \sqrt{P_{j-}} \int_{0}^{1} \mathrm{~d} \zeta \varphi^{j}\left(\zeta, \mathbf{y}_{T}\right) \exp \left[-\frac{i}{2} P_{j-}\left(\zeta-\frac{1}{2}\right) y_{+}\right], \\
& (j \text { even }),
\end{aligned}
$$


The normalization condition (B.6) implies:

$$
\begin{array}{ll}
\int \mathrm{d}^{2} y_{T} \int_{0}^{1} \mathrm{~d} \zeta\left|\varphi^{j}\left(\zeta, \mathbf{y}_{T}\right)\right|^{2}=1 \\
\int \mathrm{d} y_{-} \int \mathrm{d}^{2} y_{T}\left|\tilde{\varphi}^{j}\left(y_{-}, \mathbf{y}_{T}\right)\right|^{2}=1 & (j \text { odd }) . \\
\int \mathrm{d} y_{+} \int \mathrm{d}^{2} y_{T}\left|\tilde{\varphi}^{j}\left(y_{+}, \mathbf{y}_{T}\right)\right|^{2}=1 & (j \text { even }) .
\end{array}
$$

To realize the condition that the $q \bar{q}$ pair acts like a gluon, we require for right (left) movers that they have similar longitudinal momenta and that their wave function in the relative $q-\bar{q}$ coordinates, $y_{-}, \mathbf{y}_{T}\left(y_{+}, \mathbf{y}_{T}\right)$ is nearly a $\delta$ function. To be concrete, we require:

$$
\varphi^{j}\left(\zeta, \mathbf{y}_{T}\right) \neq 0 \quad \text { only for }\left|\zeta-\frac{1}{2}\right| \leq \xi_{0}
$$

where

$$
0<\xi_{0} \ll \frac{1}{2}
$$

and for $j$ odd:

$$
\tilde{\varphi}^{j}\left(y_{-}, \mathbf{y}_{T}\right) \neq 0 \quad \text { only if } \quad\left|y_{-}\right| \lesssim \frac{1}{P_{+} \xi_{0}} \quad \text { and } \quad\left|\mathbf{y}_{T}\right| \ll a .
$$

For right movers we replace plus by minus signs in (B.17). Any $q \bar{q}$ wave packets with these properties should then look identical to a gluon for an observer in the femto universe with regard to "soft" scatterings.

Now we replace $G(1)$ and $G(3)$ in reaction $(\mathbb{B} .1)$ by the $q \bar{q}$ wave packets (B.4). According to the rules derived in sect. 3.5 the scattering of the $q \bar{q}$ system

$$
q(1) \bar{q}\left(1^{\prime}\right) \rightarrow q(3) \bar{q}\left(3^{\prime}\right)
$$

gives the following factor in the $S$-matrix (cf. (3.58) (3.59)):

$$
\mathcal{S}_{q+}(3,1) \mathcal{S}_{\bar{q}+}\left(3^{\prime}, 1^{\prime}\right)
$$

which still has to be integrated over the wave functions (B.4). In this way we obtain for the $q \bar{q}$-pair: 


$$
\begin{aligned}
& \mathcal{S}_{q \bar{q}}(3,1)= \int \mathrm{d}^{2} p_{T}^{\prime} \int_{0}^{1} \mathrm{~d} \zeta^{\prime} \int \mathrm{d}^{2} p_{T} \int_{0}^{1} \mathrm{~d} \zeta \\
& \frac{1}{(2 \pi)^{3}} h^{3}\left(\zeta^{\prime},\left|\mathbf{p}_{T}^{\prime}\right|\right) h^{1}\left(\zeta,\left|\mathbf{p}_{T}\right|\right) \\
& \frac{1}{2}\left(\lambda_{a_{3}}\right)_{A_{3}^{\prime}, A_{3}}\left(\boldsymbol{\epsilon}_{3}{ }^{*} \cdot \epsilon^{T} \boldsymbol{\sigma}\right)_{s_{3}^{\prime}, s_{3}} \\
& \frac{1}{2}\left(\lambda_{a_{1}}\right)_{A_{1}, A_{1}^{\prime}}\left(\boldsymbol{\epsilon}_{1} \cdot \boldsymbol{\sigma} \epsilon\right)_{s_{1}, s_{1}^{\prime}} \\
& \mathcal{S}_{q+}(3,1) \mathcal{S}_{\bar{q}+}\left(3^{\prime}, 1^{\prime}\right), \\
& \mathcal{S}_{q \bar{q}}(3,1)= \int \mathrm{d} z_{-} \int \mathrm{d} y_{-} \int \mathrm{d}^{2} z_{T} \int \mathrm{d}^{2} y_{T} \sqrt{P_{3+} P_{1+}} \boldsymbol{\epsilon}_{3}^{*} \cdot \boldsymbol{\epsilon}_{1} \\
& \exp \left[\frac{i}{2}\left(P_{3+}-P_{1+}\right) z_{-}-i\left(\mathbf{P}_{3}-\mathbf{P}_{1}\right)_{T} \mathbf{z}_{T}\right] \\
& \tilde{\varphi}^{3 *}\left(y_{-}, \mathbf{y}_{T}\right) \tilde{\varphi}^{1}\left(y_{-}, \mathbf{y}_{T}\right) \\
& \frac{1}{2} \operatorname{Tr}\left[\lambda_{a_{3}} V_{-}\left(\infty, z_{-}+\frac{1}{2} y_{-}, \mathbf{z}_{T}+\frac{1}{2} \mathbf{y}_{T}\right)\right. \\
&\left.\lambda_{a_{1}} V_{-}^{\dagger}\left(\infty, z_{-}-\frac{1}{2} y_{-}, \mathbf{z}_{T}-\frac{1}{2} \mathbf{y}_{T}\right)\right]
\end{aligned}
$$

With our assumptions (B.17) $\tilde{\varphi}^{3 *}\left(y_{-}, \mathbf{y}_{T}\right) \tilde{\varphi}^{1}\left(y_{-}, \mathbf{y}_{T}\right)$ acts like a $\delta$-function at $y_{-}=0, \mathbf{y}_{T}=0$ and we get:

$$
\begin{aligned}
\mathcal{S}_{q \bar{q}}(3,1)= & \sqrt{P_{3+} P_{1+}} \boldsymbol{\epsilon}_{3}^{*} \cdot \boldsymbol{\epsilon}_{1} \\
& \int \mathrm{d} y_{-} \int \mathrm{d}^{2} y_{T} \tilde{\varphi}^{3 *}\left(y_{-}, \mathbf{y}_{T}\right) \tilde{\varphi}^{1}\left(y_{-}, \mathbf{y}_{T}\right) \\
& \int \mathrm{d} z_{-} \int \mathrm{d}^{2} z_{T} \exp \left[\frac{i}{2}\left(P_{3+}-P_{1+}\right) z_{-}-i\left(\mathbf{P}_{3}-\mathbf{P}_{1}\right)_{T} \cdot \mathbf{z}_{T}\right] \\
& \frac{1}{2} \operatorname{Tr}\left[\lambda_{a_{3}} V_{-}\left(\infty, z_{-}, \mathbf{z}_{T}\right) \lambda_{a_{1}} V_{-}^{\dagger}\left(\infty, z_{-}, \mathbf{z}_{T}\right)\right]
\end{aligned}
$$

An easy exercise shows that

$$
\frac{1}{2} \operatorname{Tr}\left[\lambda_{a_{3}} V_{-}\left(\infty, z_{-}, \mathbf{z}_{T}\right) \lambda_{a_{1}} V_{-}^{\dagger}\left(\infty, z_{-}, \mathbf{z}_{T}\right)\right]=\mathcal{V}_{-}\left(\infty, z_{-}, \mathbf{z}_{T}\right)_{a_{3}, a_{1}}
$$


where $\mathcal{V}_{-}$is the connector analogous to (3.35) but for the adjoint representation (cf. (2.22)):

$$
\begin{aligned}
& \mathcal{V}_{-}\left(\infty, z_{-}, \mathbf{z}_{T}\right)=\mathrm{P}\left\{\exp \left[-\frac{i}{2} g \int_{-\infty}^{\infty} \mathrm{d} z_{+} G_{-}^{a}\left(z_{+}, z_{-}, \mathbf{z}_{T}\right) T_{a}\right]\right\}, \\
& \left(T_{a}\right)_{b c}=\frac{1}{i} f_{a b c} .
\end{aligned}
$$

The $f_{a b c}$ are the structure constants of $S U(3)_{c}$. Inserting (B.22) in (B.21) gives

$$
\mathcal{S}_{q \bar{q}}(3,1)=\int \mathrm{d} y_{-} \int \mathrm{d}^{2} y_{T} \tilde{\varphi}^{3 *}\left(y_{-}, \mathbf{y}_{T}\right) \tilde{\varphi}^{1}\left(y_{-}, \mathbf{y}_{T}\right) \epsilon_{3 j_{3}}^{*} \epsilon_{1 j_{1}} \mathcal{S}_{G+}(3,1),
$$

where

$$
\begin{aligned}
& \mathcal{S}_{G+}(3,1)=\sqrt{P_{3+} P_{1+}} \delta_{j_{3}, j_{1}} \int \mathrm{d} z_{-} \int \mathrm{d}^{2} z_{T} \\
& \exp \left[\frac{i}{2}\left(P_{3+}-P_{1+}\right) z_{-}-i\left(\mathbf{P}_{3 T}-\mathbf{P}_{1 T}\right) \cdot \mathbf{z}_{T}\right] \\
& \mathcal{V}_{-}\left(\infty, z_{-}, \mathbf{z}_{T}\right)_{a_{3}, a_{1}} .
\end{aligned}
$$

In a general scattering reaction $(\mathbb{B . 1})$ the factor $\mathcal{S}_{q \bar{q}}(3,1)$ (B.24) has to be inserted with other factors $\mathcal{S}_{q}, \mathcal{S}_{\bar{q}}, \ldots$ and then integrated over all gluon potentials as explained in sect. 3.5. We note that $\mathcal{S}_{q \bar{q}}(3,1)$ in (B.24) factorizes into $\mathcal{S}_{G+}(3,1)($ B.25) times the overlap of the internal wave functions of the incoming and outgoing $q \bar{q}$ pairs:

$$
\int \mathrm{d} y_{-} \int \mathrm{d}^{2} y_{T} \tilde{\varphi}^{3 *}\left(y_{-}, \mathbf{y}_{T}\right) \tilde{\varphi}^{1}\left(y_{-}, \mathbf{y}_{T}\right) .
$$

This means that the $q \bar{q}$ pair will come out with some distribution in total momentum $P_{3}$ and polarization vector $\boldsymbol{\epsilon}_{3}=\boldsymbol{\epsilon}_{1}$ but always with internal wave function $\tilde{\varphi}^{1}\left(y_{-}, \mathbf{y}_{T}\right)$. With the conditions B.15)-(B.17) $\tilde{\varphi}^{1}\left(y_{-}, \mathbf{y}_{T}\right)$ leads to a "permissible" internal wave function for a $q \bar{q}$ pair of momentum $P_{3}$, to be regarded as a gluon of momentum $P_{3}$ by our observer in the femto universe. Indeed we have from $(\mathbb{B . 1 2})$ :

$$
\begin{aligned}
\tilde{\varphi}^{1}\left(y_{-}, \mathbf{y}_{T}\right) & =\sqrt{\frac{P_{1+}}{4 \pi}} \int_{0}^{1} \mathrm{~d} \zeta \varphi^{1}\left(\zeta, \mathbf{y}_{T}\right) \exp \left[-\frac{i}{2} P_{1+}\left(\zeta-\frac{1}{2}\right) y_{-}\right] \\
& =\sqrt{\frac{P_{1+}}{4 \pi}} \int_{-1 / 2}^{1 / 2} \mathrm{~d} \xi \varphi^{1}\left(\frac{1}{2}+\xi, \mathbf{y}_{T}\right) \exp \left[-\frac{i}{2} P_{1+} \xi \cdot y_{-}\right] \\
& =\sqrt{\frac{P_{3+}}{4 \pi}} \int_{\xi-}^{\xi+} \mathrm{d} \xi^{\prime} \varphi^{\prime 3}\left(\frac{1}{2}+\xi^{\prime}, \mathbf{y}_{T}\right) \exp \left[-\frac{i}{2} P_{3+} \xi^{\prime} \cdot y_{-}\right]
\end{aligned}
$$


Here we define the internal wave function of the outgoing $q \bar{q}$ pair as

$$
\varphi^{\prime 3}\left(\frac{1}{2}+\xi^{\prime}, \mathbf{y}_{T}\right)=\sqrt{\frac{P_{3+}}{P_{1+}}} \varphi^{1}\left(\frac{1}{2}+\frac{P_{3+}}{P_{1+}} \xi^{\prime}, \mathbf{y}_{T}\right)
$$

and

$$
\xi_{ \pm}= \pm \frac{P_{1+}}{P_{3+}} \cdot \frac{1}{2}
$$

The condition (B.15) for $\varphi^{1}$ guarantees a similar condition for $\varphi^{\prime 3}$ if $P_{3+} / P_{1+}$ is of order 1 , as we will always assume. Thus the integration limits $\xi_{ \pm}$in

(B.27) can be replaced by $\pm \frac{1}{2}$ and also the normalization conditions (B.14) can easily be checked for $\varphi^{\prime 3}$.

To summarize: In this appendix we have shown that suitable $q \bar{q}$ pairs which are indistinguishable from gluons for our observer in the femto universe scatter as entities in a soft reaction. Their internal wave function in momentum space is modified, but in a way not observable in a soft reaction. Thus we can consider $\mathcal{S}_{G+}(3,1)$ in $(\mathrm{B} .25)$ as the scattering contribution of a right-moving gluon in the transition (B.2). We quoted this result already in (3.64) in sect. 3.5. For left moving gluons we just have to exchange everywhere + and - components.

\section{Appendix C: The scattering of baryons}

In this appendix we discuss high energy soft reactions, in particular elastic reactions involving baryons and antibaryons. We represent baryons by $q q q$ wave packets:

$$
\begin{aligned}
& \left|B_{j}\left(P_{j}\right)\right\rangle=\frac{1}{6 \cdot(2 \pi)^{3}} \int \mathrm{d} \mu h^{j}\left(f^{i}, s^{i}, \zeta^{i}, \mathbf{p}_{T}^{i}\right) \cdot \\
& \epsilon_{A^{1} A^{2} A^{3}}\left|q\left(p_{j}^{1}, f^{1}, s^{1}, A^{1}\right), q\left(p_{j}^{2}, f^{2}, s^{2}, A^{2}\right), q\left(p_{j}^{3}, f^{3}, s^{3}, A^{3}\right)\right\rangle .
\end{aligned}
$$

Here $f^{i}, s^{i}, A^{i}(i=1,2,3)$ are the flavour, spin, and colour indices of the quarks and $\epsilon_{A B C}\left(\epsilon_{123}=1\right)$ is the totally antisymmetric tensor. For the momenta $p_{j}^{i}$ we set for right-moving baryons ( $j$ odd, $P_{j+} \rightarrow \infty$ ):

$$
\begin{aligned}
& p_{j+}^{i}=\zeta^{i} P_{j+}, \\
& \mathbf{p}_{j T}^{i}=\frac{1}{3} \mathbf{P}_{j T}+\mathbf{p}_{T}^{i}, \\
& (i=1,2,3)
\end{aligned}
$$


and for left-moving baryons $\left(j\right.$ even, $\left.P_{j-} \rightarrow \infty\right)$ :

$$
\begin{aligned}
& p_{j-}^{i}=\zeta^{i} P_{j-}, \\
& \mathbf{p}_{j T}^{i}=\frac{1}{3} \mathbf{P}_{j T}+\mathbf{p}_{T}^{i}, \\
& (i=1,2,3) .
\end{aligned}
$$

The integral with the measure $\mathrm{d} \mu$ stands for

$$
\begin{aligned}
\int \mathrm{d} \mu \equiv & \iiint \prod_{i=1}^{3} \mathrm{~d}^{2} p_{T}^{i} \delta^{2}\left(\sum_{i=1}^{3} \mathbf{p}_{T}^{i}\right) . \\
& \int_{0}^{1} \int_{0}^{1} \int_{0}^{1} \prod_{i=1}^{3} \mathrm{~d} \zeta^{i} \delta\left(1-\sum_{i=1}^{3} \zeta^{i}\right) .
\end{aligned}
$$

The flavour and spin of the baryon states (C.1) is, of course, determined by the functions $h^{j}$ which must be totally symmetric under simultaneous exchange of the arguments $\left(f^{i}, s^{i}, \zeta^{i}, \mathbf{p}_{T}^{i}\right)$ for $i=1,2,3$. In the following we will collectively set $\left(f, s, \zeta, \mathbf{p}_{T}\right) \equiv \alpha$ and $q(\alpha, A) \equiv q\left(p_{j}, f, s, A\right)$. We have then

$$
\begin{aligned}
& h^{j}(\alpha, \beta, \gamma)=h^{j}(\beta, \alpha \gamma)=h^{j}(\alpha, \gamma, \beta) . \\
& \left|B_{j}\left(P_{j}\right)\right\rangle=\frac{1}{6 .(2 \pi)^{3}} \int \mathrm{d} \mu h^{j}(\alpha, \beta, \gamma) \epsilon_{A B C}|q(\alpha, A) q(\beta, B) q(\gamma, C)\rangle .
\end{aligned}
$$

The normalization condition

$$
\left\langle B_{j}\left(P_{j}^{\prime}\right) \mid B_{j}\left(P_{j}\right)\right\rangle=(2 \pi)^{3} 2 P_{j}^{0} \delta^{3}\left(\mathbf{P}_{j}^{\prime}-\mathbf{P}_{j}\right)
$$

requires $h^{j}$ to satisfy:

$$
\begin{aligned}
& \int \mathrm{d} \mu 4 \zeta^{1} \zeta^{2} \zeta^{3} h^{j^{*}}(\alpha, \beta, \gamma) h^{j}(\alpha, \beta, \gamma)=1 \\
& \text { (no summation over } j) .
\end{aligned}
$$

We define the wave functions $\varphi^{j}$ in transverse position and longitudinal momentum space and the transition profile functions $w_{k j}^{B}$ for $B_{j} \rightarrow B_{k}$ as: 


$$
\begin{aligned}
& \varphi^{j}\left(f^{i}, s^{i}, \zeta^{i}, \mathbf{x}_{T}^{i}\right):=\frac{1}{(2 \pi)^{2}} \int \prod_{i=1}^{3} \mathrm{~d}^{2} p_{T}^{i} \cdot \delta^{2}\left(\sum_{i=1}^{3} \mathbf{p}_{T}^{i}\right) \cdot \\
& 6 \cdot\left(\zeta^{1} \zeta^{2} \zeta^{3}\right)^{1 / 2} \exp \left(i \sum_{i=1}^{3} \mathbf{p}_{T}^{i} \cdot \mathbf{x}_{T}^{i}\right) h^{j}\left(\alpha^{1}, \alpha^{2}, \alpha^{3}\right), \\
& w_{k, j}^{B}\left(\mathbf{x}_{T}^{1}, \mathbf{x}_{T}^{2}, \mathbf{x}_{T}^{3}\right):=\int_{0}^{1} \int_{0}^{1} \int_{0}^{1} \prod_{i=1}^{3} \mathrm{~d} \zeta^{i} \delta\left(1-\sum_{i=1}^{3} \zeta^{i}\right) \\
& \sum_{f^{i}, s^{i}} \varphi^{3^{*}}\left(f^{1}, s^{1}, \zeta^{1}, \mathbf{x}_{T}^{1} ; f^{2}, s^{2}, \zeta^{2}, \mathbf{x}_{T}^{2} ; f^{3}, s^{3}, \zeta^{3}, \mathbf{x}_{T}^{3}\right) \\
& \varphi^{1}\left(f^{1}, s^{1}, \zeta^{1}, \mathbf{x}_{T}^{1} ; f^{2}, s^{2}, \zeta^{2}, \mathbf{x}_{T}^{2} ; f^{3}, s^{3}, \zeta^{3}, \mathbf{x}_{T}^{3}\right) .
\end{aligned}
$$

The symmetry relations for $h($ C.5 $)$ and the normalization condition (C.8) imply:

$$
\begin{gathered}
w_{k, j}^{B}\left(\mathbf{x}_{T}^{1}, \mathbf{x}_{T}^{2}, \mathbf{x}_{T}^{3}\right)=w_{k, j}^{B}\left(\mathbf{x}_{T}^{2}, \mathbf{x}_{T}^{1}, \mathbf{x}_{T}^{3}\right)=w_{k, j}^{B}\left(\mathbf{x}_{T}^{1}, \mathbf{x}_{T}^{3}, \mathbf{x}_{T}^{2}\right), \\
\int \prod_{i=1}^{3} \mathrm{~d}^{2} x_{T}^{i} \delta^{2}\left(\mathbf{x}_{T}^{1}+\mathbf{x}_{T}^{2}+\mathbf{x}_{T}^{3}\right) w_{j j}^{B}\left(\mathbf{x}_{T}^{1}, \mathbf{x}_{T}^{2}, \mathbf{x}_{T}^{3}\right)=1 \\
(\text { no summation over } j) .
\end{gathered}
$$

As a concrete scattering reaction let us consider meson-baryon scattering:

$$
B_{1}\left(P_{1}\right)+M_{2}\left(P_{2}\right) \rightarrow B_{3}\left(P_{3}\right)+M_{4}\left(P_{4}\right)
$$

From the rules of sect. 3.5 we get

$$
\begin{aligned}
& S_{f i} \equiv\left\langle B_{3}\left(P_{3}\right), M_{4}\left(P_{4}\right)|S| B_{1}\left(P_{1}\right) M_{2}\left(P_{2}\right)\right\rangle= \\
& \frac{1}{6(2 \pi)^{6}} \int \mathrm{d} \mu^{\prime} h^{3^{*}}\left(\alpha^{\prime}, \beta^{\prime}, \gamma^{\prime}\right) \epsilon_{A^{\prime} B^{\prime} C^{\prime}} \int \mathrm{d} \mu h^{1}(\alpha, \beta, \gamma) \epsilon_{A B C} \\
& \frac{1}{3(2 \pi)^{3}} \int \mathrm{d}^{2} p_{T}^{\prime} \int_{0}^{1} \mathrm{~d} \zeta^{\prime} \int \mathrm{d}^{2} p_{T} \int_{0}^{1} \mathrm{~d} \zeta h^{4^{*}{ }_{s_{4} s_{4}^{\prime}}}\left(\zeta^{\prime}, \mathbf{p}_{T}^{\prime}\right) h_{s_{2} s_{2}^{\prime}}^{2}\left(\zeta, \mathbf{p}_{T}\right) \\
& \left\langle\mathcal{S}_{q+}\left(\alpha^{\prime}, \alpha\right)_{A^{\prime} A_{\mathcal{A}}} \mathcal{S}_{q+}\left(\beta^{\prime}, \beta\right)_{B^{\prime} B} \mathcal{S}_{q+}\left(\gamma^{\prime}, \gamma\right)_{C^{\prime} C}\right. \\
& \left.\delta_{A_{2}^{\prime} A_{2}} \delta_{A_{4}^{\prime} A_{4}} \mathcal{S}_{q-}(4,2)_{A_{4} A_{2}} \mathcal{S}_{\bar{q}-}\left(4^{\prime}, 2^{\prime}\right)_{A_{4}^{\prime} A_{2}^{\prime}}\right\rangle_{G} .
\end{aligned}
$$


After some straightforward algebra we get:

$$
\begin{aligned}
S_{f i}= & \delta_{f i}+i(2 \pi)^{4} \delta\left(P_{3}+P_{4}-P_{1}-P_{2}\right) \mathcal{T}_{f i}, \\
\mathcal{T}_{f i}= & -\frac{i}{(2 \pi)^{6}} \int \mathrm{d} \mu^{\prime} h^{3^{*}}\left(\alpha^{\prime}, \beta^{\prime}, \gamma^{\prime}\right) \\
& \int \mathrm{d} \mu h^{1}(\alpha, \beta, \gamma) \prod_{i=1}^{3}\left(\delta_{f^{\prime i}, f^{i}} \delta_{s^{\prime}, s^{i}}\right)\left(P_{1+}\right)^{3}\left[\prod_{i=1}^{3} \zeta^{\prime i} \zeta^{i}\right]^{1 / 2} \\
& \frac{1}{(2 \pi)^{3}} \int \mathrm{d}^{2} p_{T}^{\prime} \int_{0}^{1} \mathrm{~d} \zeta^{\prime} \int \mathrm{d}^{2} p_{T} \int_{0}^{1} \mathrm{~d} \zeta h^{4^{*}, r}\left(\zeta^{\prime}, \mathbf{p}_{T}^{\prime}\right) h_{s, r}^{2}\left(\zeta, \mathbf{p}_{T}\right) \\
& 2\left(P_{2-}\right)^{2}\left[\zeta^{\prime}\left(1-\zeta^{\prime}\right) \zeta(1-\zeta)\right]^{1 / 2} \\
& 3^{3} \cdot \int \mathrm{d}^{2} b_{T} \exp \left(i \mathbf{q}_{T} \cdot \mathbf{b}_{T}\right) \int \prod_{i=1}^{3}\left(\mathrm{~d} x_{-}^{i} \mathrm{~d}^{2} x_{T}^{i}\right) \delta\left(\sum_{i=1}^{3} x_{-}^{i}\right) \delta^{2}\left(\sum_{i=1}^{3} \mathbf{x}_{T}^{i}\right) \\
& \int \mathrm{d} y_{+} \mathrm{d}^{2} y_{T} \exp \left\{i \sum_{i=1}^{3}\left[\frac{1}{2} P_{1+}\left(\zeta^{\prime i}-\zeta^{i}\right) x_{-}^{i}-\left(\mathbf{p}_{T}^{\prime i}-\mathbf{p}_{T}^{i}\right) \cdot \mathbf{x}_{T}^{i}\right]\right. \\
& \left.+i \frac{1}{2} P_{2-}\left(\zeta^{\prime}-\zeta\right) y_{+}-i\left(\mathbf{p}_{T}^{\prime}-\mathbf{p}_{T}\right) \cdot \mathbf{y}_{T}\right\} \\
& \left\langle\left\{ V_{-}\left(\infty, x_{-}^{1}, \frac{1}{2} \mathbf{b}_{T}+\mathbf{x}_{T}^{1}\right)_{A^{\prime} A} V_{-}\left(\infty, x_{-}^{2}, \frac{1}{2} \mathbf{b}_{T}+\mathbf{x}_{T}^{2}\right)_{B^{\prime} B}\right.\right. \\
& V_{-}\left(\infty, x_{-}^{3}, \frac{1}{2} \mathbf{b}_{T}+\mathbf{x}_{T}^{3}\right)_{C^{\prime} C} \frac{1}{6} \varepsilon_{A^{\prime} B^{\prime} C^{\prime}} \varepsilon_{A B C} \\
& \left.\left.\frac{1}{3} \operatorname{Tr}_{A}\left[V_{+}\left(y_{+}, \infty,-\frac{1}{2} \mathbf{b}_{T}+\frac{1}{2} \mathbf{y}_{T}\right) V_{+}^{\dagger}\left(0, \infty,-\frac{1}{2} \mathbf{b}_{T}-\frac{1}{2} \mathbf{y}_{T}\right)\right]-1\right\}\right\rangle_{G},
\end{aligned}
$$

where

$$
\mathbf{q}_{T}=\left(\mathbf{P}_{1}-\mathbf{P}_{3}\right)_{T}
$$

Now we make the transformation of variables

$$
\begin{aligned}
& x_{-}^{i} \rightarrow \frac{2}{P_{1+}} x_{-}^{i}, \\
& y_{+} \rightarrow \frac{2}{P_{2-}} y_{+}
\end{aligned}
$$

and use $P_{1+} \rightarrow \infty, P_{2-} \rightarrow \infty$. With the same arguments which led us from 
(3.77) to (3.79) we get

$$
\begin{aligned}
\mathcal{T}_{f i}= & -2 i s \int \mathrm{d}^{2} b_{T} \exp \left(i \mathbf{q}_{T} \cdot \mathbf{b}_{T}\right) \int \prod_{i=1}^{3} \mathrm{~d}^{2} x_{T}^{i} \delta^{2}\left(\mathbf{x}_{T}^{1}+\mathbf{x}_{T}^{2}+\mathbf{x}_{T}^{3}\right) \\
& w_{3,1}^{B}\left(\mathbf{x}_{T}^{1}, \mathbf{x}_{T}^{2}, \mathbf{x}_{T}^{3}\right) \int \mathrm{d}^{2} y_{T} w_{4,2}^{M}\left(\mathbf{y}_{T}\right) \\
\langle\{ & V_{-}\left(\infty, 0, \frac{1}{2} \mathbf{b}_{T}+\mathbf{x}_{T}^{1}\right)_{A^{\prime} A} V_{-}\left(\infty, 0, \frac{1}{2} \mathbf{b}_{T}+\mathbf{x}_{T}^{2}\right)_{B^{\prime} B} \\
& V_{-}\left(\infty, 0, \frac{1}{2} \mathbf{b}_{T}+\mathbf{x}_{T}^{3}\right)_{C^{\prime} C} \frac{1}{6} \epsilon_{A^{\prime} B^{\prime} C^{\prime}} \epsilon_{A B C} \\
& \left.\frac{1}{3} \operatorname{Tr}\left[V_{+}\left(0, \infty,-\frac{1}{2} \mathbf{b}_{T}+\frac{1}{2} \mathbf{y}_{T}\right) V_{+}^{\dagger}\left(0, \infty,-\frac{1}{2} \mathbf{b}_{T}-\frac{1}{2} \mathbf{y}_{T}\right]-1\right\}\right\rangle_{G} .
\end{aligned}
$$

Here $w_{4,2}^{M}\left(y_{T}\right)$ is the transition profile function for the mesons as defined in (3.72) and $w_{3,1}^{B}$ is the corresponding function for the baryons (cf. (C.10)).

In the next step we follow [59], 45] and use relations which are valid for any $3 \times 3$ matrix: $H=\left(H_{A B}\right)$ :

$$
\begin{aligned}
& \quad H_{A^{\prime} A^{\prime \prime}} H_{B^{\prime} B^{\prime \prime}} H_{C^{\prime} C^{\prime \prime}} \cdot \epsilon_{A^{\prime \prime} B^{\prime \prime} C^{\prime \prime}}=\operatorname{det} H \cdot \epsilon_{A^{\prime} B^{\prime} C^{\prime}}, \\
& \operatorname{det} H \cdot \epsilon_{A^{\prime} B^{\prime} C^{\prime}} \epsilon_{A B C}= \\
& H_{A^{\prime} A} H_{B^{\prime} B} H_{C^{\prime} C}+H_{A^{\prime} B} H_{B^{\prime} C} H_{C^{\prime} A}+H_{A^{\prime} C} H_{B^{\prime} A} H_{C^{\prime} B} \\
& -H_{A^{\prime} B} H_{B^{\prime} A} H_{C^{\prime} C}-H_{A^{\prime} A} H_{B^{\prime} C} H_{C^{\prime} B}-H_{A^{\prime} C} H_{B^{\prime} B} H_{C^{\prime} A} .
\end{aligned}
$$

We take $H$ equal to the antiquark line integral at the central point of the baryon in transverse space:

$$
H=V_{-}^{*}\left(\infty, 0, \frac{1}{2} \mathbf{b}_{T}\right)
$$

As a $S U(3)$-connector $V_{-}^{*}$ satisfies

$$
\operatorname{det} V_{-}^{*}\left(\infty, 0, \frac{1}{2} \mathbf{b}_{T}\right)=1
$$

This is easy to prove. From (C.19) we see that $\operatorname{det} V_{-}^{*}$ is the connector in the singlet part of the product of three $S U(3)$ antiquark representations: 
$\overline{3} \times \overline{3} \times \overline{3}$. But for the singlet representation the connector equals 1 since we have to set $T_{a}=0$ in (2.22).

In the following we will use as shorthand notation

$$
\begin{aligned}
& V(i) \equiv V_{-}\left(\infty, 0, \frac{1}{2} \mathbf{b}_{T}+\mathbf{x}_{T}^{i}\right), \\
& (i=1,2,3), \\
& V(0) \equiv V_{-}\left(\infty, 0, \frac{1}{2} \mathbf{b}_{T}\right) .
\end{aligned}
$$

With this we get for the $q q q$-contribution to the integrand in the functional integral in (C.18) using (C.20) to (C.22):

$$
\begin{aligned}
\mathcal{W}_{+}^{B}( & \left.\frac{1}{2} \mathbf{b}_{T}, \mathbf{x}_{T}^{1}, \mathbf{x}_{T}^{2}, \mathbf{x}_{T}^{3}\right):=\frac{1}{6} V(1)_{A^{\prime} A} V(2)_{B^{\prime} B} V(3)_{C^{\prime} C} \epsilon_{A^{\prime} B^{\prime} C^{\prime}} \epsilon_{A B C} \\
=\frac{1}{6}\{ & \operatorname{Tr}\left[V(1) V^{\dagger}(0)\right] \cdot \operatorname{Tr}\left[V(2) V^{\dagger}(0)\right] \cdot \operatorname{Tr}\left[V(3) V^{\dagger}(0)\right] \\
& +\operatorname{Tr}\left[V(1) V^{\dagger}(0) V(2) V^{\dagger}(0) V(3) V^{\dagger}(0)\right] \\
& +\operatorname{Tr}\left[V(1) V^{\dagger}(0) V(3) V^{\dagger}(0) V(2) V^{\dagger}(0)\right] \\
& \quad-\operatorname{Tr}\left[V(1) V^{\dagger}(0) V(2) V^{\dagger}(0)\right] \cdot \operatorname{Tr}\left[V(3) V^{\dagger}(0)\right] \\
& -\operatorname{Tr}\left[V(2) V^{\dagger}(0) V(3) V^{\dagger}(0)\right] \cdot \operatorname{Tr}\left[V(1) V^{\dagger}(0)\right] \\
& \left.-\operatorname{Tr}\left[V(1) V^{\dagger}(0) V(3) V^{\dagger}(0)\right] \cdot \operatorname{Tr}\left[V(2) V^{\dagger}(0)\right]\right\}
\end{aligned}
$$

As for the meson case (cf. (3.82)) we will now add suitable connectors at $\pm \infty$. In this way all the traces in (C.24) become closed light-like Wegner-Wilson loops. To give an example: the term

$$
\operatorname{Tr}\left[V(1) V^{\dagger}(0) V(2) V^{\dagger}(0)\right]
$$

should then be read as the loop in the hyperplane $x_{-}=0$ in the limit $T \rightarrow \infty$ which connects the following points $\left(x_{+}, x_{-}, \mathbf{x}_{T}\right)$ :

$$
\begin{aligned}
& \left(T, 0, \frac{1}{2} \mathbf{b}_{T}\right), \\
& \left(-T, 0, \frac{1}{2} \mathbf{b}_{T}\right), \\
& \left(-T, 0, \frac{1}{2} \mathbf{b}_{T}+\mathbf{x}_{T}^{2}\right),
\end{aligned}
$$




$$
\begin{aligned}
& \left(T, 0, \frac{1}{2} \mathbf{b}_{T}+\mathbf{x}_{T}^{2}\right), \\
& \left(T, 0, \frac{1}{2} \mathbf{b}_{T}\right), \\
& \left(-T, 0, \frac{1}{2} \mathbf{b}_{T}\right), \\
& \left(-T, 0, \frac{1}{2} \mathbf{b}_{T}+\mathbf{x}_{T}^{1}\right), \\
& \left(T, 0, \frac{1}{2} \mathbf{b}_{T}+\mathbf{x}_{T}^{1}\right), \\
& \left(T, 0, \frac{1}{2} \mathbf{b}_{T}\right)
\end{aligned}
$$

on straight lines in the order indicated.

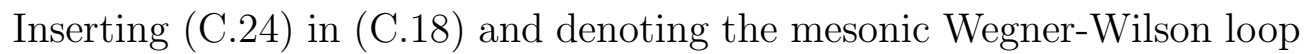
as defined in (3.83) by $\mathcal{W}_{-}^{M}$, we get finally for the $T$-matrix element of baryonmeson scattering:

$$
\begin{aligned}
& \mathcal{T}_{f i}=-2 i s \int \mathrm{d}^{2} b_{T} \exp \left(i \mathbf{q}_{T} \cdot \mathbf{b}_{T}\right) \\
& \int \prod_{i=1}^{3} \mathrm{~d}^{2} x_{T}^{i} \delta^{2}\left(\mathbf{x}_{T}^{1}+\mathbf{x}_{T}^{2}+\mathbf{x}_{T}^{3}\right) w_{3,1}^{B}\left(\mathbf{x}_{T}^{1}, \mathbf{x}_{T}^{2}, \mathbf{x}_{T}^{3}\right) \\
& \int \mathrm{d}^{2} y_{T} w_{4,2}^{M}\left(\mathbf{y}_{T}\right) \\
& \left\langle\mathcal{W}_{+}^{B}\left(\frac{1}{2} \mathbf{b}_{T}, \mathbf{x}_{T}^{1}, \mathbf{x}_{T}^{2}, \mathbf{x}_{T}^{3}\right) \mathcal{W}_{-}^{M}\left(-\frac{1}{2} \mathbf{b}_{T}, \mathbf{y}_{T}\right)-1\right\rangle_{G} .
\end{aligned}
$$

This formula is the starting point for the evaluation of the baryon-meson elastic scattering amplitude: One can now apply the Minkowskian version of the SVM to calculate the functional integral \langle\rangle$_{G}$ in an appropriate way. Then one has to fold the result with the profile functions of the mesonic and baryonic transitions. At the present state one has to make a suitable ansatz for these profile functions.

For the case of right-moving antibaryons in an elastic reaction we just have to substitute the loop factor $\mathcal{W}_{+}^{B}$ by $\mathcal{W}_{+}^{\bar{B}}$ which is obtained by replacing the quark connectors $V_{-}$by the antiquark connectors $V_{-}^{*}$ and vice versa in (C.23), (C.24). In an equivalent way we can get $W_{+}^{\bar{B}}$ from $\mathcal{W}_{+}^{B}$ by reversing the directional arrows on all Wegner-Wilson loops obtained in the way discussed 
above from (C.24). For left-moving baryons and antibaryons we have to exchange + and - components.

For the further treatment of scattering amplitudes involving mesons, baryons and antibaryons, for many results and a comparison with experiments we refer to [59], 45], [62].

\section{References}

[1] H. Fritzsch, , M. Gell-Mann, H. Leutwyler: Phys. Lett. B47, 365 (1973); M. Gell-Mann: Acta Physica Austriaca, Suppl. 9, 733 (1972)

[2] G. 't Hooft: remarks at a conference in Marseille (1972);

H. D. Politzer: Phys. Rev. Lett. 30, 1346 (1973);

D. Gross, F. Wilczek: Phys. Rev. Lett. 30, 1343 (1973)

[3] K. G. Wilson: Phys. Rev. D10, 2445 (1974)

[4] P. Lepage: "Designer Field Theory-Redesigning Lattice QCD" in "Perturbative and Nonperturbative Aspects of Quantum Field Theory", eds. H. Latal, W. Schweiger, to be published by Springer, Heidelberg

[5] G. Altarelli: Phys. Rep. 81, 1 (1982)

[6] J. L. Cardy, G. A. Winbow: Phys. Lett. B52, 95 (1974);

C. E. DeTar, S. D. Ellis, P. V. Landshoff: Nucl. Phys. B87, 176 (1975)

[7] H. D. Politzer: Nucl. Phys. B129, 301 (1977);

C. T. Sachrajda: Phys. Lett. B73, 185 (1978);

D. Amati, R. Petronzio, G. Veneziano: Nucl. Phys. B140, 54 (1978);

B146, 29 (1978);

R. K. Ellis, H. Georgi, M. Machacek, H. D. Politzer, G. G. Ross: Nucl. Phys. B152, 285 (1979);

S. B. Libby, G. Sterman: Phys. Rev. D18, 3252 (1978);

S. Gupta, A. H. Mueller: Phys. Rev. D20, 118 (1979)

[8] J. C. Collins, D. E. Soper, G. Sterman: Nucl. Phys. B261, 104 (1985); B308, 833 (1988); 
G. Sterman: Phys. Lett. B179, 281 (1986); Nucl. Phys. B281, 310 (1987);

J. C. Collins, D. E. Soper: Ann. Rev. Nucl. Part. Sci. 37, 383 (1987);

T. Matsuura, W. L. van Neerven: Z. Phys. C38, 623 (1988);

J. C. Collins, D. E. Soper, G. Sterman: "Factorization of Hard Processes in QCD", in "Perturbative QCD", A. H. Mueller, ed., World Scientific, Singapore 1990

[9] G. T. Bodwin, Phys. Rev. D31, 2616 (1985);

G. T. Bodwin, S. J. Brodsky, G. P. Lepage: Phys. Rev. D39, 3287 (1989)

[10] A. H. Mueller, Les Houches Lectures 1991

[11] G. Sterman; "Factorization and Resummation", in "Perturbative and Nonperturbative Aspects of Quantum Field Theory", eds. H. Latal, W. Schweiger, to be published by Springer, Heidelberg

[12] J. Ellis, M. K. Gaillard, W. J. Zakrzewski: Phys. Lett. B81, 224 (1979)

[13] R. Doria, J. Frenkel, J. C. Taylor: Nucl. Phys. B168, 93 (1980)

[14] O. Nachtmann, A. Reiter: Z. Phys. C24, 283 (1984)

[15] T. T. Wu, C. N. Yang: Phys. Rev. B137, 708 (1965);

T. T. Chou, C. N. Yang: Phys. Rev. B170, 1591 (1968); Phys. Rev. D19, 3268 (1979); Phys. Lett. B128, 457 (1983); Phys. Lett. B244, 113 (1990);

J. Dias de Deus, P. Kroll: Phys. Lett. B60, 375 (1976); Nuovo Cimento A37, 67 (1977);

P. Kroll: Z. Phys. C15, 67 (1982);

J. Hüfner, B. Povh: Phys. Rev. D46, 990 (1992)

[16] C. Bourrely, J. Soffer, T. T. Wu: Nucl. Phys. B247, 15 (1984); Phys. Rev. Lett. 54, 757 (1985); Phys. Lett. B196, 237 (1987); Z. Phys. C37, 369 (1988);

R. J. Glauber, J. Velasco: Phys. Lett. B147, 380 (1984);

R. Henzi, P. Valin: Phys. Lett. B149, 239 (1984) 
[17] E. M. Levin, L. L. Frankfurt: JETP Lett. 2, 65 (1965);

H. J. Lipkin, F. Scheck: Phys. Rev. Lett. 16, 71 (1966);

H. J. Lipkin: Phys. Rev. Lett. 16, 1015 (1966);

J. J. J. Kokkedee, L. Van Hove: Nuovo Cimento A42, 711 (1966);

V. V. Anisovich et al.: "Quark Model and High Energy Collisions", World Scientific, Singapore 1985

[18] P. D. B. Collins: "An Introduction to Regge Theory", Cambridge University Press, Cambridge, U.K. 1977;

L. Caneschi, ed.: Regge Theory of low $p_{T}$ hadronic interaction, North Holland, Amsterdam 1989

[19] G. 't Hooft: Nucl. Phys. B72, 461 (1974);

G. Veneziano: Nucl. Phys. B74, 365 (1974); Phys. Lett. B52, 220 (1974); Nucl. Phys. B117, 519 (1976);

M. Ciafaloni, G. Marchesini, G. Veneziano: Nucl. Phys. B98, 472, 493 (1975);

A. Capella, U. Sukhatme, Chung-I Tan, J. Tran Thanh Van: Phys. Lett. B81, 68 (1979);

A. Capella, U. Sukhatme, J. Tran Thanh Van: Z. Phys. C3, 329 (1980); B. Andersson, G. Gustafson, G. Ingelman, T. Sjöstrand: Phys. Rep. C97, 31 (1983);

X. Artru: Phys. Rep. C97, 147 (1983);

B. Andersson, G. Gustafson, B. Nilsson-Almqvist: Nucl. Phys. B281, 289 (1987);

K. Werner: Phys. Rep. C232, 87 (1993)

[20] R. C. Hwa: Phys. Rev. D22, 759, 1593 (1980);

R. C. Hwa, M. Sajjad Zahir: Phys. Rev. D23, 2539 (1981);

R. C. Hwa: "Central production and small angle elastic scattering in the valon model", Proc. 12th Int. Symp. Multiparticle Dynamics, Notre Dame, 1981 (W. D. Shephard, V. P. Kenny, eds.) World Scientific, Singapore 1982

[21] E. A. Kuraev, L. N. Lipatov, V. S. Fadin: Sov. Phys. J.E.T.P. 44, 443 (1976), 45, 199 (1977);

L. N. Lipatov: Sov. J. Nucl. Phys. 23, 338 (1976);

Ya. Ya. Balitskii, L. N. Lipatov: Sov. J. Nucl. Phys. 28, 822 (1978); 
L. N. Lipatov: Sov. Phys. J.E.T.P. 63, 904 (1986);

L. N. Lipatov: "Pomeron in Quantum Chromodynamics", in "Perturbative Quantum Chromodynamics" (A. H. Mueller, Ed.), World Scientific, Singapore, 1989;

A. R. White: Int. J. Mod. Phys. A6, 1859 (1990);

J. Bartels: Z. Phys. C60, 471 (1993)

[22] F. E. Low: Phys. Rev. D12, 163 (1975);

S. Nussinov: Phys. Rev. Lett. 34, 1286 (1975);

J. F. Gunion, D. E. Soper: Phys. Rev. D15, 2617 (1977)

[23] A. Donnachie, P. V. Landshoff: Nucl. Phys. B244, 322 (1984); Nucl. Phys. B267, 690 (1986); Phys. Lett. B185, 403 (1987)

[24] W. Heisenberg: Z. Phys. 133, 65 (1952)

[25] H. Cheng, T. T. Wu: "Expanding Protons", MIT Press, Cambridge, Mass. 1987, and references cited therein.

[26] P. V. Landshoff, O. Nachtmann: Z. Phys. C35, 405 (1987)

[27] G. K. Savvidy: Phys. Lett. 71B, 133 (1977)

[28] A. I. Vainshtein, V. I. Zakharov, M. A. Shifman: JETP Lett. 27, 55 (1978)

[29] N.K. Nielsen, P. Olesen: Nucl. Phys. B144, 376 (1978)

[30] M. A. Shifman, A. I. Vainshtein, V. I. Zakharov: Nucl. Phys. B147, $385,448,519(1979)$

[31] G. 't Hooft: Cargèse Lectures, 1979, ed. G. 't Hooft et al., Plenum, New York, London 1980;

G. 't Hooft: Acta Phys. Austriaca, Suppl. 22, 531 (1980)

[32] G. Mack: Acta Phys. Austriaca, Suppl. 22, 509 (1980)

[33] J. Ambjørn, P. Olesen: Nucl. Phys. B170, 60, 265 (1980)

[34] H. Leutwyler: Phys. Lett. 96B, 154 (1980) 
[35] T. H. Hansson, K. Johnson, C. Peterson: Phys. Rev. D26, 2069 (1982)

[36] E. V. Shuryak: Phys. Rep. C115, 151 (1984), and references cited therein

[37] H. M. Fried, B. Müller (eds.): "QCD Vacuum Structure", World Scientific, Singapore 1993

[38] J. C. Maxwell: Philos. Mag. 21, 281 (1861), reproduced in: The Scientific Papers of James Clerk Maxwell, Vol. 1, p. 488; W. D. Niven, ed., Cambridge Univ. Press 1890

[39] H. G. Dosch: "Nonperturbative Methods in Quantum Chromodynamics", Progr. in Part. and Nucl. Phys. 33, 121 (1994)

[40] H. G. Dosch: Phys. Lett. B190, 177 (1987);

H. G. Dosch, Yu. A. Simonov: Phys. Lett. B205, 339 (1988);

Yu. A. Simonov: Nucl. Phys. B307, 512 (1988)

[41] J. Schwinger: Phys. Rev. 82, 664 (1951)

[42] I. Ya. Aref'eva: Theor. Mat. Phys. 43, 353 (1980);

N. E. Bralić: Phys. Rev. D22, 3090 (1980);

P. M. Fishbane, S. Gasioroviwcz, P. Kaus: Phys. Rev. D24, 2324 (1981);

L. Diosi: Phys. Rev. D27, 2552 (1983);

Yu. A. Simonov: Sov. J. Nucl. Phys. 48, 878 (1988)

[43] N. G. Van Kampen: Physica 74, 215, 239 (1974); Phys. Rep. C24, 172 (1976)

[44] A. DiGiacomo, H. Panagopoulos: Phys. Lett. B285, 133 (1992)

[45] H. G. Dosch, E. Ferreira, A. Krämer: Phys. Rev. D50, 1992 (1994)

[46] V. A. Novikow, M. A. Shifman, A. I. Vainshtein, V. I. Zakharov: Nucl. Phys. B191, 301 (1981)

[47] F. Borkowski et al., Nucl. Phys. A222, 269 (1974)

[48] F. Borkowski et al.: Nucl. Phys. B93, 461 (1975) 
[49] S. R. Amendolia et al.: Nucl. Phys. B277, 168 (1986)

[50] B. Povh, J. Hüfner, Phys. Rev. Lett. 58, 1612 (1987)

[51] E. Eichten, K. Gottfried, T. Kinoshita, K. D. Lane, T. M. Yan: Phys. Rev. D21, 203 (1980)

[52] M. Rueter, H. G. Dosch: Z. Phys. C66, 245 (1995)

[53] O. Nachtmann: Ann. Phys. 209, 436 (1991)

[54] B. Andersson, G. Gustafson, G. Ingelman, T. Sjöstrand: Phys. Rep. C97, 33 (1983)

[55] W. Buchmüller, A. Hebecker: "Semiclassical approach to structure functions at small $x "$ ", report DESY 95-208 (1995)

[56] H. Verlinde, E. Verlinde: "QCD at high energies and two-dimensional field theory" report PUPT-1319, IASSNS-HEP-92/30 (1993)

[57] L. Van Hove: Phys. Lett. 24B, 183 (1967)

[58] L. Lukaszuk, B. Nicolescu: Nuovo Cimento Lett. 8, 405 (1973);

D. Bernard, P. Gauron, B. Nicolescu: Phys. Lett. B199, 125 (1987);

E. Leader: Phys. Lett. B253, 457 (1991)

[59] A. Krämer, H. G. Dosch: Phys. Lett. B252, 669 (1990), B272, 114 (1991);

H. G. Dosch, E. Ferreira, A. Krämer: Phys. Lett. B289, 153 (1992)

[60] E. Berger, "Nichtstörungstheoretische Methoden zur Beschreibung von Reaktionen mit kleinem Impulstransfer in einem abelschen Modell", diploma thesis, Univ. of Heidelberg (1996), unpublished

[61] G. B. West: Phys. Lett. B115, 468 (1982);

C. D. Roberts, A. G. Williams: Progr. Part. and Nucl. Phys. 33, 477 (1994), and references cited therein;

K. Büttner, M. R. Pennington: "Infrared behaviour of the gluon propagator: Confining or confined?", Durham Univ. report DTP-95/32 (1995) 
[62] M. Rueter, H. G. Dosch: "Nucleon Structure and High Energy Scattering", Univ. of Heidelberg report HD-THEP-96-04, hep-ph/9603214 (1996)

[63] R. P. Feynman: Phys. Rev. Lett. 23, 1415 (1969); "Photon-Hadron Interactions", W. A. Benjamin, Reading, Mass. 1972

[64] I. M. Ternov, Yu. M. Loskutov, L. I. Korovina: Zh. Eskp. Teor. Fiz. 41, 1294 (1961) (Sov. Phys. - JETP 14, 921 (1962));

A. A. Sokolov, I. M. Ternov: Dokl. Akad. Nauk SSSR 153, 1052 (1963) (Sov. Phys.-Dokl. 8, 1203 (1964))

[65] J. D. Jackson: Rev. Mod. Phys. 48, 417 (1976)

[66] G. W. Botz, P. Haberl, O. Nachtmann: Z. Phys. C67, 143 (1995)

[67] F. E. Low: Phys. Rev. 110, 974 (1958)

[68] P. V. Chliapnikov et al.: Phys. Lett. 141B, 276 (1984)

[69] F. Botterweck et al.: Z. Phys. C51, 541 (1991)

[70] S. Abatzis et al.: Nucl. Phys. A525, 487c (1991)

[71] S. Banerjee et al. (SOPHIE/WA83 coll.): Phys. Lett. 305B, 182 (1993)

[72] J. Antos et al.: Z. Phys. C59, 547 (1993)

[73] E. Fermi: Z. Phys. 29, 315 (1924);

C. F. v. Weizsäcker: Z. Phys. 88, 612 (1934);

E. J. Williams: Kgl. Danske Videnskab. Selskab. Mat.-Fiz. Medd. 13, No 4 (1935)

[74] V. M. Budnev et al.: Phys. Rep. C15, 181 (1975)

[75] S. Platchkov et al.: Nucl. Phys. A510, 740 (1990)

[76] M. Meyerhoff et al.: "First measurement of the electric form factor of the neutron in the exclusive quasielastic scattering of polarized electrons from polarized ${ }^{3} H e$ ", Univ. Mainz Report (1994)

[77] H. Leeb, C. Teichtmeister: Phys. Rev. C48, 1719 (1993) 
[78] P. E. Bosted et al., Phys. Rev. Lett. 68, 3841 (1992)

[79] R. D. Carlitz, S. D. Ellis, R. Savit: Phys. Lett. 68B, 443 (1977);

N. Isgur, G. Karl, R. Koniuk: Phys. Rev. Lett. 41, 1269 (1978);

D. Gromes: "Ordinary Hadrons", in Proc. Yukon Adv. Study Inst.: The Quark Structure of Matter, eds. N. Isgur, G. Karl, P. J. O'Donnell (World Scientific, Singapore 1985)

[80] A. Brandenburg, E. Mirkes, O. Nachtmann: Z. Phys. C60, 697 (1993)

[81] S. Falciano et al., (NA10 coll.): Z. Phys. C31, 513 (1986);

M. Guanziroli et al., (NA10 coll.): Z. Phys. C37, 545 (1988)

[82] W. Buchmüller, A. Hebecker: Phys. Lett. B355, 573 (1995);

A. Edin, G. Ingelman, J. Rathsman: Phys. Lett. B366, 371 (1996)

[83] A. Efremov, D. Kharzeev: Phys. Lett. B366, 311 (1996)

[84] W. Bonivento et al. (DELPHI Coll.): "A measurement of quark spin correlations in hadronic $Z^{0}$ decays." Contribution to the EPS-HEP 95 conference, Brussels, August 1995

[85] H. B. Nielsen, A. Patkos: Nucl. Phys. B195, 137 (1982)

[86] S. V. Mikhailov, A. V. Radyushkin: Sov. J. Nucl. Phys. 49, 494 (1989)

[87] E. V. Shuryak: Nucl. Phys. B328, 85 (1989)

[88] P. Ball, V. M. Braun, H. G. Dosch: Phys. Rev. D44, 3567 (1991)

[89] C. Alexandrou, S. Güsken, F. Jegerlehner, K. Schilling, R. Sommer: Nucl. Phys. B414, 815 (1994);

R. Sommer: report DESY 94-011 (1994), unpublished

[90] U. M. Heller, K. M. Bitar, R. G. Edwards, A. D. Kennedy: Phys. Lett. B335, 71 (1994) 


\section{Figure Captions}

Fig. 1: The lowest order diagram for the Drell-Yan reaction (1.9) in the QCD improved parton model.

Fig. 2: The schematic behaviour of the vacuum energy density $\varepsilon(B)$ as function of a constant chromomagnetic field $B$ according to Savvidy's calculation (eq. (2.2)).

Fig. 3: A "snapshot" of the QCD vacuum showing a domain structure of spontaneously created chromomagnetic fields.

Fig. 4: The QCD-vacuum according to Ambjørn and Olesen 33 (a). The ether according to Maxwell [38] (b).

Fig. 5: Points $X, X^{\prime}, Y$ in Euclidean space time and curves $C_{X}, C_{X^{\prime}}$ running from $X$ to $Y$ and $X^{\prime}$ to $Y$, respectively.

Fig. 6: Curves in Euclidean space time: $C_{1}$ going from $X_{1}$ to $X_{2}, C_{2}$ from $X_{2}$ to $X_{3}, C$ from $X$ to $Y$, and $\bar{C}$ from $Y$ to $X$.

Fig. 7: A surface $S$ with boundary $C=\partial S$. A plaquette with corner points $Z_{1} \ldots, Z_{4}$ and boundary formed by lines $u=$ const, $v=$ const..

Fig. 8: A reference point $Y$ on $S$ and the fan-type net with center $Y$ spanned over $S$.

Fig. 9: The correlator function $4 \pi^{2} \kappa G_{2} D\left(-Z^{2}\right)$ (cf. (2.61)) as function of $|Z|$. The dashed line is the lattice result of [44] with the arrows indicating the range where these results are considered reliable. The solid line corresponds to the ansatz (2.65) with $a=0.35 \mathrm{fm}$ (cf. Fig. 5.1 of 450).

Fig. 10: Rectangular Wegner-Wilson loop in Euclidean space-time in the $X_{1}-X_{4}$ plane. The linear extensions are $R$ in $X_{1}$ direction and $T$ in $X_{4}$ direction. $Y$ is the reference point used in the application of the non-abelian Stokes theorem.

Fig. 11: The $t$-channel (a) and $u$-channel (b) exchange topologies for the diagrams describing quark-quark scattering. 
Fig. 12: Projection of the world lines of the quarks 1(2) moving at high velocity in positive (negative) $x^{3}$ direction onto the $x^{0}-x^{3}$ plane in Minkowski space. The non-abelian phase factors $V_{-}$in (3.35) $\left(V_{+}\right.$in (3.37)) are the connectors taken along lines $x_{-}, \mathbf{x}_{T}=$ const. $\left(x_{+}, \mathbf{x}_{T}=\right.$ const.).

Fig. 13: Two light-like lines on which the associated string operators $V_{ \pm}$in (3.45) are evaluated. Their correlation function governs quark-quark scattering at high energies.

Fig. 14: Sketch of reaction (3.50) in the overall c.m. system

Fig. 15: The light-like Wegner-Wilson loop in Minkowski space-time, $C_{+}$ consisting of two light-like lines in the hyperplane $x_{-}=0$ and connecting pieces at infinity. In transverse space the centre of the loop is at $\mathbf{y}_{T}$, the vector from the antiquark to the quark line is $\mathbf{z}_{T}$ (from $A$ to $B)$.

Fig. 16: The projections of the two lightlike Wegner-Wilson loops $C_{+}, C_{-}$ occurring in the definition of $W_{+}\left(\frac{1}{2} \mathbf{b}_{T}, \mathbf{x}_{T}\right), W_{-}\left(-\frac{1}{2} \mathbf{b}_{T}, \mathbf{y}_{T}\right)$ (cf. (3.87), (3.83) ) into transverse space. The points marked $q_{+}, \bar{q}_{+}\left(q_{-}, \bar{q}_{-}\right)$correspond to the projections of the quark and antiquark lines of $C_{+}\left(C_{-}\right)$.

Fig. 17: The curves $C_{+}$and $C_{-}$along which the path integrals $\mathcal{W}_{ \pm}$in (3.100) are taken. The mantle of the pyramid with apex at the origin of the coordinate system and boundary $C_{+}\left(C_{-}\right)$is $\mathcal{P}_{+}\left(\mathcal{P}_{-}\right)$, the basis surface $S_{+}\left(S_{-}\right)$.

Fig. 18: The relation between the total cross section $\sigma_{\text {tot }}$ and the slope parameter $b$ for proton-proton and proton-antiproton scattering. The dotted line is the prediction from Regge theory. The prediction of the calculation for soft high energy scattering in the stochastic vacuum model is that the data points should lie in the area between the full lines. In essence this is given by (3.108), (3.109) with an uncertainty estimate from different assumptions for the proton wave functions (cf. 45]).

Fig. 19: A proton-proton collision at high energies in the parton picture. 
Fig. 20: A quark and antiquark traversing a region of chromomagnetic field (a). An electron and a positron in a storage ring (b). In both cases we expect the emission of synchrotron radiation to occur.

Fig. 21: Sketch of a "colour domain" in Minkowski space and of the worldline of a quark from a fast hadron moving through it.

Fig. 22: A quark moving in 3-direction in a transverse (in 1-direction) chromomagnetic field of strength $g B_{c}$ and picking up a transverse momentum (in 2-direction) of magnitude $\bar{p}_{T}$ over a length $l_{\text {eff }}$. Here $\bar{p}_{T}$ is the mean transverse momentum of quarks in the hadron.

Fig. 23: The $\left|\mathbf{k}_{T}\right|$ distribution for direct photons emitted at c.m. rapidity $y=0$ in $p-B e$ collisions at $450 \mathrm{GeV}$ incident proton momentum from [72]. The normalization is according to a private communication by H. J. Specht. The background of decay photons is subtracted. The dash-dotted line gives the expected yield of photons from hadronic bremsstrahlung, the dashed lines show the upper and lower limits including the systematic errors in the shape of the decay background and the bremsstrahlung calculation (cf. [72]). The lower (upper) solid line is the result of the calculation for synchrotron photons (4.2ff.) with $l_{\text {eff }}=20 \mathrm{fm}\left(l_{\mathrm{eff}}=40 \mathrm{fm}\right)$ added to the spectrum of hadronic bremsstrahlung (cf. 66]).

Fig. 24: A nucleon interacting by emission of a quasi-real photon.

Fig. 25: The data for the electric form factor of the neutron $G_{E}^{n}\left(Q^{2}\right)$ from refs. [75, 76]. Dash-dotted line: our naive "synchrotron" prediction $\propto\left(Q^{2}\right)^{1 / 6}(4.13)$ normalized to the data at $Q^{2}=5 \mathrm{fm}^{-2}$. Dashed line: the slope of $G_{E}^{n}\left(Q^{2}\right)$ at $Q^{2}=0$ as deduced from thermal neutronelectron scattering [77]. Full line: the ansatz (4.15).

Fig. 26: The ratio $G_{E}^{p} / G_{D}$ of the electric fom factor of the proton to the dipole fit versus $Q^{2}$. The data points are from various experiments as summarized in [48]. The solid line corresponds to the ansatz (4.17), (4.18).

Fig. 27: Annihilation of a $q \bar{q}$ pair with production of a virtual photon $\gamma^{*}$ in a colour domain. Here $q$ and $\bar{q}$ come from two different hadrons $h_{1}$ and $h_{2}$, respectively. 
Fig. A1: The relevant integration region for the integral (A.8). The point (1) runs freely over the surface $S$, the point (2) is constrained to a distance $\lesssim a$ from (1).

Fig. A2: The relevant integration region for the integral (A.11). The point (1) runs freely over $S$. The points (1), (2), (3), (4) are ordered in the angle as seen from the reference point $Y$ due to the path ordering function $\Theta(1,2,3,4)$. The points (1), (3) and (2), (4) must be at a distance $\lesssim a$ to each other.

Fig. A3: The rectangle $S, C=\partial S$ and $N_{1}$ reference points $Y_{1}, \ldots, Y_{N_{1}}$ on the curve $C_{1}$. The area between $C_{1}$ and $C$ is partitioned in $N_{1}$ plaquettes $P_{1}, \ldots, P_{N_{1}}$.

Fig. A4: The potential $V(R)$ as defined in (A.39) with $R_{c}$ the "string breaking" radius. 
This figure "fig1-1.png" is available in "png" format from: http://arxiv.org/ps/hep-ph/9609365v1 
This figure "fig2-1.png" is available in "png" format from: http://arxiv.org/ps/hep-ph/9609365v1 
This figure "fig1-2.png" is available in "png" format from: http://arxiv.org/ps/hep-ph/9609365v1 
This figure "fig2-2.png" is available in "png" format from: http://arxiv.org/ps/hep-ph/9609365v1 
This figure "fig1-3.png" is available in "png" format from: http://arxiv.org/ps/hep-ph/9609365v1 